\title{
Vascular Consequences of Metabolic Syndrome Related Shifts in Cyclooxygenase Mediated Arachidonic Acid Metabolism
}

\author{
Adam G. Goodwill \\ West Virginia University
}

Follow this and additional works at: https://researchrepository.wvu.edu/etd

\section{Recommended Citation}

Goodwill, Adam G., "Vascular Consequences of Metabolic Syndrome Related Shifts in Cyclooxygenase Mediated Arachidonic Acid Metabolism" (2011). Graduate Theses, Dissertations, and Problem Reports. 3424.

https://researchrepository.wvu.edu/etd/3424

This Dissertation is protected by copyright and/or related rights. It has been brought to you by the The Research Repository @ WVU with permission from the rights-holder(s). You are free to use this Dissertation in any way that is permitted by the copyright and related rights legislation that applies to your use. For other uses you must obtain permission from the rights-holder(s) directly, unless additional rights are indicated by a Creative Commons license in the record and/ or on the work itself. This Dissertation has been accepted for inclusion in WVU Graduate Theses, Dissertations, and Problem Reports collection by an authorized administrator of The Research Repository @ WVU.

For more information, please contact researchrepository@mail.wvu.edu. 


\title{
Vascular Consequences of Metabolic Syndrome Related Shifts in Cyclooxygenase Mediated Arachidonic Acid Metabolism
}

\author{
Adam G. Goodwill \\ fulfillment of the requirements for the degree of \\ Doctor of Philosophy \\ In \\ Cellular and Integrative Physiology \\ Jefferson Frisbee Ph.D., Chair/Mentor \\ Julian Lombard Ph.D. External \\ Robert Brock Ph.D. \\ Alexandre d'Auddifret M.D. \\ Stan Hileman Ph.D. \\ John Hollander Ph.D. \\ Cellular and Integrative Physiology Program \\ Morgantown, West Virginia \\ 2011
}

Dissertation submitted to the School of Medicine at West Virginia University in partial

Key Words: metabolic syndrome, Zucker rat, rarefaction, cyclooxygenase, arachidonic acid 


\section{Abstract \\ Vascular Consequences of Metabolic Syndrome Related Shifts in Cyclooxygenase Mediated Arachidonic Acid Metabolism}

\section{Adam G. Goodwill}

The metabolic syndrome is a multi-faceted disease state in which the initial pathology of obesity leads to the development of a cadre of comorbidities including, but not limited to, atherogenic dyslipidemia, a prothrombotic state, a proinflammatory state, a pro-oxidant state, hypertension and type II diabetes mellitus. The prevalence of obesity is drastically increasing both nationally and globally, indicating that understanding obesity related disease is more relevant now then ever before. The metabolic syndrome has been demonstrated to result in numerous deleterious consequences for afflicted individuals, not the least of which is peripheral vascular disease. While the study of peripheral vascular disease has been extensively conducted and a robust body of literature exists implicating alterations in vasomotor regulation as the etiology for demand:perfusion mismatches in peripheral tissues, little study has been conducted to determine the consequences of metabolic syndrome on vascular network structure and the perfusion outcomes of the alterations to that network.

Our lab has previously demonstrated that with metabolic syndrome comes a progressive loss of microvessel density in skeletal muscle, termed rarefaction. This rarefactive phenomenon has been established in other animal models as well as in humans, however, the mechanistic underpinnings of microvascular rarefaction still require significant investigation. For this reason we set out to: 
1. Identify the significant physiologic correlates/predictors of microvascular rarefaction.

2. Determine which correlates played a contributing role in the development of rarefaction.

3. Better establish a time-course for the phenomenon of rarefaction in our experimental model; the obese Zucker rat.

The results of these studies demonstrate that alterations to inflammatory status and elevated oxidant stress significantly contribute to the development of obesity related rarefaction. Additionally, these two causative-agents act through intermediaries either shifting the balance in endogenous prostanoid production to a preferential generation of TXA2, or diminishing vascular nitric oxide bioavailability. Additionally, it was discovered that in the obese Zucker rat, rarefaction is a biphasic process wherein the phases are mechanistically distinct from one another. The initial or early phase of rarefaction is mediated by elevations in TxA2 production, whereas later rarefaction is mediated by diminished vascular nitric oxide bioavailability. Taken together, these data indicate that rarefaction is a process whose origins are rooted in the very earliest development of metabolic syndrome. The process itself is more mechanistically complex than initially anticipated and is mediated by pathologic alterations (elevated oxidant stress and altered inflammatory profile) which have been shown to produce additional deleterious effects. These studies would indicate that a greater knowledge of the origins and regulation of alterations to inflammatory status and elevations in oxidative stress are essential in treating metabolic syndrome. 


\section{Dedication}

To all the world's screw ups who were given a second chance and to my father who taught me that every morning, when you look in the mirror, you need to be ok with the quality of the man looking back at you. 


\section{Acknowledgements}

I hate the whole concept of an acknowledgements section. They are like Christmas cards. You set out with the best of intentions but, inevitably, you overlook someone and feelings are hurt. Therefore, to begin, I apologize to those who fail to be mentioned. It goes without saying that my graduate career and even my day-to-day life wouldn't be possible to maintain at the quality to which I have become accustomed without the help of countless family and friends. To those of you mentioned below and those whom I may fail to mention, I give my sincere thanks. I am fortunate to be surrounded by remarkable individuals and I am thankful for the time you give me each and every day.

To start out with I need to thank Fred Minnear Ph.D. Fred actually signed the letter that expelled me from this illustrious institution of higher learning. He also signed the letters reinstating me as a student. Without my expulsion, I would be an arrogant ass meandering my way through a lack-luster career. As it stands now, I am duly humbled and truly appreciative of the opportunities that have been presented to me and the doors that those opportunities have opened as I move forward. Fred, if you are reading this, you know that we have stood on the opposite sides of many issues. However, I have always admired that you stance on any issue is always determined by

your dedication to doing what you feel is right. What's right isn't always what's easy and I admire the principles that it takes to adhere to your course.

Special thanks also have to go out to the members of Frisbee laboratory, both past and present. Milinda, thank you for countless dialogues, endless assistance with 
various preparations, and for being a constant sounding board for ideas and problems. You have been a tremendous asset and a good friend. Phoebe, from you I received endless banter and a near paralyzing fear of saying anything that may be turned back against me. There was some science in there as well but I only vaguely remember it occurring between moments of sarcasm. Jordan (Beckett) Hartshorn, a sort of honorary Frisbee lab member, certainly deserves mention as well. I sincerely have no idea what inspires you to say the things that you say most of the time. You often render me speechless; a rare talent. You are certainly one of the most sincere individuals that I have ever met. I hope that quality survives all the training that you are currently undertaking. Finally, Josh (Joosh, Little Butchie) Butcher. Yep, it's in print now. These names are officially documented. You're welcome. To being with, thank you for all of the help with the experiments that gave me the final push to graduation. There was a lot of work and you bore it well. Remember that you are the immediate future of the lab. You have tremendous potential and, if you listen to Jeff, you'll go far. Keep in mind, most of the things that he says will make little to no sense at the time. Just roll with it. Jeff, I know that you are tired or reading this by now but we'll get to you in a bit...bear with me.

To my family, both immediate and extended, thank you. I can't imagine having to put up with me. You have all been supportive even when I had done nothing to earn that support. I never really say it, but I appreciate you more than you realize. I am blessed to have been born into a great family and to have married into another wonderful group. I hope that both my parents and my in-laws know how much I have 
valued what they have done to help me along the way and realize how much I love them all. Elizabeth, I even love and cherish you as well, $\sim 98 \%$ of the time.

My immediate family is an anomaly. I have a brilliant and beautiful wife and a daughter whose demeanor and intelligence bless her with near limitless potential. I am completely underserving of the both of you and I hope you never catch on to that fact. Thank you for smiles and tears. Thank you for understanding as I ignored you both these past few months to focus on my job. Thank you for just being a part of my life.

My immediate family also includes two fur-babies who bear mention. Duncan, you are a complete and total pain in the ass. You're my pain in the ass though and without you, I wouldn't really have any good stories to tell. Besides, every hero needs a nemesis. Bruenor, you epitomize dog. For 9 years, I have had a second shadow and many nights in the lab have only been made possible because I know you're there watching over my girls. I wish you that the two of you could read as this seems kind of stupid considering your illiteracy.

Of course, there is the Physiology Department and the Cardiovascular Center. You provided me with both an education and a professional family. Thank you for all that you have given me. Particular mention is needed for Vickie White who pretty much made sure that all of my screw-ups were fixed before they became a real issue. You also throw a mean tailgate. Thanks Victoria.

Finally we get to Jeff. Howdy boss. First of all, you made me cry at the beginning of my public defense. That was just in poor taste. I'll repay the favor someday. In Jefferson Frisbee I found a friend and mentor. Jeff was THE reason that 
my return to graduate school was possible. He was an advocate for me when that position was not a particularly popular one. He let me know when I was wrong, or when I was being a jerk but he also reminded me that if I focused, I could succeed. My entire first two years in the lab, I set out to prove that he was right. I wasn't doing it for me so much as I didn't want to let him down. At some point that changed and now the journey from here on out is my own. To Jeff, I owe a debt that I can never repay. He fundamentally changed who I am, I believe for the better.

With that I wrap up this little exposition on wonderful people. Thank you again to each of you as well as thanks to those who I will likely think of the very day that this dissertation gets submitted to the ETD folks. Now, without further ado, please feel free to read a bunch of the science that I did. You'll find it in the next couple of pages. 


\section{Abbreviations}

3-hydroxy-3-methylglutaryl coenzyme A

4-Hydroxy-2,2,6,6- tetramethylpiperidine- $-1-{ }^{15} \mathrm{~N}$-oxyl

6-keto-prostaglandin $\mathrm{F}_{1 \alpha}$

11-dehydro-thromboxane-B2

17-octadecynoic acid

Acetylcholine

Analysis of variance

Apolipoprotein E

Arachidonic Acid

Atorvastatin

Body Mass Index

Carboxyheptyl imidazole

Cyclooxygenase

Cytochrome P450

Endothelium Derived Relaxing Factors

Endothelial Nitric Oxide Synthase

Epoxyeicostreienoic Acid

Gemfibrozil

High Density Lipoprotein

Hydroxyeicosatetraenoic Acid

Indomethacin

Interleukin

$L-N^{G}$-nitroarginine methyl ester

Lean Zucker Rat

Lipoxygenase

Low Density Lipoprotein

Monocyte Chemotactic Protein-1

Nordihydroguaiaretic Acid

Nitric Oxide

Nitric Oxide Synthase

Obese Zucker Rat
(HMG Co-A)

(TEMPOL)

(6-keto-PGF ${ }_{1 \alpha}$ )

(11-dehydro-TxB ${ }_{2}$ )

(17-ODYA)

$(\mathrm{ACH})$

(ANOVA)

(ApoE)

(AA)

(ATOR)

(BMI)

$(\mathrm{CHI})$

(COX)

(CYP450)

(EDRF)

(eNOS)

(EETs)

(GEM)

(HDL)

(HETEs)

(INDO)

(IL)

(L-NAME)

(LZR)

(LOX)

(LDL)

(MCP-1)

(NDGA)

(NO)

(NOS)

(OZR) 
Polyethylene Glycol Superoxide Dismutase

Probucol

(PEG-SOD)

Prostacyclin

(PRO)

Prostaglandin F2 alpha

$\left(\mathrm{PGI}_{2}\right)$

Prostaglandin $\mathrm{H} 2$

(PGF2 $\alpha$ )

$\left(\mathrm{PGH}_{2}\right)$

Regulated Upon Activation Normal T-cell Expressed and Secreted (RANTES)

Simvastatin

(SIM)

Thromboxane A2

Tumor Necrosis Factor

$\left(\mathrm{TxA}_{2}\right)$

(TNF)

Vascular Endothelial Growth Factor

(VEGF) 


\section{TABLE OF CONTENTS}

Vascular Consequences of Metabolic Syndrome Related Shifts in Cyclooxygenase Mediated Arachidonic Acid Metabolism ......................................i

ABSTRACT DEDICATION

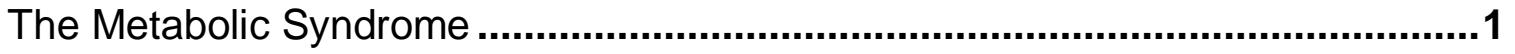

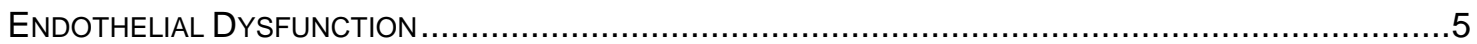

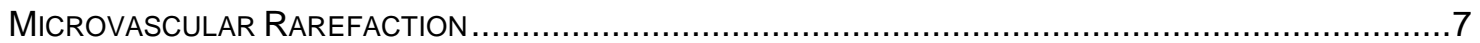

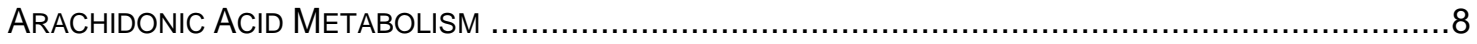

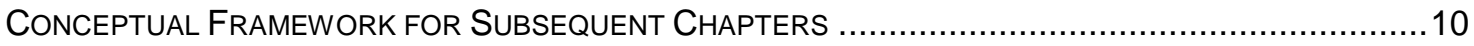

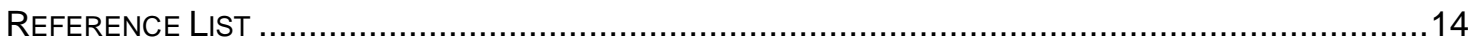

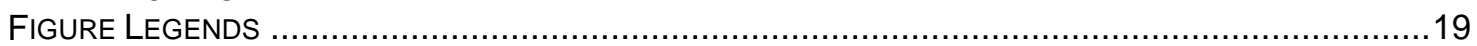

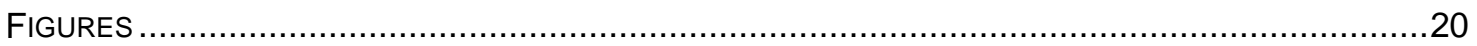

Increased Vascular Thromboxane Generation Impairs Dilation of Skeletal Muscle Arterioles of Obese Zucker Rats with Reduced Oxygen Tension ........................22

ABSTRACT INTRODUCTION

Increased Arachidonic Acid-Induced Thromboxane Generation Impairs Skeletal Muscle Arteriolar Dilation with Genetic Dyslipidemia ..........................................54

ABSTRACT 


\section{Impact of Chronic Anti-Cholesterol Therapy on Development of Microvascular}

Rarefaction in the Metabolic Syndrome ..........................................................89

ABSTRACT INTRODUCTION A

Temporal Progression of Microvascular Rarefaction in the Metabolic Syndrome; The Role of Thromboxane .........................................................................132

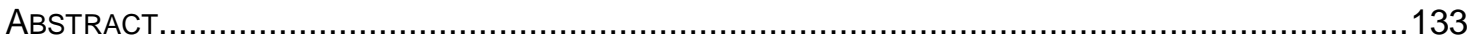

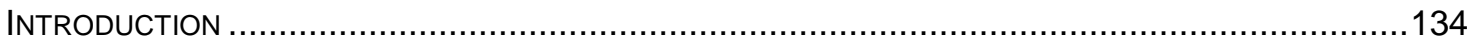

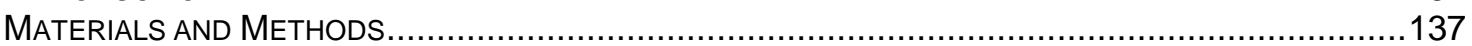

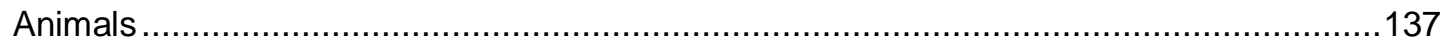

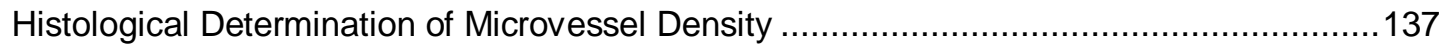

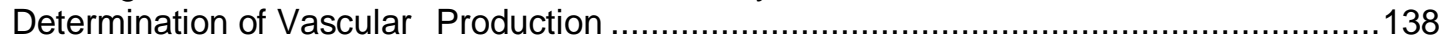

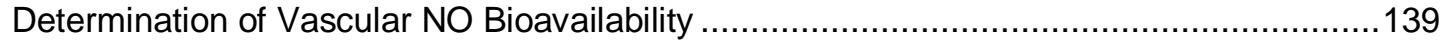

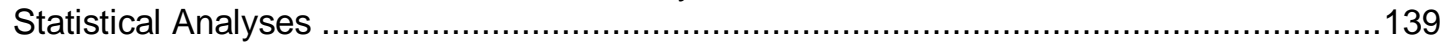

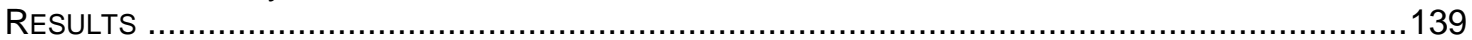

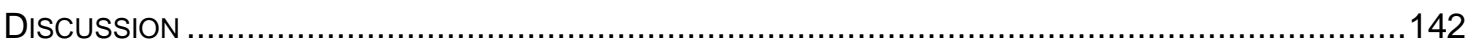

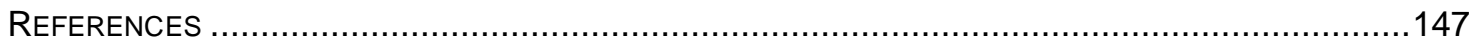

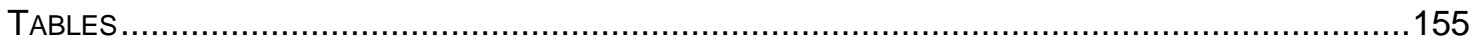

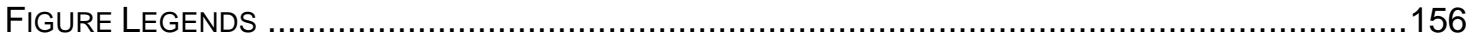

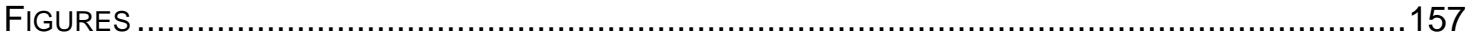

Discussion ............................................................................................... 161

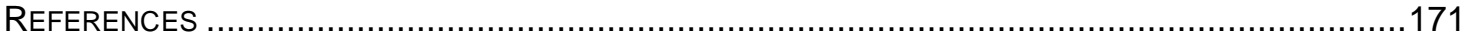

Permission to Reproduce Copyrighted Materials............................................173

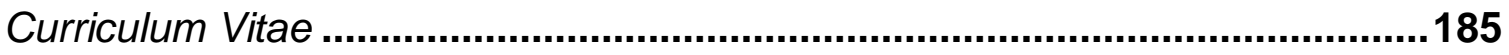


Chapter 1

The Metabolic Syndrome 
The metabolic syndrome is a term given to a constellation of comorbidities manifested within a single individual and owing to the initiating condition of obesity. The term itself has been in common, though poorly defined, usage as early as the 1950s. The first appearance of the term "metabolic syndrome" in a peer reviewed journal indexed by PUBMED was by an Israeli group in 1972 (11). Though they were the only group to publish a manuscript employing the term metabolic syndrome in that year, by 1977, the term began to be used annually in a limited number of publications and it was in the early 1990s that the study of metabolic syndrome became mainstream with publication numbers rising from two in 1990 to 113 in 1999 with the trend continuing to a startling 3734 publications containing the term metabolic syndrome in 2010 (See Figure 1).

This impressive growth in metabolic syndrome research directly parallels the growth in numbers of obese individuals here in the United States and abroad. According to the National Health and Nutrition Examination Surveys, approximately $33.8 \%$ of Americans are obese $(\mathrm{BMI}>30)$ with a nearly equivalent percentage meeting the criteria for overweight $(\mathrm{BMI}>25)(4)$. Sampling back to 1960 demonstrates a near exponential rise in the number of obese individuals starting in 1980 and continuing to the turn of the century (See Figure 2) (15). Fortunately it appears that this phenomenonal growth in obesity is beginning to plateau, though extensive speculation exists attributing the plateau to ever diminishing numbers of individuals whose BMI falls below 25 remaining to enter into overweight or obese categories. It is worth noting that while some regionality does exist with regards to the BMI of an average citizen, when examined by state it becomes apparent that there are no startlingly obese states 
causing a shift in the statistics as the current distribution ranges from $21 \%$ in Colorado to $34 \%$ in Mississippi (1). This trend in obesity shows no evidence of significant future abatement as approximately $17 \%$ of children age $2-19$ are already obese (14). It is in this overweight and obese population of children, adolescents and adults that you will find those individuals with the predisposing characteristics for development of metabolic syndrome and it is because of the high prevalence of obesity that approximately $34 \%$ of all adult Americans met the diagnostic criteria for metabolic syndrome in 2006 (3).

A problem that metabolic syndrome research has suffered for a number of years is an unsatisfactory definition for the diagnostic criteria of the syndrome itself. The combination in increase of incidence/prevalence of metabolic syndrome paired with the drastic increase in metabolic syndrome directed research efforts globally led the American Heart Association to set guidelines for the definition of metabolic syndrome in the National Cholesterol Education Program's Adult Treatment Panel III report in 2004 (9). In this panel, six key components of metabolic syndrome were identified: abdominal obesity, atherogenic dyslipidemia, hypertension, a proinflammatory state, a prothrombotic state and insulin resistance with or without glucose intolerance. It was further stated that abdominal obesity, clinically presented as increased waist circumference, was both the strongest predictor of metabolic syndrome as well as a key contributor to all other identified comorbidities (9). To meet the diagnostic criteria of metabolic syndrome, a single individual must present with a minimum of three of the above listed pathologies (9).

The metabolic syndrome can be thought of in lay-terms as an increase in the chance of a negative outcome subsequent to a cardiovascular event. This is due to the 
multifaceted complications that the metabolic syndrome presents to the vasculature itself (13). Highlighted vascular deficits included within the syndrome are: increased vascular tone, increased incidence of atheroma formation, increased incidence of thrombus formation, endothelial dysfunction, vascular wall remodeling, and vascular network remodeling $(6 ; 13)$. The outcome of this integrated vascular system dysfunction with the greatest impact on quality of life is peripheral vascular disease, wherein perfusion cannot be appropriately regulated to match changes in metabolic demand $(2 ; 5)$. This results in an obese individual who becomes obese as a result of an imbalance in caloric homeostasis and ultimately remains obese due to an inability in peripheral skeletal tissues to adequately supply blood flow to reverse this imbalance (5; 6). In other words, people go from being obese because of a lack of appropriate exercise to meet their caloric intake to being unable to increase their caloric consumption by skeletal muscle due to inadequate perfusion.

The work detailed within this document investigates the role of metabolic syndrome induced alterations in cyclooxygenase mediated arachidonic acid metabolism in skeletal muscle microvascular disease. Initial investigations will demonstrate the role of these alterations in vasomotor dysfunction in animal models of metabolic syndrome but which ultimately serve as the foundational work for the investigation into the role of this shift in the progressive loss of microvessel density (rarefaction) demonstrated within the syndrome. By the conclusion of this document, it is the author's intention to demonstrate a significant role for the shift in arachidonic metabolism in causing microvascular rarefaction and identify prospective, potentially clinically relevant targets for intervention meriting future investigation. 


\section{Endothelial Dysfunction}

Endothelial dysfunction is generically a loss of normal physiologic function in the vascular endothelium with regards to barrier, anti-thrombotic character, regulatory, vasomotor, etc $(18 ; 20 ; 23)$. Of particular relevance to the metabolic syndrome are the vasomotor consequences of endothelial dysfunction which can be defined as a diminished responsiveness of the vascular endothelium to vasodilator stimuli (primarily mediated through NO and prostacyclin; collectively termed endothelial derived relaxing factors) along with exaggerated responses to constrictor stimuli (6). This is a major cause of disparities in demand:perfusion matching in the peripheral tissues in metabolic syndrome subjects. In addition to being a hallmark of the metabolic syndrome, endothelial dysfunction is one of the most extensively studied vasculopathies today. Particular attention is paid to the role of endothelial nitric oxide synthase (eNOS) in the endothelial dysfunction literature.

As previously stated, endothelial dysfunction is defined, in part, by a diminished responsiveness of the vasculature to endothelium dependent vasodilator stimuli. The mechanisms by which these impairments can occur are varied and not mutually exclusive. They include scavenging of the endothelium derived relaxing factors (EDRF), diminished production of the EDRF, functional antagonism by a vasoconstrictive force, inability of the EDRF to reach the effector sites and decreased responsiveness of the vascular smooth muscle to the EDRF. Numerous studies have demonstrated that individuals and experimental animals with metabolic syndrome suffer from endothelial dysfunction $(5 ; 17)$. Many of these studies have further demonstrated the role of ROS in the etiology of endothelial dysfunction in metabolic syndrome 
subjects with additional studies demonstrating the therapeutic potential of antioxidants in treatment of the dysfunction $(21 ; 25)$ as well obesity associated acute myocardial infarctions (12). Since a major portion of this manuscript deals with the alterations in arachidonic acid metabolite balance, prostacyclin will be discussed in subsequent sections whereas a brief overview of eNOS and NO is detailed below.

The primary function of eNOS is the conversion of L-arginine to L-citrulline, resulting in the release of the potent vasodilator, nitric oxide (NO), as a reaction side product. While this role is well established, more recent studies of vascular pathologies have also had cause to focus on a phenomenon termed eNOS uncoupling. In eNOS uncoupling, limiting concentrations of either L-arginine or the cofactor tetrahydrobiopterin $\left(\mathrm{BH}_{4}\right)$ cause eNOS to demonstrate a NADPH oxidase-like activity resulting in the production of $\mathrm{O}_{2}^{-}(16)$. Depletion of $\mathrm{BH}_{4}$ does not necessarily stem from a metabolic inadequacy in all cases. Studies have demonstrated that peroxynitrite can oxidize the essential cofactor for eNOS activity, tetrahydrobiopterin $\left(\mathrm{BH}_{4}\right)$ thereby uncoupling eNOS (24). In a sense this is a feedback loop as peroxynitrite is the product of the interaction between superoxide and NO. Therefore, with a modest increase in global oxidant stress comes the potential for a positive feedback loop wherein a small production of peroxynitrite can in turn scavenge $\mathrm{BH} 4$, thereby uncoupling eNOS resulting in the production of superoxide which could in turn interact to produce additional peroxynitrite and so on. This serves as a mechanism by which global oxidant stress can not only increase ROS production through secondary pathways but also attenuate the production of normal vaso-regulatory compounds. 
Additionally, since peroxynitrite is generated by the interaction of $\mathrm{O}_{2}^{-}$with $\mathrm{NO}$, as previously discussed, the effect on the systemic vasculature is exaggerated by both scavenging the existing NO and altering the catalytic activity of eNOS blocking further NO production. Peroxynitrite is not, however, alone in its ability to crosstalk and uncouple eNOS. Angiostatin, high glucose, ceramide, homocysteine and hypochlorous acid have all been shown to promote uncoupling in cultured endothelial cells.

\section{Microvascular Rarefaction}

Generically, rarefaction is a term defining any process wherein your subject or target of interest becomes less prevalent. In the case of microvascular rarefaction, this means that the arterioles and capillaries within a tissue of interest are becoming less prevalent when compared between conditions. The metabolic syndrome, demonstrates the process of microvessel rarefaction in skeletal muscle tissues when comparing between comparable lean and obese animals or humans. The consequence of this rarefactive process is the inability to match tissue demand and perfusion even in maximally dilated vascular beds. This was clearly demonstrated in 2003 in work by Frisbee et al. showing that the consequence of significant rarefaction is a true structural inability to match demand and perfusion resulting in a disparity with no short term temporal solution.

While the fact of rarefaction is established, the mechanisms of rarefaction have classically been poorly understood. In metabolic syndrome, rarefaction has been attributed to hypertension, dyslipidemia, insulin sensitivity, and vascular NO bioavailability. Using a technique first pioneered by Greene et al., (8) we are able to 
examine skeletal muscle microvessel density in animal models of metabolic syndrome rapidly and with high resolution. In this document, we will demonstrate that the process of rarefaction is a multi-mechanistic continuum of problems resulting in diminished tissue capillarity in the skeletal muscle of metabolic syndrome animal models.

\section{Arachidonic Acid Metabolism}

Arachidonic acid (AA) is stored in the membranes of nearly all known mammalian cells bound to membrane phospholipids. It can be released from this stored state by the actions of phospholipase A, phospholipase C or phospholipase D. As phospholipase A is capable of releasing AA in a single step as opposed to the multistep processes of phospholipases $C \& D$, it is generally the focus of the most research attentions. Subsequent to phospholipase mediated release from the plasma membrane, free vascular AA has 3 basic fates. It can be reincorporated into the plasma membrane bound once again to membrane phospholipids, it can diffuse away in the bloodstream to distal locations or it can diffuse into the cytoplasmic space of the cell. If AA diffuses into the cytoplasm, it is enzymatically degraded down one of three major enzymatic pathways: the cyclooxygenase pathway (COX), the lipoxygenase pathway (LOX) or the cytochrome P450 pathway (CYP450).

The cyclooxygenase pathway is the major enzymatic producer of a class of compounds known as prostanoids. These metabolic end-products of AA metabolism are the result of the activity of cyclooxygenase converting AA into prostaglandin $\mathrm{H} 2$. Prostaglandin $\mathrm{H} 2$ itself serves as the precursor to all series 2 prostanoids including prostacyclins, thromboxanes and prostaglandins. While all 3 categories of series 2 
prostanoids have been shown to produce numerous biological effects, prostacyclin and thromboxane generally receive the greatest attention in the cardiovascular literature as there is extensive evidence indicating both classes of compounds are key regulators of vasomotor tone in specific physiologic and pathophysiologic conditions. Classically these vascular considerations of thromboxane and prostacyclin are limited to the vasomotor as they are a potent vasoconstrictor and vasodilator, respectively.

Alternative to the cyclooxygenase pathway is the $5,12,15$ lipoxygenase pathway. This family of enzymes is responsible for catalyzing the conversion of arachidonic acid into a family of compounds collectively referred to as the leukotrienes. Leukotrienes are not considered to be major regulators of vascular tone as their effects are more relevant in the mediation of immune responses. Leukotriene A4 in particular has been implicated strongly in allergic asthma though its effects are not unique to that condition. Neutrophil recruitment, direct tracheal smooth muscle contraction, sustainment of inflammatory responses and acute allergic actions have all been attributed to leukotriene dependent processes.

The final pathway for the enzymatic metabolism of arachidonic acid is the CYP 450 pathway. This pathway can be broken into two subcategories: the epoxygenase pathway and the $\omega$-hydroxylase pathway. While both pathways are controlled by the activity of p-type-cytochromes and act on the same precursor, the epoxygenase pathway generates a class of compounds known as epoxyeicostreienoic acids (EETs) while the $\omega$-hydroxylase pathway is responsible for the production of hydroxyeicosatetraenoic acid (HETES). While roles for both classes of compounds have been indicated in the regulation of vascular tone, as well as roles in inflammation 
and oxidative stress, the studies contained herein failed to establish a role for CYP450 metabolites of AA and as such further discussion of these pathways will not be included.

Alterations in AA metabolism can have direct effects on vasomotor function. Increases in vascular oxidant stress can lead to shifts in the metabolism of AA through the COX pathway independent of the direct effects of ROS on prostacyclin synthase. Studies performed in the obese Zucker rat demonstrate that with changes to vascular oxidant stress, comes a shift in the metabolism of arachidonic acid through the COX pathway from a predominant production of prostacyclin to a predominant production of thromboxane $\left(\mathrm{TXA}_{2}\right)$ in response to hypoxia (7). The net effect of this shift is a blunted vasodilation in response to a highly specific vasodilator stimulus. This finding has been supported by additional studies which have demonstrated increased production of the vasoconstrictors $\mathrm{PGH}_{2}$ (19), $\mathrm{PGF}_{2 \alpha}$ (22) and $\mathrm{TXA}_{2}$ (10) along with diminished prostacyclin production in the diabetic experimental animals. This shift in arachidonic acid metabolism to the generation of greater production of vasoconstrictor stimuli serves to blunt the response of the endothelium to vasodilator stimuli as the vasodilator functions are masked by the tonic vasoconstrictive forces. While these effects have been documented within the literature, this manuscript will set out to establish a role for alterations in AA metabolism which stretch beyond the vasomotor and show demonstrable, significant, deleterious effects on vascular network structure.

\section{Conceptual Framework for Subsequent Chapters}

Due to the fact that the majority of remaining chapters in this document were originally intended as stand-alone texts, the logic linking each chapter to its predecessor 
and successor may not be immediately apparent. As such, we present here a conceptual framework in which to consider each manuscript contained within this body of work as a part of a greater intellectual approach to the study of vascular consequences of metabolic syndrome and the roles of arachidonic acid metabolites in these pathophysiologic processes. Chapter 2 of this document is entitled "Increased Vascular Thromboxane Generation Impairs Dilation of Skeletal Muscle Arterioles of Obese Zucker Rats with Reduced Oxygen Tension." This chapter is the foundational work upon which the author has continued his experimental designs through the course of his graduate education. It makes the initial observation that when a highly specific stimulus for vascular prostacyclin production, hypoxia, is employed in both lean and obese Zucker rats, there is a blunted dilation with obesity that is mediated exclusively by a shift from a predominant production of prostacyclin to a blunted prostacyclin and exaggerated thromboxane generation response. Additionally, this chapter provides some of the initial experiments indicating a role for elevations in oxidant stress as mediators for elevations in thromboxane generation.

Chapter 3 of this document is entitled "Increased Arachidonic Acid-Induced Thromboxane Generation Impairs Skeletal Muscle Arteriolar Dilation with Genetic Dyslipidemia. In this chapter, we leave the obese Zucker rat model and instead focus on two genetic variants of the $\mathrm{C} 57 / \mathrm{BI} / 6 \mathrm{~J}$ mouse with specific gene deletions of either the low density lipoprotein receptor or the apolipoprotein $\mathrm{E}$ gene resulting in an animal model of familial hypercholesterolemia or type III hyperlipidemia respectively. In this study we demonstrate that in an animal model of hypercholesterolemia, a comorobidity of metabolic syndrome, AA metabolism shifts in a directionally consistent manner with 
the more complex disease state of metabolic syndrome. Additionally, this manuscript provides further evidence for elevations in ROS as a mediator of the shift in AA metabolites.

Chapter 4 of this manuscript is titled "Impact of Chronic Anticholesterol Therapy on Development of Microvascular Rarefaction in the Metabolic Syndrome." This chapter returns to the obese Zucker rat model investigating how statin and fibrate therapies affect progression of skeletal muscle microvascular rarefaction in the metabolic syndrome. This paper, interestingly, demonstrates that protective effects of statin therapies can be conferred with regards to rarefaction, however, these effects are independent of plasma cholesterol levels and seem to be mediated by the pleiotropic effects in diminishing oxidative stress and inflammatory status. This paper serves a critical role in further demonstrating that microvascular rarefaction in fully developed metabolic syndrome animal models strongly correlates with diminished vascular NO bioavailability and that protection against microvessel loss is only conferred by those therapeutics capable of protecting against the loss of NO bioavailability.

The culmination of these ongoing investigations into metabolic syndrome related microvascular rarefaction is presented in Chapter 5 entitled, "Temporal Progression of Microvascular Rarefaction in the Metabolic Syndrome; The Role of Thromboxane A2." This incomplete work shows the preliminary investigations into a higher resolution, temporal analysis of the process of rarefaction in metabolic syndrome. It clearly demonstrates a biphasic rarefactive process mediated in the early phase by elevations in thromboxane A2 levels and in the later phase by diminished vascular NO bioavailability. This chapter provides the evidence that the early alterations to COX 
mediated AA metabolism have effects that extend beyond the vasomotor with significant impact on vascular network structure through the life of the animal. Supplementary materials which may provide a greater appreciation for the work presented within this manuscript include "Differential Impact of Familial Hypercholesterolemia and Combined Hyperlipidemia on Vascular Wall and Network Remodeling in Mice" by Stapleton et al. which provides some of the foundational correlative data between elevation in TxA2 production and changes to vascular wall mechanics in hyperlipidemic mice. Additional insight into the significance of this work can be found in the article entitled "Aspirin Resistance with Genetic Dyslipidemia: Contribution of Vascular Thromboxane Generation" by Frisbee et al. which was also performed in dyslipidemic mice and highlights why inhibition of COX is a poor therapeutic target in an animal model with a single metabolic syndrome comorbidity and helps to better explain why our investigations into metabolic syndrome related skeletal muscle microvascular rarefaction employ more selective inhibition of enzymes downstream from COX as opposed to general COX inhibition which may be more clinically employed though, plausibly, less therapeutically relevant. 


\section{Reference List}

1. CDC Fact Sheet. U.S. Obesity Trends: Trends by State 1985-2010 [Online]. 2011.

2. Costa LA, Canani LH, Lisboa HR, Tres GS and Gross JL. Aggregation of features of the metabolic syndrome is associated with increased prevalence of chronic complications in Type 2 diabetes. Diabet Med 21: 252-255, 2004.

3. Ervin RB. Prevalence of metabolic syndrome among adults 20 years of age and over, by sex, age, race and ethnicity, and body mass index: United States, 20032006. Natl Health Stat Report 1-7, 2009.

4. Flegal KM, Carroll MD, Ogden CL and Curtin LR. Prevalence and trends in obesity among US adults, 1999-2008. JAMA 303: 235-241, 2010.

5. Frisbee JC, Goodwill AG, Butcher JT and Olfert IM. Divergence between arterial perfusion and fatigue resistance in skeletal muscle in the metabolic syndrome. Exp Physiol 96: 369-383, 2011.

6. Frisbee JC, Wu F, Goodwill AG, Butcher JT and Beard DA. Spatial Heterogeneity in Skeletal Muscle Microvascular Blood Flow Distribution is Increased in the Metabolic Syndrome. Am J Physiol Regul Integr Comp Physiol 2011. 
7. Goodwill AG, James ME and Frisbee JC. Increased vascular thromboxane generation impairs dilation of skeletal muscle arterioles of obese Zucker rats with reduced oxygen tension. Am J Physiol Heart Circ Physiol 295: H1522-H1528, 2008.

8. Greene AS and Rieder MJ. Measurement of vascular density. Methods Mol Med 51: 489-496, 2001

9. Grundy SM, Brewer HB, Jr., Cleeman JI, Smith SC, Jr. and Lenfant C. Definition of metabolic syndrome: Report of the National Heart, Lung, and Blood Institute/American Heart Association conference on scientific issues related to definition. Circulation 109: 433-438, 2004.

10. Harrison HE, Reece AH and Johnson M. Decreased vascular prostacyclin in experimental diabetes. Life Sci 23: 351-355, 1978.

11. Herberg L, Bergmann M, Hennigs U, Major E and Gries FA. Influence of diet on the metabolic syndrome of obesity. Isr J Med Sci 8: 822-823, 1972.

12. Jaxa-Chamiec T, Bednarz B, Drozdowska D, Gessek J, Gniot J, Janik K, Kawka-Urbanek T, Maciejewski P, Ogorek M and Szpajer M. Antioxidant effects of combined vitamins $\mathrm{C}$ and $\mathrm{E}$ in acute myocardial infarction. The randomized, double-blind, placebo controlled, multicenter pilot Myocardial Infarction and VITamins (MIVIT) trial. Kardiol Pol 62: 344-350, 2005. 
13. Keller KB and Lemberg L. Obesity and the metabolic syndrome. Am J Crit Care 12: 167-170, 2003.

14. Ogden CL and Carroll MD.2010 Prevalence of Obesity Among Children and Adolescents: United States, Trends 1963-1965 Through 2007-2008 [Online]. 2010.

15. Ogden CL and Carroll MD. Prevalence of Overweight, Obesity, and Extreme Obesity Among Adults: United States, Trends 1960-1962 Through 2007-2008 [Online]. 2010.

16. Ramana KV, Chandra D, Srivastava S, Bhatnagar A and Srivastava SK. Nitric oxide regulates the polyol pathway of glucose metabolism in vascular smooth muscle cells. FASEB J 17: 417-425, 2003.

17. Rana JS, Jansen AC, Zwinderman AH, Nieuwdorp M, van Aalst-Cohen ES, Jukema JW, Trip MD and Kastelein JJ. Metabolic syndrome and risk of coronary, cerebral, and peripheral vascular disease in a large Dutch population with familial hypercholesterolemia. Diabetes Care 29: 1125-1127, 2006.

18. Santilli F, Vazzana N, Liani R, Guagnano MT and Davi G. Platelet activation in obesity and metabolic syndrome. Obes Rev 2011. 
19. Shimizu K, Muramatsu M, Kakegawa Y, Asano H, Toki Y, Miyazaki Y, Okumura K, Hashimoto $\mathbf{H}$ and Ito $\mathbf{T}$. Role of prostaglandin $\mathrm{H} 2$ as an endothelium-derived contracting factor in diabetic state. Diabetes 42: 1246-1252, 1993.

20. Siervo M, Jackson SJ and Bluck LJ. In-vivo nitric oxide synthesis is reduced in obese patients with metabolic syndrome: application of a novel stable isotopic method. J Hypertens 29: 1515-1527, 2011.

21. Skyrme-Jones RA, O'Brien RC, Berry KL and Meredith IT. Vitamin E supplementation improves endothelial function in type I diabetes mellitus: a randomized, placebo-controlled study. J Am Coll Cardiol 36: 94-102, 2000.

22. Subbiah MT and Dietemeyer D. Altered synthesis of prostaglandins in platelet and aorta from spontaneously diabetic Wistar rats. Biochem Med 23: 231-235, 1980.

23. Tsuji S and Node K. [Vascular endothelial dysfunction as a mechanistic factor for metabolic syndrome]. Nihon Rinsho 69 Suppl 1: 295-300, 2011.

24. Vlassara $\mathbf{H}$. The AGE-receptor in the pathogenesis of diabetic complications. Diabetes Metab Res Rev 17: 436-443, 2001. 
25. Zhang $\mathbf{H}$, Zhang J, Ungvari Z and Zhang $\mathbf{C}$. Resveratrol improves endothelial function: role of TNF\{alpha\} and vascular oxidative stress. Arterioscler Thromb Vasc Biol 29: 1164-1171, 2009. 


\section{FIGURE LEGENDS}

Figure 1. This figure legend is directly taken, as is the figure from the associated citation: Age adjusted by the direct method to the year 2000 U.S. Census Bureau estimates, using the age groups 20-39, 40-59, and 60-74 years. Pregnant females were excluded. Overweight is defined as a body mass index (BMI) of 25 or greater but less than 30; obesity is a BMI greater than or equal to 30 ; extreme obesity is a BMI greater than or equal to 40. Source: CDC/NCHS, National Health Examination Survey cycle 1 (1960-1962); National Health and Nutrition Examination Survey (1971-1974), II (1976-1980), and III (1988-1994), 1999-2000, 2001-2002, 2003-2004, 2005-2006, and 2007-2008. (15)

Figure 2. Annual usage of the term "metabolic syndrome" as determined by an independent search of PUBMED. 
Figure 1.

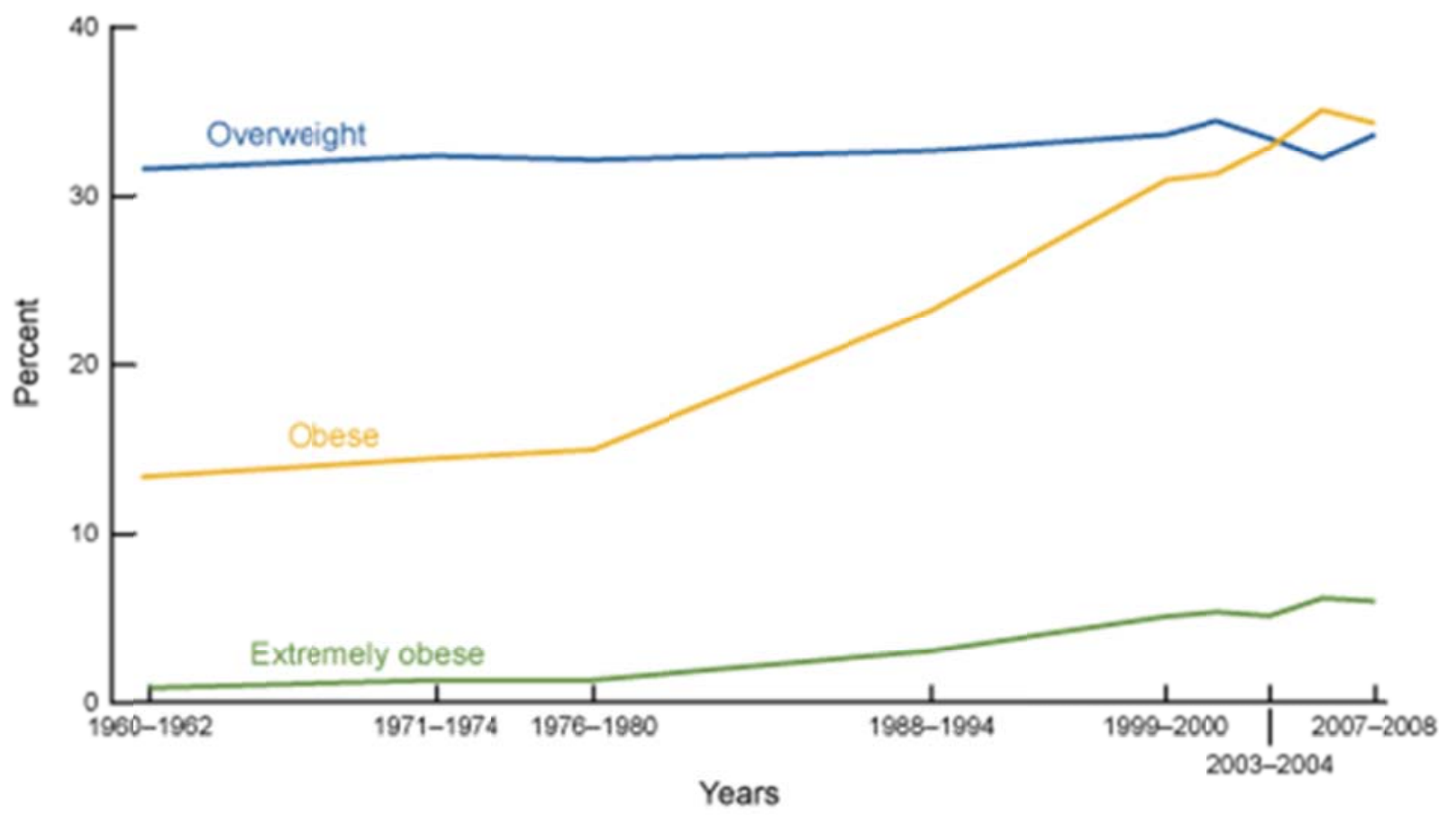

CDC/NCHS, National Health Examination Survey cycle 1 (1960-1962); National Health and Nutrition Examination Survey (1971-1974), II (1976-1980), and III (1988-1994), 1999-2000, 2001-2002, 2003-2004, 2005-2006, and 2007-2008. (15) 
Figure 2.

Metabolic Syndrome Publications

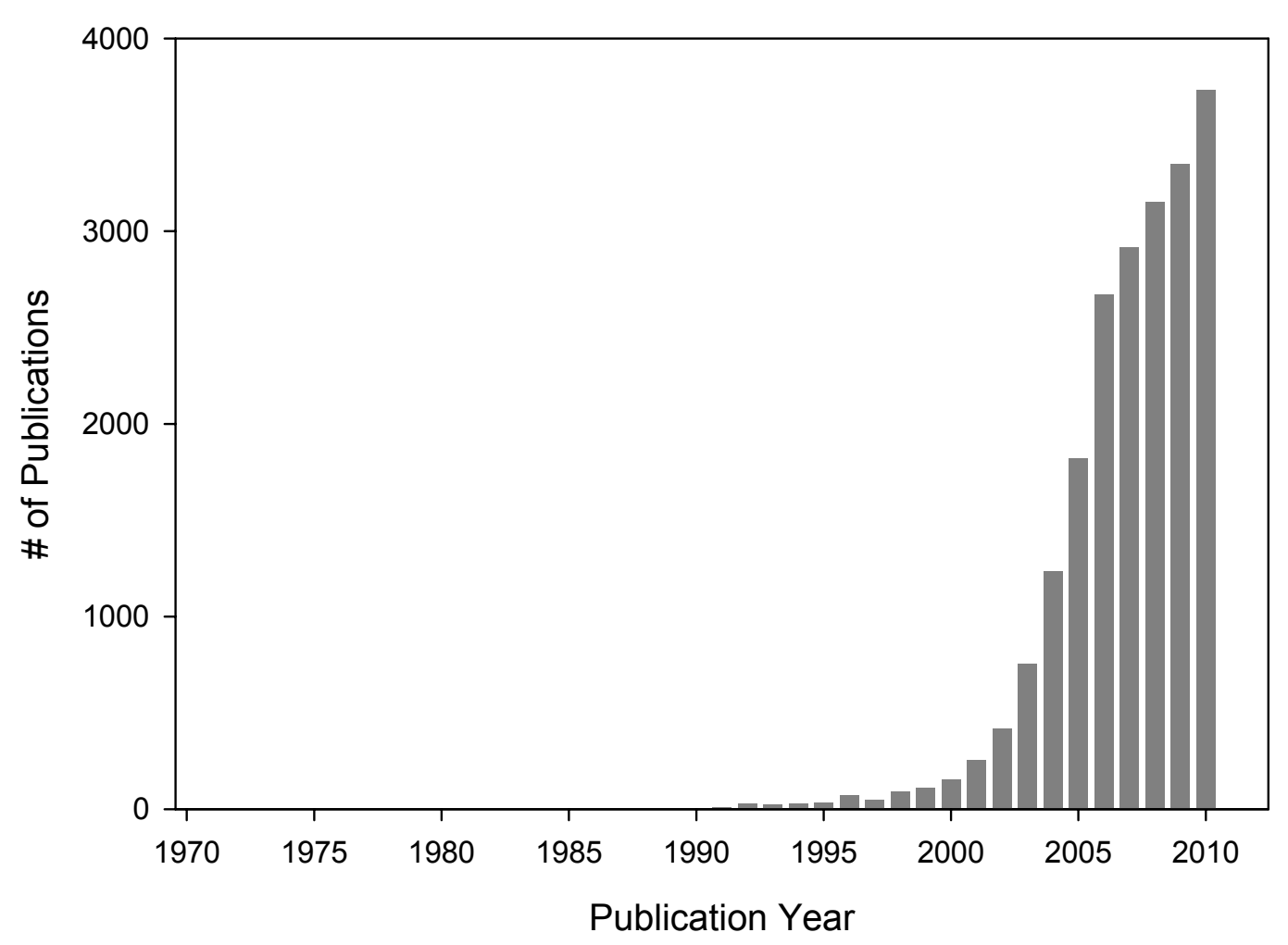




\title{
CHAPTER 2
}

\section{INCREASED VASCULAR THROMBOXANE GENERATION IMPAIRS DILATION OF SKELETAL MUSCLE ARTERIOLES OF OBESE ZUCKER RATS WITH REDUCED OXYGEN TENSION}

\author{
Adam G. Goodwill, Milinda E. James and Jefferson C. Frisbee \\ Center for Interdisciplinary Research in Cardiovascular Sciences \\ Department of Physiology and Pharmacology \\ West Virginia University School of Medicine, Morgantown, WV 26506
}

Running Head: Vasodilation and metabolic syndrome

"This research was originally published in The American Journal of Physiology Heart \& Circulatory Physiology. Goodwill AG, James ME, Frisbee JC . Increased vascular thromboxane generation impairs dilation of skeletal muscle arterioles of obese Zucker rats with reduced oxygen tension. Am J Physiol Heart Circ Physiol. 2008 Oct;295(4):H1522-8. “ 


\section{ABSTRACT}

This study determined if altered vascular prostacyclin $\left(\mathrm{PGI}_{2}\right)$ and/or thromboxane $\left(\mathrm{TxA}_{2}\right)$ production with reduced $\mathrm{PO}_{2}$ contributes to impaired hypoxic dilation of skeletal muscle resistance arterioles of obese Zucker rats (OZR) vs. lean Zucker rats (LZR). Mechanical responses were assessed in isolated gracilis muscle arterioles following reductions in $\mathrm{PO}_{2}$ under control conditions and following pharmacological interventions inhibiting arachidonic acid metabolism, nitric oxide synthase, and alleviating elevated vascular oxidant stress. Production of arachidonic acid metabolites was assessed using pooled arteries from OZR and LZR in response to reduced $\mathrm{PO}_{2}$. Hypoxic dilation, endothelium-dependent in both strains, was attenuated in OZR vs. LZR. NOS inhibition had no significant impact on hypoxic dilation in either strain. COX inhibition dramatically reduced hypoxic dilation in LZR and abolished responses in OZR. Treating arterioles from OZR with PEG-SOD improved hypoxic dilation, and this improvement was entirely COX-dependent. Vascular $\mathrm{PGI}_{2}$ production with reduced oxygen tension was similar between strains, although $\mathrm{TxA}_{2}$ production was increased in OZR; a difference that was attenuated by treatment of vessels from OZR with PEG-SOD. Both blockade of $\mathrm{PGH}_{2} / \mathrm{TxA}_{2}$ receptors and inhibition of thromboxane synthase increased hypoxic dilation in OZR arterioles. These results suggest that a contributing mechanism underlying impaired hypoxic dilation of skeletal muscle arterioles of OZR may be an increased vascular production of $\mathrm{TxA}_{2}$, which competes against the vasodilator influences of $\mathrm{PGI}_{2}$.

These results also suggest that the elevated vascular oxidant stress inherent in the metabolic syndrome may contribute to the increased vascular $\mathrm{TxA}_{2}$ production and may blunt vascular sensitivity to $\mathrm{PGI}_{2}$. 
Key Words: skeletal muscle microcirculation, endothelium-dependent dilation, vascular reactivity, rodent models of obesity 


\section{INTRODUCTION}

The metabolic syndrome represents a series of systemic pathologies that develop sequentially in afflicted individuals, and can include obesity, insulin resistance/type II diabetes mellitus, dyslipidemia and hypertension (25). While each of these pathologies in isolation can increase the future risk for development of peripheral vascular disease, when present in combination this risk increases dramatically (24), and can lead to numerous profound alterations to vascular structure/function relationships $(26,27)$. These vascular alterations can impair to tissue perfusion:demand matching and can lead to a compromised function (27). An effective animal model for the metabolic syndrome in humans is the obese Zucker rat (OZR), a rodent model characterized by its dysfunctional leptin receptor gene, resulting in abrogated leptin signaling and an impaired satiety reflex (10). As a result, the OZR experiences a chronic hyperphagia, and sequentially develops each of the systemic pathologies listed above, in addition to both pro-oxidant and proinflammatory states $(1,6,22)$. Previous studies by multiple investigative groups have demonstrated negative vascular outcomes in OZR with development of the metabolic syndrome, including alterations to vascular wall mechanics $(4,13,28,29)$, impairments to multiple indices of dilator reactivity $(9,13,15,16,33)$, signaling mechanisms underlying constrictor reactivity $(17,21,30)$ and a rarefaction of microvascular networks within multiple tissues $(7,8,31)$. The culmination of these alterations to microvascular structure and function within OZR can result in profound impairments to the perfusion of tissue under an array of physiological and pathological conditions $(8,12,33)$.

We have previously demonstrated that the dilator reactivity of skeletal muscle resistance arterioles in response to an acute reduction in oxygen tension is impaired in 
OZR compared to LZR, and that a contributing mechanism to this impairment lies within the chronic elevation in vascular oxidant stress (9). However, while this previous study did not implicate specific mechanisms beyond the contribution of elevated vascular oxidant stress, recent studies from Hester's group have provided data which may have considerable bearing on not only our previous observation, but also on the integrated regulation of vascular reactivity in the skeletal muscle of $\operatorname{OZR}(32,33)$. Specifically, the dilator response of in situ spinotrapezius muscle arterioles with increased metabolic demand, previously determined to be a largely dependent on vascular production of prostacyclin ( $\mathrm{PGI}_{2}$, Ref. 11), was blunted in OZR compared to LZR. This observation may reflect an enhanced vascular production of thromboxane $A_{2}\left(\mathrm{TxA}_{2}\right)$, or elevated vasoconstrictor response to $\mathrm{TxA}_{2}$ within OZR that is not present in lean animals $(32,33)$. Clearly, any increase in $\mathrm{TxA}_{2}$ production has the potential to compete against $\mathrm{PGI}_{2^{-}}$ mediated responses and impair dilator reactivity to multiple vasoactive stimuli.

The purpose of the present study was to more fully elucidate mechanisms contributing to the impaired dilation of skeletal muscle arterioles of OZR in response to reduced $\mathrm{PO}_{2}$. Additionally, the present study was designed to more clearly determine the role for elevated vascular oxidant stress in contributing to the attenuated hypoxic dilation of arterioles from OZR. Specifically, the hypothesis tested by the present experiments was that the compromised dilator reactivity of skeletal muscle arterioles of OZR in response to reduced $\mathrm{PO}_{2}$ compared to responses in LZR is due to a reduction in the vascular production of $\mathrm{PGI}_{2}$ and an increased vascular production of $\mathrm{TxA}_{2}$ as a result of the acute reductions in oxygen tension. Further, these effects on $\mathrm{PGI}_{2}$ and $\mathrm{TxA}_{2}$ production will be 
the result of an elevation in vascular oxidant stress, altering arachidonic acid metabolism within arterioles of OZR.

\section{MATERIALS AND METHODS}

Animals: 15-17 week old male lean and obese Zucker rats (LZR and OZR, respectively) were used for all experiments. Rats were fed standard chow and tap water ad libitum, and all protocols received prior IACUC approval. After an overnight fast, rats were anesthetized with injections of sodium pentobarbital (50 $\left.\mathrm{mg}^{\circ} \mathrm{kg}^{-1} \mathrm{i} . \mathrm{p}.\right)$, and received tracheal intubation to facilitate maintenance of a patent airway. In all rats, a carotid artery and an external jugular vein were cannulated for determination of arterial pressure and for intravenous infusion of supplemental anesthetic, if necessary. While under anesthetic, an aliquot of blood was drawn from the jugular vein to be used for the biochemical determination of plasma glucose (Freestyle; Abbott Diabetes Care, Alameda, CA), insulin (Linco; St. Charles, MO), and nitrotyrosine concentrations (Linco; St. Charles, MO) as well as a plasma lipid profile (Stanbio; Boerne, TX) from each animal.

Preparation of Isolated Skeletal Muscle Resistance Arterioles: In anesthetized rats, the intramuscular continuation of the gracilis artery was identified and surgically removed. Arterioles were placed in a heated chamber $\left(37^{\circ} \mathrm{C}\right)$ that allowed the vessel lumen and exterior to be perfused and superfused, respectively, with physiological salt solution (PSS; equilibrated with $21 \% \mathrm{O}_{2}, 5 \% \mathrm{CO}_{2} ; 74 \% \mathrm{~N}_{2}$ ) from separate reservoirs. Vessels were cannulated at both ends and were secured to inflow and outflow glass micropipettes connected to a reservoir perfusion system allowing intralumenal pressure and gas concentrations to be controlled. Arterioles were extended to their in situ length and were equilibrated at $80 \%$ of the animal's mean arterial pressure (Table 1). Vessel 
diameter was measured using television microscopy and an on-screen video micrometer, and all mechanical responses of arterioles were assessed under pressurized conditions with no flow through the arteriolar lumen.

Subsequent to the initial equilibration period, the reactivity of isolated arterioles was assessed following challenge with reduced $\mathrm{PO}_{2}\left(\Delta \mathrm{PO}_{2}\right.$ from $\sim 135 \mathrm{mmHg}\left[21 \% \mathrm{O}_{2}\right]$ - $\left.\sim 45 \mathrm{mmHg}\left[0 \% \mathrm{O}_{2}\right]\right)$ under an array of physiological and pharmacological conditions (described below). In an additional series of experiments, isolated arterioles were also challenged with increasing concentrations of the selective thromboxane $A_{2}$ mimetic $U$ $46619\left(10^{-12} \mathrm{M}-10^{-8} \mathrm{M}\right.$; Biomol) and prostacyclin $\left(\mathrm{PGI}_{2} \cdot \mathrm{Na} ; 10^{-12} \mathrm{M}-10^{-8} \mathrm{M}\right.$; Biomol) to determine the intrinsic sensitivity of microvessels to these stimuli.

Removal of the arteriolar endothelium was accomplished by passing an air bolus through the perfusate line into the isolated microvessel, the efficacy of which was determined from a loss of all dilator reactivity in response to application of $10^{-6} \mathrm{M}$ acetylcholine. To assess the contribution of nitric oxide production or the generation of metabolites via cyclooxygenase as mediators of arteriolar reactivity, isolated vessels were treated with the nitric oxide synthase inhibitor $L-N^{G}$-nitroarginine methyl ester (LNAME; $10^{-4} \mathrm{M}$; Sigma) or the cyclooxygenase inhibitor indomethacin (INDO; $10^{-5} \mathrm{M}$; Sigma), respectively. To antagonize vascular $\mathrm{PGH}_{2} / \mathrm{T} \times \mathrm{A}_{2}$ receptors, vessels were treated with SQ-29548 $\left(10^{-5} \mathrm{M}\right.$; Biomol), while inhibition of thromboxane synthase was accomplished using carboxyheptyl imidazole $\left(\mathrm{CHI} ; 10^{-5} \mathrm{M}\right.$; Biomol). To reduce vascular oxidant stress, arterioles were treated with polyethylene glycol-superoxide dismutase (PEG-SOD; $200 \mathrm{U} / \mathrm{ml}$; Sigma). 
Determination of Vascular Metabolites of Arachidonic Acid: Vascular production of 6keto-prostaglandin $\mathrm{F}_{1 \alpha}$ (6-keto-PGF$F_{1 \alpha}$; the stable breakdown product of $\mathrm{PGI}_{2}$; Refs. 18,23 ), and 11-dehydro-thromboxane $\mathrm{B}_{2}$ (11-dehydro- $\mathrm{Tx}_{2}$; the stable plasma breakdown product of $\mathrm{TxA}_{2} ;$ Ref. 5) in response to challenge with reduced oxygen tension was assessed using pooled conduit arteries (femoral, saphenous, iliac) from LZR and OZR. Pooled vessels from each animal were incubated in microcentrifuge tubes in $1 \mathrm{ml}$ of physiological salt solution for 30 minutes under control conditions $\left(21 \% \mathrm{O}_{2}\right)$. After this time, the superfusate was removed, stored in a new microcentrifuge tube and frozen in liquid $\mathrm{N}_{2}$, while a new aliquot of PSS was added to the vessels and the equilibration gas was switched to $0 \% \mathrm{O}_{2}$ for the subsequent 30 minutes. After the second 30 minute period, this new PSS was transferred to a fresh tube, frozen in liquid $\mathrm{N}_{2}$ and stored at $-80^{\circ} \mathrm{C}$. Metabolite release by the vessels was determined using commercially available EIA kits for 6-keto-PGF ${ }_{1 \alpha}$ and 11dehydro- $\mathrm{TxB}_{2}$ (Cayman).

Data and Statistical Analyses: Active tone of individual arterioles at the equilibration pressure was calculated as $\left(\Delta D / D_{\max }\right) \cdot 100$, where $\Delta D$ is the diameter increase from rest in response to $\mathrm{Ca}^{2+}$-free PSS, and $\mathrm{D}_{\max }$ is the maximum diameter measured at the equilibration pressure in $\mathrm{Ca}^{2+}$-free PSS.

Mechanical responses of isolated arterioles following challenge with increasing concentrations of thromboxane or prostacyclin were fit with the three-parameter logistic equation:

$$
y=\min +\left[\frac{\max -\min }{1+10^{\log E C_{50}-x}}\right]
$$


where $y$ represents the change in arteriolar diameter, "min" and "max" represent the minimum and maximum bounds, respectively, of the change in arteriolar diameter with increasing agonist concentration, $x$ is the logarithm of the agonist concentration and $\log E C_{50}$ represents the logarithm of the agonist concentration $(x)$ at which the response $(y)$ is halfway between the minimum and maximum bounds.

Data are presented as mean \pm SEM. Statistically significant differences in the magnitude of hypoxic dilation, vascular production of 6-keto-PGF ${ }_{1 \alpha}$ and 11-dehydro-TxB and the calculated parameters describing the thromboxane or prostacyclin concentrationresponse relationships were determined using analysis of variance (ANOVA). In all cases, Student-Newman-Keuls post hoc test was used when appropriate and $p<0.05$ was taken to reflect statistical significance.

\section{RESULTS}

Table 1 presents baseline characteristics of LZR and OZR in the present study. At 15-17 weeks of age, OZR demonstrated profound obesity, severe insulin resistance, and dyslipidemia characterized by moderate hypercholesterolemia and severe hypertriglyceridemia. In addition, OZR also presented a moderate elevation in mean arterial pressure as compared to LZR. Plasma levels of nitrotyrosine, a protein marker of chronic elevations in oxidant stress, were significantly elevated in OZR compared to LZR. With regard to basal vascular tone, isolated arterioles from both rat strains demonstrated a comparable resting active diameter, although passive diameter was reduced in OZR versus LZR. However, this remodeling of the vessel wall did not translate into a difference in calculated active tone between the strains. 
Data summarizing hypoxic dilation of resistance arterioles from LZR and OZR are presented in Figure 1. As shown in Panel A, arterioles from OZR exhibited a blunted dilator response to reduced $\mathrm{PO}_{2}$ compared to vessels from LZR. However, arteriolar reactivity to reduced oxygen tension was abolished in both groups following removal of the vascular endothelium. Panel B presents data describing the contribution of nitric oxide synthase (NOS) and cyclooxygenase (COX) products to arteriolar dilation in response to reduced $\mathrm{PO}_{2}$ in LZR and OZR. While NOS inhibition has a consistently minor, albeit statistically insignificant, blunting of hypoxic dilation in arterioles of LZR, treatment of vessels with L-NAME had no discernible impact on this response in OZR. In contrast, incubation of vessels with indomethacin dramatically reduced hypoxic dilation in arterioles from both strains. Combined treatment with L-NAME and indomethacin abolished all vascular responses to reduced $\mathrm{PO}_{2}$ in both LZR and OZR. Panel $\mathrm{C}$ presents the impact of pre-treatment of arterioles with the anti-oxidant PEG-SOD on both the magnitude of hypoxic dilation in these vessels and on the contributions from NOS and COX. Treatment of vessels with PEG-SOD had no significant impact on either the magnitude of hypoxic dilation or on the contribution of NOS and COX products to this response in LZR. In contrast, following incubation with PEG-SOD, arterioles from OZR exhibited an improved dilation in response to reduced $\mathrm{PO}_{2}$, and this enhanced reactivity was entirely dependent on COX metabolism.

Figure 2 presents data describing the production of 6 -keto-PGF $F_{1 \alpha}$ (the stable breakdown product of $\mathrm{PGI}_{2}$ ) and 11-dehydro- $\mathrm{TxB}_{2}$ (the stable breakdown product of $\mathrm{TxA}_{2}$ ) from pooled vessels of $L Z R$ and OZR in response to reduced $\mathrm{PO}_{2}$. As shown in Panel $A$, vessels from both LZR and OZR demonstrated a significant increase in 6-keto-PGF ${ }_{1 \alpha}$ 
production in response to reduced $\mathrm{PO}_{2}$, although this increase was greater in LZR as compared to OZR. In both cases, treatment of vessels with indomethacin abolished 6keto-PGF $F_{1 \alpha}$ production in response to reduced oxygen tension. With regard to the vascular production of $\mathrm{TXA}_{2}$, vessels from both LZR and OZR exhibited a significant increase in 11dehydro- $\mathrm{Tx}_{2}$ production following exposure to reduced $\mathrm{PO}_{2}$, although this level of production was dramatically elevated in vessels from OZR (Panel B). In both cases, production of 11-dehydro- $\mathrm{TxB}_{2}$ was abolished following treatment of vessels with either indomethacin or $\mathrm{CHI}$, suggesting that all production of $\mathrm{TxA}_{2}$ was mediated via the actions of thromboxane synthase distal to COX.

Data describing the production of 6-keto-PGF ${ }_{1 \alpha}$ and 11-dehydro- $\mathrm{TxB}_{2}$ in response to reduced $\mathrm{PO}_{2}$ in vessels from OZR subsequent to pre-treatment with PEG-SOD are summarized in Figure 3. Treatment of vessels with PEG-SOD did not alter 6-keto$\mathrm{PGF}_{1 \alpha}$ production in vessels from OZR compared to levels in untreated vessels (Panel A). However, as compared to levels determined in untreated vessels, incubation of arteries from OZR with PEG-SOD blunted the enhanced production of 11-dehydro-TxB 2 (Panel B) Treatment of vessels with indomethacin abolished reduced $\mathrm{PO}_{2}$-induced production of 6keto-PGF ${ }_{1 \alpha}$ and 11-dehydro- $\mathrm{TxB}_{2}$.

Figure 4 presents data summarizing the dilator responses of arterioles from LZR and OZR in response to reduced $\mathrm{PO}_{2}$ following antagonism of either the $\mathrm{PGH}_{2} / \mathrm{TxA}_{2}$ receptor with SQ-29548 or thromboxane synthase with $\mathrm{CHI}$. While neither of these pharmacological interventions had a consistent and significant impact on response in vessels from LZR, both SQ-29548 and $\mathrm{CH}$ significantly increased hypoxic dilation in arterioles from OZR. 
Arteriolar reactivity in response to challenge with increasing concentrations of thromboxane or prostacyclin is summarized in Figure 5. Arteriolar constriction in response to increasing concentrations of thromboxane was not different between LZR and OZR, and neither $E C_{50}$ nor maximum bound were impacted in response to pre-treatment of the vessels with PEG-SOD (Panel A). Further, arteriolar responses to thromboxane were abolished in vessels from both animal strains following incubation of the vessels with SQ29548 (Panel B). With increasing concentrations of prostacyclin, arterioles from OZR exhibited a blunted dilator response compared to that determined in arterioles from LZR, and treatment of vessels with PEG-SOD significantly improved dilator reactivity to prostacyclin, although not to the level determined in LZR (Panel C). EC 50 was not significantly different between groups with regard to vascular reactivity in response to increasing concentrations of $\mathrm{PGI}_{2}$ (data not shown)

\section{DISCUSSION}

Inherent within the development of the multi-pathology state that defines the metabolic syndrome is an array of alterations to vascular and microvascular structure and function that have the potential to profoundly impact the perfusion of tissues and organs, as well as the contributing mechanisms which comprise this integrated process. Previous studies have clearly demonstrated that alterations to the patterns of vasodilator $(8,12)$ and vasoconstrictor $(30)$ reactivity, as well as vessel wall remodeling $(28,29)$ and a reduction in microvessel density (7) all represent avenues through which vascular function and tissue perfusion can be compromised. The purpose of the present study was to build on our previous observation of an impaired dilation of skeletal muscle resistance arterioles of OZR 
in response to acute reductions in oxygen tension, and the possible contributing role of elevated vascular oxidant stress in this process (9).

Comparable to our observations in the first study, hypoxic dilation of skeletal muscle resistance arterioles from OZR was significantly reduced compared to that determined in LZR (Figure 1, Panel A). While this response was abolished following removal of the endothelium in both rat strains, comparable to the complete endothelium-dependence of hypoxic dilation in other rodent models $(14,19,20)$, our results also support previous observations of a strong dependence on COX products in mediating hypoxic dilation of these vessels (18-20). Interestingly, in our initial study we determined a small, but significant role for NOS activity in mediating hypoxic dilation in arterioles from LZR. In the present study this effect was not statistically significant, suggesting that it may represent a minor contributor to arteriolar dilation in response to reduced oxygen tension in LZR. However, while the magnitude of this response is relatively minor in LZR, both the results from the present study and our previous one (9) indicate that this is completely lost with the development of the metabolic syndrome in OZR. Additionally, pre-treatment of arterioles from OZR with the oxidative free radical scavenger PEG-SOD to lower the elevated oxidant stress in these animals resulted in a significant improvement to hypoxic dilation in the obese rats (Figure 1, Panel C). This improvement to hypoxic dilation was confined to activity involving COX only, as treatment with indomethacin abolished hypoxic dilation in OZR, and treatment with L-NAME was without effect.

Recent studies from Hester's group suggest that functional dilation (i.e., arteriolar dilation in response to elevated metabolic demand) is impaired in OZR as a result of alterations to arachidonic acid metabolism which can result in activation of thromboxane 
receptors causing a competing constrictor influence which acts to partially constrain dilator responses $(32,33)$. Given that both functional dilation for in situ spintotrapezius muscle of LZR (11) and hypoxic dilation of skeletal muscle arterioles of LZR (9) has previously been shown to be strongly dependent on the vascular production of $\mathrm{PGI}_{2}$, we sought to determine if an alteration in arachidonic acid metabolism, resulting in an increased vascular production of thromboxane or a decreased production of prostacyclin may contribute to this impaired reactivity in arterioles of OZR. As shown in Figure 2, arteries from OZR exhibited a significant increase in the production of $\mathrm{PGI}_{2}$ (estimated from measurements of 6-keto-PGF ${ }_{1 \alpha}$ ) in response to reductions in oxygen tension. While this was not as robust a response as that determined for arteries of LZR, it is unclear if this degree of attenuation in $\mathrm{PGI}_{2}$ production in OZR is sufficient to manifest itself as a blunted mechanical response. In contrast, vascular production of $\mathrm{TxA}_{2}$ (estimated from measurements of 11-dehydro-TxB $\mathrm{B}_{2}$, mildly elevated in arteries of LZR during reduced oxygen tension, was dramatically increased in vessels from OZR following exposure to reduced $\mathrm{PO}_{2}$. Finally, while treatment of arteries from both strains with indomethacin abolished production of $\mathrm{PGI}_{2}$ and $\mathrm{TxA}_{2}$ in response to reduced oxygen tension, the treatment of vessels of OZR with $\mathrm{CHI}$, an inhibitor of thromboxane synthase, severely reduced thromboxane production in vessels from OZR (Figure 2, Panel B). These results suggest that reduced $\mathrm{PO}_{2}$ causes an increased production of $\mathrm{TXA}_{2}$ from vessels of OZR, mediated via thromboxane synthase, and that this may compete with the dilator influences of vascular production of $\mathrm{PGI}_{2}$. However, it should be emphasized that these results must be interpreted cautiously, as the production of metabolites of arachidonic acid was assessed using conduit arteries and not 
resistance arterioles, while the reverse is true with regard to the study of the mechanical responses.

In order to better understand the impact of elevated vascular oxidant stress on the impaired dilator responses to reduced $\mathrm{PO}_{2}$ in vessels of OZR, we treated vessels with PEG-SOD prior to exposure to reduced $\mathrm{PO}_{2}$ and determined the levels of 6-keto-PGF ${ }_{1 \alpha}$ and 11-dehydro-TxB $\mathrm{B}_{2}$ in the incubation superfusate (Figure 3 ). These data suggest that while oxidant stress does not play a significant role in any reduction in the vascular production of prostacyclin (as the addition of PEG-SOD was without effect), elevated vascular oxidant stress may contribute to the increased production of thromboxane by these vessels, as incubation with the anti-oxidant significantly reduced the levels of 11dehydro- $\mathrm{TxB}_{2}$ in the superfusate. However, this effect was not complete, as the levels of 11-dehydro-TxB 2 production remained elevated in the superfusate despite the presence of PEG-SOD, indicating that additional factors may contribute to thromboxane production in vessels of OZR which are independent of the effects of acute reduction in vascular oxidant stress. This observation of a role for oxidant stress in shifting arachidonic acid metabolism toward an increased production of thromboxane has been identified previously $(2,3,34)$, and the results of the present study suggest that a comparable effect may be occurring in the vasculature of OZR, with the net result of a blunted vascular response to stimuli that are dependent on $\mathrm{PGI}_{2}$ production for their full manifestation.

To better evaluate this statement, isolated skeletal muscle resistance arterioles from LZR and OZR were exposed to acute reductions in oxygen tension under control conditions and in response to either $\mathrm{PGH}_{2} / \mathrm{TXA}_{2}$ receptor blockade with SQ-29548 or thromboxane synthase inhibition with $\mathrm{CHI}$ (Figure 4). Although these pharmacological 
challenges had no consistent impact on hypoxic dilation in arterioles from LZR, both interventions resulted in improved dilator reactivity in response to reduced $\mathrm{PO}_{2}$ in arterioles of OZR. Taken with the previous results, these data clearly suggest that an increased production of $\mathrm{TxA}_{2}$ contributes to the impaired hypoxic dilation in arterioles of OZR. However, the data presented in Figure 4 do not allow for discrimination between increased vascular production of $\mathrm{TxA}_{2}$, an increased vascular sensitivity to produced $\mathrm{TxA}_{2}$, or an altered vascular sensitivity to produced $\mathrm{PGI}_{2}$.

In order to assess this final issue, isolated arterioles from both rat strains were challenged with increasing concentrations of thromboxane or prostacyclin (Figure 5). Vasoconstrictor reactivity to thromboxane was very similar between arterioles of LZR and OZR, and in both cases constrictor responses to thromboxane were largely independent of oxidant stress (i.e., no identifiable impact of treatment with PEG-SOD) and were abolished by blockade of the $\mathrm{PGH}_{2} / \mathrm{TxA}_{2}$ receptor. These observations suggest that the vascular sensitivity to thromboxane is not significantly impacted by the presence of the metabolic syndrome. In contrast, vascular reactivity to prostacyclin was significantly reduced in arterioles of OZR and this impairment was blunted following a reduction in oxidant stress with PEG-SOD. Whether this impaired response to $\mathrm{PGI}_{2}$ represents oxidant radical degradation of prostacyclin (leading to the production of isoprostanes), altered function at the level of the prostacyclin receptor, the impact of elevated oxidant stress on the intracellular signaling cascade distal to the receptor, or a combination of these effects remains to be determined.

In summary, with the evolution of the metabolic syndrome in obese Zucker rats, the dilator responses of skeletal muscle resistance arterioles following acute reductions in 
oxygen tension are significantly attenuated. Both biochemical and pharmacological evidence suggests that this impaired dilator reactivity may be the result of an increase in vascular production of thromboxane with reduced $\mathrm{PO}_{2}$ which could represent a constrictor influence which competes against the dilator effects of prostacyclin (the production of which appears to be largely intact). Normalizing vascular oxidant stress blunts the increased reduced $\mathrm{PO}_{2}$-induced production of thromboxane in vessels from OZR and also increases the responsiveness of arterioles of OZR to exogenously supplied prostacyclin, thus leading to an improvement in the mechanical response of the vessel to reduced oxygen tension. The present study provides no compelling evidence that skeletal muscle arteriolar sensitivity to thromboxane is altered with the progression of the metabolic syndrome.

\section{ACKNOWLEDGEMENTS}

The authors gratefully acknowledge the support provided through the "Translational Research Initiative: Cardiorespiratory Health in Appalachia - from Mechanisms to Policy" (TRI-CHAMP) at the West Virginia University Health Sciences Center in the

performance of this study. This study was supported by the American Heart Association (SDG 0330194N) and the National Institutes of Health (R01 DK64668). 
Reference List

1. Asghar, M., E. Monjok, G. Kouamou, S.E. Ohia, D. Bagchi and M.F. Lokhandwala. Super CitriMax (HCA-SX) attenuates increases in oxidative stress, inflammation, insulin resistance, and body weight in developing obese Zucker rats. Mol Cell Biochem. 304:93-99, 2007.

2. Bachschmid, M., S. Schildknecht and V. Ullrich. Redox regulation of vascular prostanoid synthesis by the nitric oxide-superoxide system. Biochem Biophys Res Commun. 338:536-542, 2005.

3. Bachschmid, M., S. Thurau, M.H. Zou and V Ullrich. Endothelial cell activation by endotoxin involves superoxide/NO-mediated nitration of prostacyclin synthase and thromboxane receptor stimulation. FASEB J. 17:914-916, 2003.

4. Bouvet, C., E.B. de Chantemèle, A.L. Guihot, E. Vessières, A. Bocquet, O. Dumont, A. Jardel, L. Loufrani, P. Moreau and D. Henrion. Flow-induced remodeling in resistance arteries from obese Zucker rats is associated with endothelial dysfunction. Hypertension. 50:248-254, 2007.

5. Catella, F., D. Healy, J.A. Lawson and G.A. FitzGerald. 11-Dehydrothromboxane B2: a quantitative index of thromboxane A2 formation in the human circulation. Proc Natl Acad Sci U S A. 83:5861-5865, 1986. 
6. Dominguez, J., P. Wu, C.S. Packer, C. Temm and K.J. Kelly. Lipotoxic and inflammatory phenotypes in rats with uncontrolled metabolic syndrome and nephropathy. Am J Physiol Renal Physiol. 293:F670-F679, 2007.

7. Frisbee, J.C., J.B. Samora, J. Peterson and R. Bryner. Exercise training blunts microvascular rarefaction in the metabolic syndrome. Am J Physiol Heart Circ Physiol. 291:H2483-H2492, 2006.

8. Frisbee, J.C. and M.D. Delp. Vascular function in the metabolic syndrome and the effects on skeletal muscle perfusion: lessons from the obese Zucker rat. Essays Biochem. 42:1451-61, 2006.

9. Frisbee, J.C. Impaired dilation of skeletal muscle microvessels to reduced oxygen tension in diabetic obese Zucker rats. Am J Physiol Heart Circ Physiol. 281:H1568H1574, 2001.

10. Guerre-Millo, M. Regulation of ob gene and overexpression in obesity. Biomed Pharmacother. 51:318-323, 1997.

11. Hammer, L.W., A.L. Ligon and R.L. Hester. Differential inhibition of functional dilation of small arterioles by indomethacin and glibenclamide. Hypertension. 37:599-603, 2001. 
12. Hodnett, B.L. and R.L. Hester. Regulation of muscle blood flow in obesity. Microcirculation. 14:273-288, 2007.

13. Jonk, A.M., A.J. Houben, R.T. de Jongh, E.H. Serné, N.C. Schaper and C.D. Stehouwer. Microvascular dysfunction in obesity: a potential mechanism in the pathogenesis of obesity-associated insulin resistance and hypertension. Physiology (Bethesda). 22:252-260, 2007.

14.Kerkhof, C.J., E.N. Bakker and P. Sipkema. Role of cytochrome P-450 4A in oxygen sensing and $\mathrm{NO}$ production in rat cremaster resistance arteries. Am J Physiol. 277:H1546-H1552, 1999.

15.Kim, J.A., M. Montagnani, K.K. Koh and M.J. Quon. Reciprocal relationships between insulin resistance and endothelial dysfunction: molecular and pathophysiological mechanisms. Circulation. 113:1888-1904, 2006.

16. Knudson, J.D., U.D. Dincer, I.N. Bratz, M. Sturek, G.M. Dick and J.D. Tune. Mechanisms of coronary dysfunction in obesity and insulin resistance. Microcirculation. 14:317-338, 2007.

17. Lesniewski, L.A., A.J. Donato, B.J. Behnke, C.R. Woodman, M.H. Laughlin, C.A. Ray and M.D. Delp. Decreased NO signaling leads to enhanced 
vasoconstrictor responsiveness in skeletal muscle arterioles of the ZDF rat prior to overt diabetes and hypertension. Am J Physiol Heart Circ Physiol. 294:H1840H1850, 2008.

18. Liu, Y., K.T. Fredricks, R.J. Roman and J.H. Lombard. Response of resistance arteries to reduced PO2 and vasodilators during hypertension and elevated salt intake. Am J Physiol. 273:H869-H877, 1997.

19. Lombard, J.H., Y. Liu, K.T. Fredricks, D.M. Bizub, R.J. Roman and N.J. Rusch. Electrical and mechanical responses of rat middle cerebral arteries to reduced PO2 and prostacyclin. Am J Physiol. 276:H509-H516, 1999.

20. Messina, E.J., D. Sun, A. Koller, M.S. Wolin and G. Kaley. Role of endotheliumderived prostaglandins in hypoxia-elicited arteriolar dilation in rat skeletal muscle. Circ Res. 71:790-796, 1992.

21. Naik, J.S., L. Xiang and R.L. Hester. Enhanced role for RhoA-associated kinase in adrenergic-mediated vasoconstriction in gracilis arteries from obese Zucker rats. Am J Physiol Regul Integr Comp Physiol. 290:R154-R161, 2006.

22. Naka, Y., L.G. Bucciarelli, T. Wendt, L.K. Lee, L.L. Rong, R. Ramasamy, S.F. Yan and A.M. Schmidt. RAGE axis: Animal models and novel insights into the 
vascular complications of diabetes. Arterioscler Thromb Vasc Biol. 24:1342-1349, 2004.

23. Nies, A.S. Prostaglandins and the control of the circulation. Clin Pharmacol Ther. 39:481-488, 1986.

24. Rosin, B.L. The progression of cardiovascular risk to cardiovascular disease. Rev Cardiovasc Med. 8:S3-S8, 2007.

25. Saely C.H., P. Rein and H. Drexel. The metabolic syndrome and risk of cardiovascular disease and diabetes: experiences with the new diagnostic criteria from the International Diabetes Federation. Horm Metab Res. 39:642-650, 2007.

26.Shammas, N.W. Epidemiology, classification, and modifiable risk factors of peripheral arterial disease. Vasc Health Risk Manag. 3:229-234, 2007.

27.Shammas, N.W. and E.J. Dippel. Evidence-based management of peripheral vascular disease. Curr Atheroscler Rep. 7:358-363, 2005.

28.Sista, A.K., M.K. O'Connell, T. Hinohara, S.S. Oommen, B.E. Fenster, A.J. Glassford, E.A. Schwartz, C.A. Taylor, G.M. Reaven and PS Tsao. Increased aortic stiffness in the insulin-resistant Zucker fa/fa rat. Am J Physiol Heart Circ Physiol. 289:H845-H851, 2005. 
29. Stepp, D.W., D.M. Pollock and J.C. Frisbee. Low-flow vascular remodeling in the metabolic syndrome X. Am J Physiol Heart Circ Physiol. 286:H964-H970, 2004.

30.Stepp, D.W. and J.C. Frisbee. Augmented adrenergic vasoconstriction in hypertensive diabetic obese Zucker rats. Am J Physiol Heart Circ Physiol. 282:H816-H820, 2002.

31.Toblli, J.E., G. Cao, G. DeRosa, F. Di Gennaro and P. Forcada. Angiotensinconverting enzyme inhibition and angiogenesis in myocardium of obese Zucker rats. Am J Hypertens. 17:172-180, 2004.

32.Xiang, L., J. Dearman, S.R. Abram, C. Carter and R.L. Hester. Insulin resistance and impaired functional vasodilation in obese Zucker rats. Am J Physiol Heart Circ Physiol. 294:H1658-H1666, 2008.

33. Xiang, L., J.S. Naik, B.L. Hodnett and R.L. Hester. Altered arachidonic acid metabolism impairs functional vasodilation in metabolic syndrome. Am J Physiol Regul Integr Comp Physiol. 290:R134-R138, 2006.

34.Zou, M.H. Peroxynitrite and protein tyrosine nitration of prostacyclin synthase. Prostaglandins Other Lipid Mediat. 82:119-127, 2007. 
Table 1. Baseline characteristics of LZR and OZR and for isolated arterioles in the present study. * $\mathrm{p}<0.05$ vs. LZR.

\begin{tabular}{|c|c|c|}
\hline & LZR & OZR \\
\hline Mass (g) & $361 \pm 7$ & $664 \pm 9^{*}$ \\
\hline $\mathrm{MAP}(\mathrm{mmHg})$ & $108 \pm 4$ & $124 \pm 4^{*}$ \\
\hline$[\text { Glucose }]_{\text {blood }}(\mathrm{mg} / \mathrm{dl})$ & $102 \pm 5$ & $184 \pm 11^{*}$ \\
\hline$[\text { Insulin }]_{\text {plasma }}(\mathrm{ng} / \mathrm{ml})$ & $1.3 \pm 0.3$ & $7.5 \pm 0.5^{*}$ \\
\hline$[\text { Total Cholesterol }]_{\text {plasma }}(\mathrm{mg} / \mathrm{dl})$ & $89 \pm 9$ & $137 \pm 11^{*}$ \\
\hline$[\text { Triglycerides }]_{\text {plasma }}(\mathrm{mg} / \mathrm{dl})$ & $154 \pm 10$ & $367 \pm 22^{*}$ \\
\hline Nitrotyrosine $_{\text {plasma }}(\mathrm{ng} / \mathrm{ml})$ & $15 \pm 4$ & $58 \pm 7^{*}$ \\
\hline Inner Diameter - Active $(\mu \mathrm{m})$ & $104 \pm 4$ & $102 \pm 5$ \\
\hline Inner Diameter - Passive $(\mu \mathrm{m})$ & $172 \pm 5$ & $156 \pm 4^{*}$ \\
\hline Active Tone (\%) & $39 \pm 2$ & $35 \pm 3$ \\
\hline
\end{tabular}




\section{FIGURE LEGENDS}

Figure 1. Dilator reactivity of isolated skeletal muscle resistance arterioles of LZR and OZR in response to acute reductions in oxygen tension. Data, presented as mean $\pm S E M$, are shown for arterioles under control conditions and following removal of the vascular endothelium using air bolus perfusion (Panel A), inhibition of nitric oxide synthase with LNAME and/or inhibition of cyclooxygenase with indomethacin (Panel B), treatment with LNAME and/or indomethacin following incubation of the arteriole with the anti-oxidant PEGSOD (Panel C). Please see manuscript text for complete details. ${ }^{*} p<0.05$ vs. Control responses in that strain; $\uparrow p<0.05$ vs. responses in LZR Control; $\ddagger p<0.05$ vs. responses in OZR + PEG-SOD

Figure 2. Vascular production of 6-keto-PGF ${ }_{1 \alpha}\left(P a n e l A\right.$; as an estimate of $\mathrm{PGI}_{2}$ ) and 11dehydro-TxB $B_{2}\left(\right.$ Panel $B$; as an estimate of $\mathrm{TxA}_{2}$ ) by pooled arteries of LZR and OZR in response to an acute reduction in oxygen tension. Data, presented as mean $\pm S E M$, are shown for arteries under control conditions, and following pharmacological inhibition of cyclooxygenases with indomethacin, and for 11-dehydro- $\mathrm{TxB}_{2}$, thromboxane synthase with CHI. ${ }^{*} p<0.05$ vs. Control $\left(21 \% \mathrm{O}_{2}\right)$ in that strain, $\dagger p<0.05$ vs. $0 \% \mathrm{O}_{2}$ in that strain; $\ddagger$ $p<0.05$ vs. responses in $\mathrm{LZR} 0 \% \mathrm{O}_{2}$.

Figure 3. Vascular production of 6-keto-PGF ${ }_{1 \alpha}\left(P a n e l A\right.$; as an estimate of $\left.\mathrm{PGI}_{2}\right)$ and 11dehydro-TxB $\left(\right.$ Panel $B$; as an estimate of $\mathrm{TxA}_{2}$ ) by pooled arteries OZR in response to an acute reduction in oxygen tension. Data, presented as mean \pm SEM, are shown for arteries under control conditions, following treatment of arteries with the anti-oxidant PEG-SOD, 
and following pharmacological inhibition of cyclooxygenases with indomethacin. ${ }^{*} p<0.05$ vs. responses under Control $\left(21 \% \mathrm{O}_{2}\right)$ conditions, $\uparrow p<0.05$ vs. responses under $0 \% \mathrm{O}_{2} ; \ddagger$ $p<0.05$ vs. responses determined under $0 \% \mathrm{O}_{2}$ conditions + PEG-SOD.

Figure 4. Dilator reactivity of isolated skeletal muscle resistance arterioles of LZR and OZR in response to acute reductions in oxygen tension. Data (mean $\pm S E M)$ are presented for each strain under control conditions, and following pharmacological inhibition of the $\mathrm{PGH}_{2} / \mathrm{TxA}_{2}$ receptor with SQ-29548 or thromboxane synthase with $\mathrm{CHI} . \quad{ }^{*} \mathrm{p}<0.05$ vs. $\mathrm{LZR}$ Control; $\uparrow p<0.05$ vs. OZR Control.

Figure 5. Vascular reactivity of isolated skeletal muscle resistance arterioles of LZR and OZR (mean $\pm S E M)$ in response to increasing concentrations of thromboxane under control conditions and following treatment of vessels with PEG-SOD (Panel A) or SQ-29548 (Panel B), and increasing concentrations of prostacyclin under control conditions and following treatment with PEG-SOD (Panel C). The term "max" represents the maximum bound (the maximum change) in vessel diameter in response to increasing concentration of either thromboxane or prostacyclin; estimated from the logistic regression equation described in the "Materials and Methods". * $p<0.05$ vs. Control responses within that strain; $\dagger p<0.05$ vs. responses in LZR under Control conditions. 


\section{FIGURES}

Figure 1.
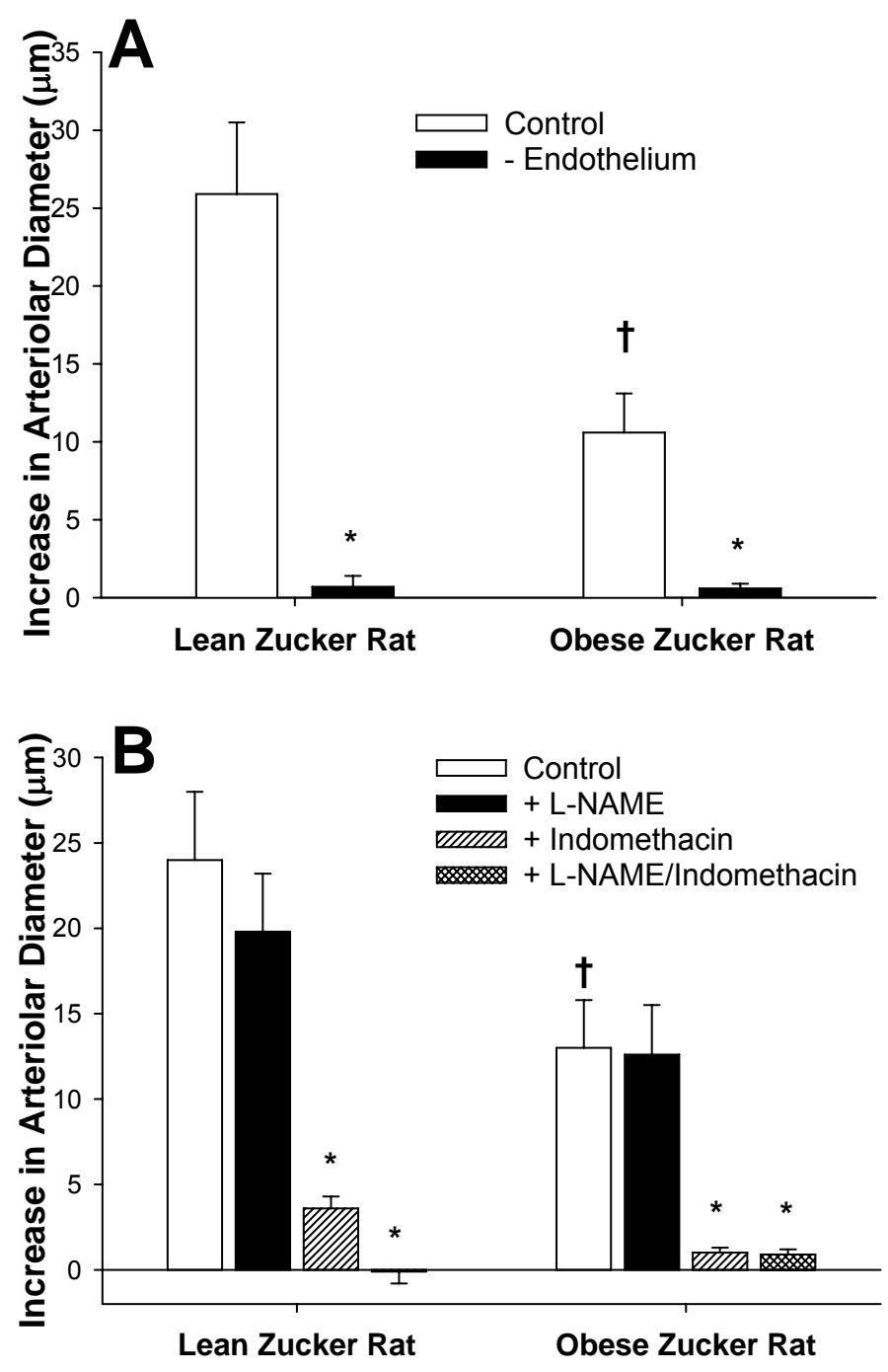


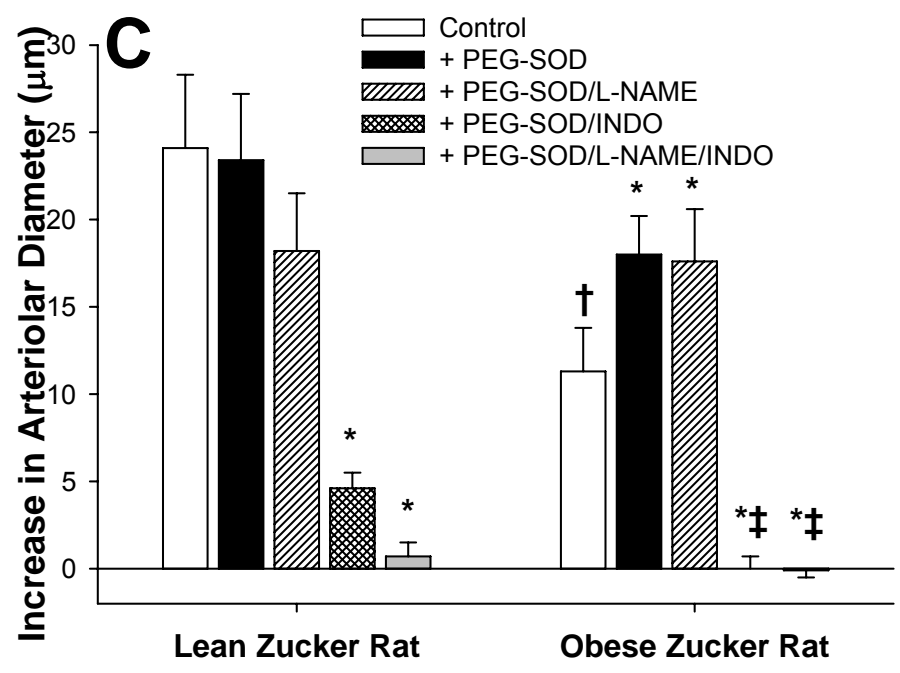


Figure 2.
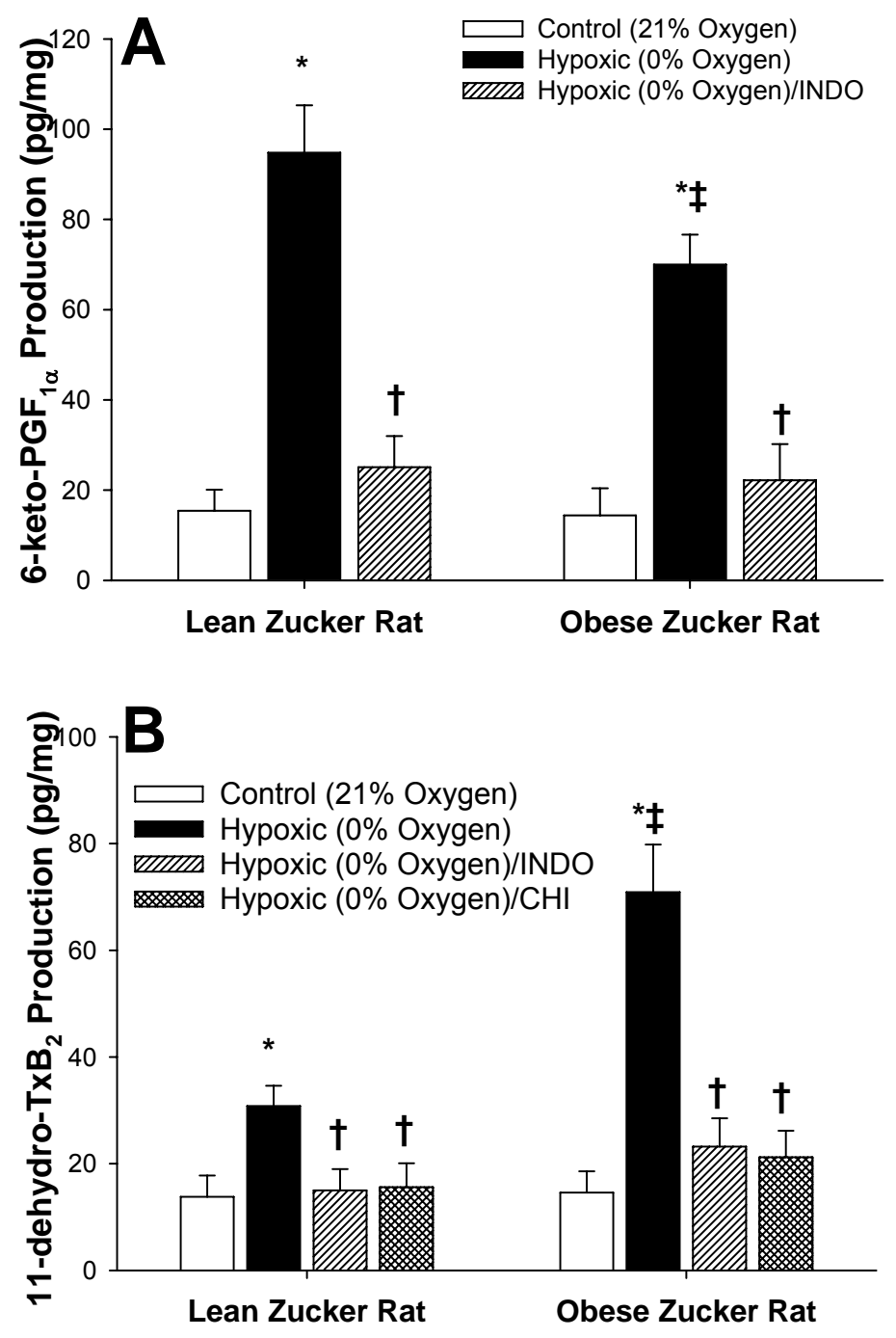
Figure 3.
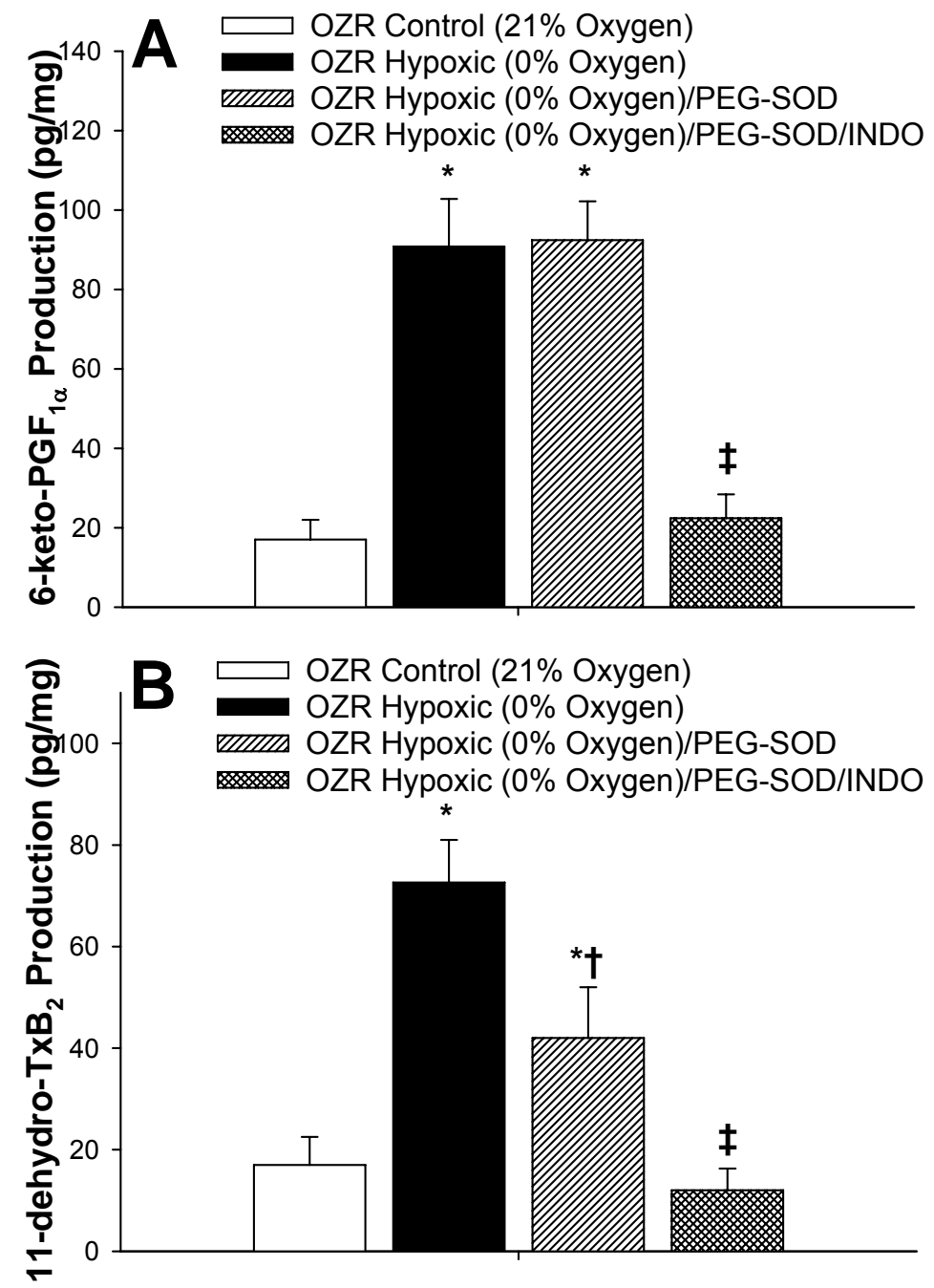
Figure 4.

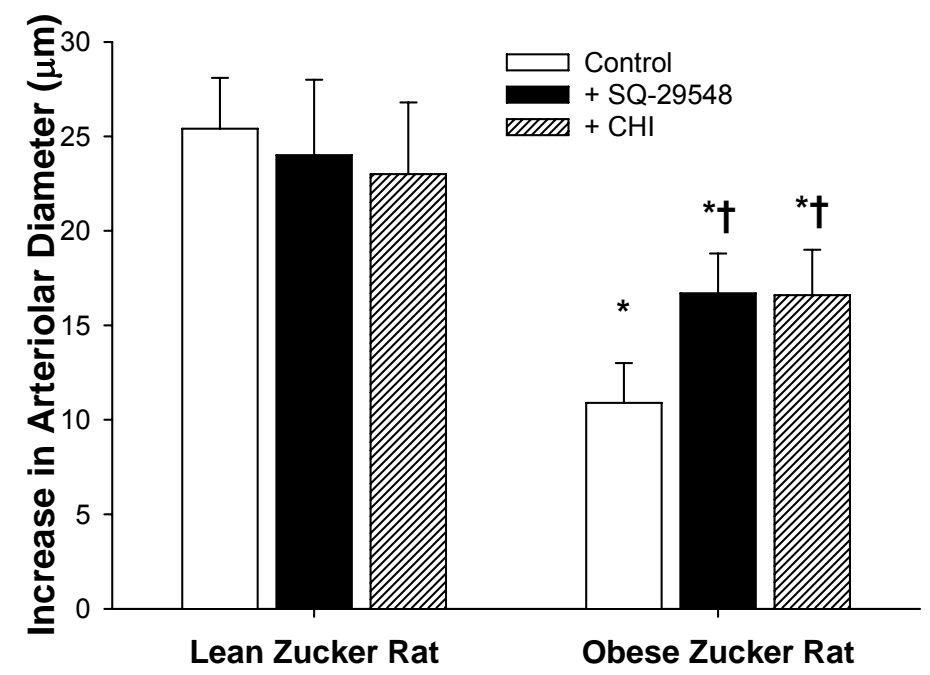




\section{Figure 5.}

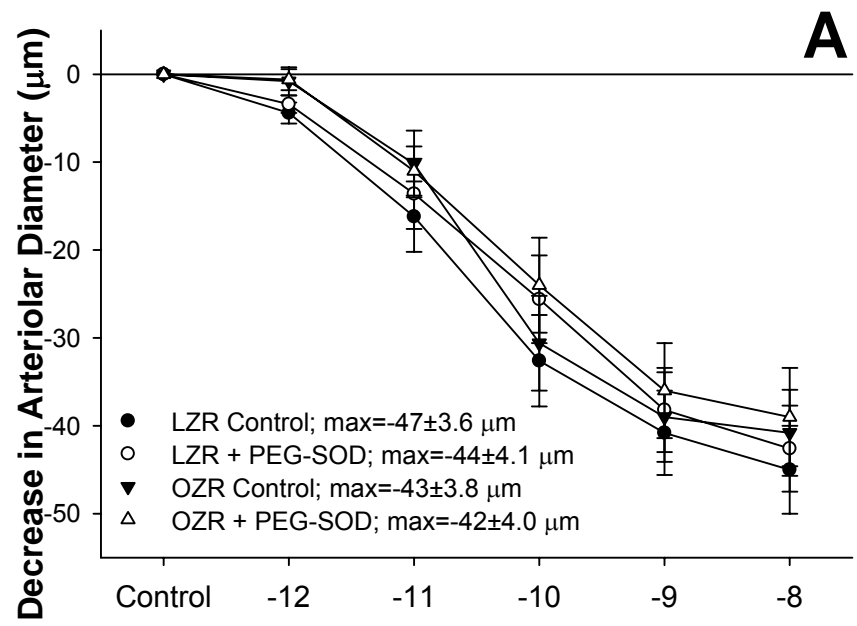

log Thromboxane Concentration (M)

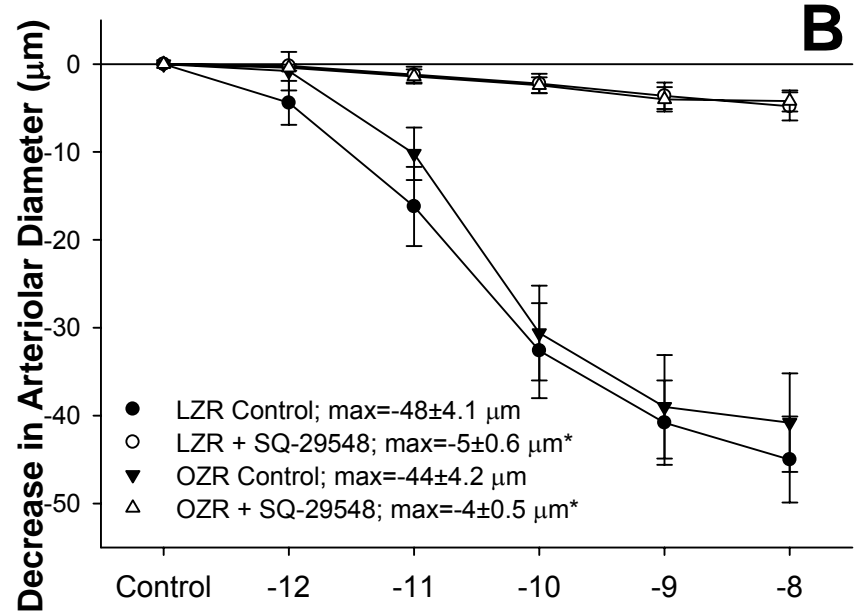

log Thromboxane Concentration (M)

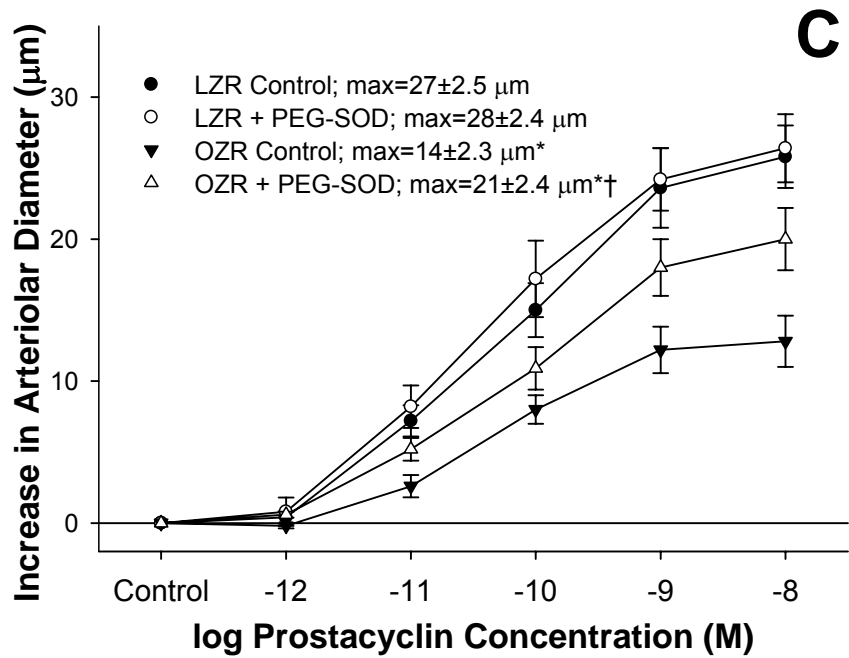




\title{
CHAPTER 3
}

\section{INCREASED ARACHIDONIC ACID-INDUCED THROMBOXANE GENERATION IMPAIRS SKELETAL MUSCLE ARTERIOLAR DILATION WITH GENETIC DYSLIPIDEMIA}

\author{
Adam. G. Goodwill ${ }^{1,4}$, Phoebe A. Stapleton ${ }^{2,4}$, Milinda E. James ${ }^{1,4}$, Alexandre C. \\ d'Audiffret $^{3,4}$ and Jefferson C. Frisbee ${ }^{1,4}$ \\ Department of Physiology and Pharmacology ${ }^{1}$, Division of Exercise Physiology ${ }^{2}$, \\ Division of Vascular and Endovascular Surgery ${ }^{3}$ and Center for Interdisciplinary \\ Research in Cardiovascular Sciences ${ }^{4}$ \\ West Virginia University School of Medicine, Morgantown, WV 26506
}

Running Head: Hypercholesterolemia and arteriolar reactivity

Key Words: skeletal muscle microcirculation, endothelium-dependent dilation, vascular reactivity, mouse models of cardiovascular disease, hypercholesterolemia

Support: This study was supported by the American Heart Association (EIA 0740129N) and the National Institutes of Health (R01 DK64668).

"This research was originally published in Microcirculation. Goodwill AG, Stapleton PA, James ME, d'Audiffret AC, Frisbee JC . Increased Arachidonic Acid-Induced Thromboxane Generation Impairs Skeletal Muscle Arteriolar Dilation with Genetic Dyslipidemia. Microcirculation. 2008 Oct;15(7):621-31. 


\section{ABSTRACT}

Objective: To determine if arachidonic acid (AA)-induced skeletal muscle arteriolar dilation is altered with hypercholesterolemia in ApoE and LDLR gene deletion mice fed normal diet. This study also determined contributors to altered AA-induced dilation between dyslipidemic mice and controls; C57/BI/6J (C57).

Methods: Gracilis muscle arterioles were isolated, with mechanical responses assessed following challenge with $A A$ under control conditions and after elements of AA metabolism pathways were inhibited. Conduit arteries from each strain were used to assess $A A$-induced production of $\mathrm{PGI}_{2}$ and $\mathrm{TxA}_{2}$.

Results: Arterioles from ApoE and LDLR exhibited a blunted dilation to AA versus C57. While responses were cyclooxygenase-dependent in all strains, inhibition of thromboxane synthase or blockade of $\mathrm{PGH}_{2} / \mathrm{TxA}_{2}$ receptors improved dilation in $\mathrm{ApoE}$ and LDLR only. AA-induced generation of $\mathrm{PGI}_{2}$ was comparable across strains, although $\mathrm{TxA}_{2}$ generation was increased in ApoE and LDLR. Arteriolar reactivity to $\mathrm{PGI}_{2}$ and $\mathrm{TXA}_{2}$ was comparable across strains. Treatment with TEMPOL improved dilation and reduced $\mathrm{TxA}_{2}$ production with $\mathrm{AA}$ in ApoE and LDLR.

Conclusions: These results suggest that AA-induced arteriolar dilation is constrained in ApoE and LDLR via an increased production of $\mathrm{TxA}_{2}$. While partially due to elevated oxidant stress, additional mechanisms contribute which are independent of acute alterations in oxidant tone. 


\section{INTRODUCTION}

Dyslipidemia, and specifically hypercholesterolemia, has repeatedly been demonstrated to represent a strong predisposing risk factor for the development of coronary and peripheral arterial disease (1). While this increased risk for the progression of vascular disease with hypercholesterolemia is most commonly associated with an increased predisposition for the development of atherothromboses, atherosclerotic lesions and plaque depositions $(3,10,26)$, investigations into the impact of hypercholesterolemia on vascular reactivity and endothelial function, potentially as contributing mechanisms to vascular disease, is less clearly understood.

While some disparity in the prevailing literature exists (25), the general consensus is that the development of hypercholesterolemia is usually associated with a significant reduction in the bioavailability of endothelium-derived nitric oxide $(5,6,23)$, with the relatively predictable ensuing outcome of an impaired vascular reactivity in response to stimuli that are considered to have a significant contribution from this signaling molecule/pathway (i.e., flow-mediated dilation; ref. 12). In our recent study, we provided evidence suggesting that development of familial hypercholesterolemia (a genetic disorder resulting in exceptionally high low density lipoprotein [LDL] level, in the face of an otherwise relatively normal lipid profile) in the LDL receptor gene deletion mouse or type III hyperlipidemia (a condition wherein both LDL and plasma triglycerides are significantly elevated) in the apolipoprotein E gene deletion mouse, was associated with a near complete abolition of the bioavailability of endothelium-derived nitric oxide in response to imposed stimuli (22). However, this loss of vascular nitric oxide bioavailability did not result in a profound reduction in dilator reactivity, as an increased generation of dilator signaling 
molecules through 12/15 lipoxygenases emerged with evolution of the dyslipidemia (22), suggesting that alterations to the metabolism of arachidonic acid may be associated with hypercholesterolemia, and that these can have profound consequences for vascular function.

In 1996, the work of Pfister and colleagues $(16,17)$ strongly suggested that dietinduced hypercholesterolemia in rabbits can lead to changes in arachidonic acid metabolism, mediated via lipoxyegnase are cytochrome P450 epoxygenase enzymes, causing profound alterations to dilator reactivity determined in isolated aortic segments. Additionally, Srisawat et al. (21), while providing additional evidence that diet-induced hypercholesterolemia results in impaired endothelium-dependent dilation in aortic rings, determined that chronic treatment with indomethacin improved endothelial function, and was associated with reductions in urinary levels of 2,3-dinor-thromboxane $B_{2}$ and 8-iso$\mathrm{PGF}_{2 \alpha}$, a stable urinary breakdown product of thromboxane $\mathrm{A}_{2}$ and a marker of chronic oxidant stress, respectively. Most recently, Pfister demonstrated that impairments to endothelium-dependent dilation in aortic rings of hypercholesterolemic rabbits were diminished in a subgroup of animals lacking a functional thromboxane receptor (15). These previous results suggest that a contributing mechanism underlying alterations to vascular reactivity under conditions of hypercholesterolemia may involve both elevated vascular oxidant stress and metabolism of arachidonic acid through cyclooxygenase pathways. However, given recent observations in our laboratory (22) and by others (25) suggesting that alterations to endothelium-dependent reactivity may reflect the specific challenge imposed rather than a global impairment, we examined alterations to dilator reactivity in response to direct challenge with arachidonic acid itself, wherein the 
bioavailability of endothelium-derived nitric oxide is not a significant contributing element to the net mechanical response. Using both apolipoprotein E and LDL receptor gene deletion mouse models of hypercholesterolemia, the hypothesis tested in the present study was that arachidonic acid-induced dilator reactivity of skeletal muscle arterioles would be impaired in the presence of profound dyslipidemia and that this would be the result of alterations to either the production or vascular reactivity to metabolites of arachidonic acid via cyclooxygenase, owing to the presence of an elevated oxidant stress.

\section{MATERIALS AND METHODS}

Animals: The present study used three strains of mice, the C57/BI/6J (C57) as the control strain and the apolipoprotein $\mathrm{E}$ gene deletion (B6.129P2-Apoe $\left.{ }^{t m 1 U n c} / \mathrm{J} ; \mathrm{ApoE}\right)$ and low density lipoprotein receptor gene deletion (B6.129S7-Ldlr ${ }^{\text {tm1Her} / J ; ~ L D L R) ~ m i c e ~ o n ~ t h e ~}$ C57/BI/6J background. All mice were purchased from Jackson Laboratories (Bar Harbor, ME) at 6 weeks of age. The ApoE mouse manifests type III hyperlipidemia, in which both plasma cholesterol and triglyceride levels are elevated, although the elevations in LDL are not as severe as in the LDLR gene deletion mouse (19). In contrast, the LDLR mouse is a model of human familial hypercholesterolemia, manifesting a profound increase in serum LDL levels while ingesting a normal diet (11).

Male mice of each strain were fed standard chow and drinking water ad libitum and were housed in an AAALAC-accredited animal care facility at the West Virginia University Health Sciences Center and all protocols received prior IACUC approval. At 20 weeks of age, after an overnight fast, mice were anesthetized with injections of sodium pentobarbital (50 mg.kg ${ }^{-1}$ i.p.), and received tracheal intubation to facilitate maintenance of a patent airway. In all mice, a carotid artery was cannulated for determination of arterial pressure. 
Blood aliquots were drawn from the jugular vein cannula for determination of glucose and insulin (Linco), a lipid profile (Waco), and nitrotyrosine (Oxis).

Preparation of Isolated Skeletal Muscle Resistance Arterioles: In anesthetized mice, the intramuscular continuation of the right gracilis artery was removed and cannulated, as described previously (8). These first order arterioles were extended to their approximate in situ length and were equilibrated at $80 \%$ of the animal's mean arterial pressure in order to approximate the in vivo intralumenal pressure experienced by the animal (13). Following equilibration, arteriolar reactivity was evaluated in response to increasing concentrations of arachidonic acid $\left(10^{-10} \mathrm{M}-10^{-6} \mathrm{M}\right.$; Sigma). Additionally, in select experiments arteriolar reactivity was also evaluated in response to increasing concentrations of prostacyclin $\left(\mathrm{PGI}_{2} ; 10^{-10} \mathrm{M}-10^{-6} \mathrm{M}\right.$; Biomol) or carbocyclic thromboxane $A_{2}\left(T_{x} A_{2} ; 10^{-10} \mathrm{M}-10^{-6} \mathrm{M}\right.$; Cayman).

Removal of the arteriolar endothelium was accomplished by passing an air bolus through the perfusate line into the isolated microvessel, the efficacy of which was determined from a loss of all dilator reactivity in response to application of $10^{-6} \mathrm{M}$ acetylcholine (8). To assess the contribution of nitric oxide production or the generation of metabolites via cyclooxygenase as mediators of arteriolar reactivity, isolated vessels were treated with the nitric oxide synthase inhibitor $L-N^{G}$-nitroarginine methyl ester (LNAME; $10^{-4} \mathrm{M}$ for 45 minutes prior to agonist challenge; Sigma) or the cyclooxygenase antagonist indomethacin (INDO; $10^{-6} \mathrm{M}$ for 60 minutes prior to agonist challenge; Sigma), respectively. To determine the contribution of metabolites of arachidonic acid mediated via cytochrome P450 enzymes, vessels were treated with the suicide substrate inhibitor 17-octadecynoic acid (17-ODYA; $10^{-5} \mathrm{M}$ for 60 minutes prior to 
agonist challenge; Sigma). Previous studies have demonstrated that 17-ODYA profoundly attenuates both the $\omega$-hydroxylation (producing 20-hydroxyeicosatetraenoic acid; 20-HETE) and epoxygenation (producing epoxyeicosatrienoic acids; EETs) reactions of arachidonic acid through cytochrome P450 (24), thus preventing changes to vascular levels of 20-HETE or EETs as contributing mediators to endotheliumdependent dilation. To assess the contribution of lipoxygenase metabolites to the patterns of arteriolar dilation, vessels were treated with nordihydroguaiaretic acid (NDGA; $3 \times 10^{-5} \mathrm{M}$ for 45 minutes prior to agonist challenge; Biomol), a selective inhibitor of 12/15-lipoxygenases $(20,27)$. To antagonize vascular $\mathrm{PGH}_{2} / \mathrm{T} x \mathrm{~A}_{2}$ receptors, vessels were treated with SQ-29548 (10 $0^{-5} \mathrm{M}$ for 30 minutes prior to agonist challenge; Biomol), while inhibition of thromboxane synthase was accomplished using carboxyheptyl imidazole $\left(\mathrm{CHI} ; 10^{-5} \mathrm{M}\right.$ for 45 minutes prior to agonist challenge; Biomol). To reduce vascular oxidant tone, arterioles were treated with 4-Hydroxy-2,2,6,6tetramethylpiperidine- $1-{ }^{15} \mathrm{~N}$-oxyl (TEMPOL; $10^{-4} \mathrm{M}$ for 60 minutes prior to agonist challenge, Sigma).

Determination of Vascular Metabolites of Arachidonic Acid: Vascular production of 6keto-prostaglandin $\mathrm{F}_{1 \alpha}$ (6-keto-PGF$F_{1 \alpha}$; the stable breakdown product of $P \mathrm{PI}_{2}$; ref. 14), and 11-dehydro-thromboxane $B_{2}$ (11-dehydro-TxB $B_{2}$; the stable plasma breakdown product of $\mathrm{TxA}_{2}$; ref. 4) in response to challenge with arachidonic acid within the three mouse strains was assessed using pooled conduit arteries (femoral, saphenous, iliac, carotid arteries) from each mouse. Vessels were incubated in microcentrifuge tubes in $1 \mathrm{ml}$ of physiological salt solution for 30 minutes under control conditions $\left(21 \% \mathrm{O}_{2}\right)$, after which time arachidonic acid $\left(10^{-6} \mathrm{M}\right)$ was added to the tube for an additional 30 minutes. After the second 30 
minute period, the PSS was transferred to a new tube, frozen in liquid $\mathrm{N}_{2}$ and stored at $80^{\circ} \mathrm{C}$. Metabolite release by the vessels was determined using commercially available EIA kits for 6-keto-PGF ${ }_{1 \alpha}$ and 11-dehydro-TxB 2 (Cayman).

Data and Statistical Analyses: Active tone of individual arterioles at the equilibration pressure was calculated as $\left(\Delta D / D_{\max }\right) \cdot 100$, where $\Delta D$ is the diameter increase from rest in response to $\mathrm{Ca}^{2+}$-free PSS, and $D_{\max }$ is the maximum diameter measured at the equilibration pressure in $\mathrm{Ca}^{2+}$-free PSS.

Dilator responses of isolated arterioles following challenge with dilator agonists were fit with the three-parameter logistic equation:

$$
y=\min +\left[\frac{\max -\min }{1+10^{\log E D_{50}-x}}\right]
$$

where $y$ represents the change in arteriolar diameter, "min" and "max" represent the lower and upper bounds, respectively, of the change in arteriolar diameter with increasing agonist concentration, $x$ is the logarithm of the agonist concentration and $\log E D_{50}$ represents the logarithm of the agonist concentration $(x)$ at which the response $(y)$ is halfway between the lower and upper bounds.

Data are presented as mean \pm SEM. Statistically significant differences in measured and calculated parameters in the present study were determined using analysis of variance (ANOVA). In all cases, Student-Newman-Keuls post hoc test was used when appropriate and $p<0.05$ was taken to reflect statistical significance.

\section{RESULTS}

Table 1 presents baseline characteristics of the mouse groups in the present study. While all mice were of similar mass at 20 weeks of age, LDLR experienced a significant 
elevation in mean arterial pressure and fasting insulin concentration versus values in C57 or ApoE. Additionally, both ApoE and LDLR manifested a profound hypercholesterolemia, most severe in LDLR. Further, ApoE exhibited a significant hypertriglyceridemia as well, while plasma triglyceride levels in LDLR were not different from that in C57. Finally, plasma levels of nitrotyrosine, a marker of chronic elevations in oxidant stress, were significantly elevated in ApoE and LDLR as compared to C57. With regard to basal vascular tone, isolated arterioles from all mouse groups demonstrated a comparable resting active diameter and passive (calcium-free) diameter, such that no significant difference in active tone was calculated between C57, ApoE and LDLR in the present study.

Data summarizing the dilator responses of skeletal muscle resistance arterioles from C57, ApoE and LDLR in response to challenge with increasing concentrations of arachidonic acid are presented in Figure 1. Under control conditions, the reactivity of arterioles from ApoE and LDLR, while not significantly different from each other, both demonstrated a reduction in their maximum bound as compared to responses in arterioles from C57. Endothelium-denudation via perfusion with an air bolus eliminated mechanical responses of vessels across the three strains in response to application of arachidonic acid.

The effects of pharmacological blockade of lipoxygenases and cyclooxygenases with NDGA and INDO, respectively, on arachidonic acid-induced vasodilation in isolated arterioles are summarized in Figure 2. In arterioles from C57 (Panel A), blockade of lipoxygenases with NDGA had no impact on dilator responses to arachidonic acid, while treatment with indomethacin abolished all dilation to arachidonic acid. Arterioles from 
ApoE, while demonstrating a blunted overall reactivity to arachidonic acid, also experienced a severe reduction in dilator reactivity following cyclooxygenase inhibition with indomethacin (Panel B). However, while treatment with NDGA alone did not impact arachidonic acid-induced dilation in vessels from ApoE, application of NDGA to vessels that had been treated with indomethacin eliminated the residual dilation in response to arachidonic acid that remained following cyclooxygenase inhibition alone. Finally, arterioles from LDLR appeared to demonstrate a dilator response to arachidonic acid challenge that was dependent on the production of metabolites generated via both lipoxygenases and cyclooxygenases, as antagonists to these pathways given in isolation resulted in modest reductions to the compromised level of reactivity, while treatment with both NDGA and indomethacin abolished all arachidonic acid-induced reactivity (Panel C). Treatment of isolated arterioles from C57, ApoE or LDLR with either L-NAME or 17-ODYA did not result in either significant or consistent effects of dilator responses following challenge with increasing concentrations of arachidonic acid (data not shown).

Figure 3 presents the effects of antagonizing thromboxane $\mathrm{A}_{2}$ generation (with $\mathrm{CHI}$ ) and action (with SQ-29548) on dilator responses of skeletal muscle arterioles in the present study. In control animals, application of either CHI or SQ-29548 had no impact on arteriolar dilation in response to increasing concentrations of arachidonic acid (Panel A). In contrast, arterioles from both ApoE (Panel B) and LDLR (Panel C) exhibited a significant improvement to their degree of arachidonic acid-induced dilation relative to untreated conditions following either inhibition of thromboxane synthase with $\mathrm{CHI}$ or blockade of the $\mathrm{PGH}_{2} / \mathrm{TXA}_{2}$ receptor (SQ-29548). 
Data describing the arachidonic acid-induced generation of the cyclooxygenase products $\mathrm{PGI}_{2}$ (estimated from levels of 6-keto-PGF ${ }_{1 \alpha}$ ) and $\mathrm{TxA}_{2}$ (estimated from levels of 11-dehydro $\mathrm{TxB}_{2}$ ) from pooled arteries of the three mouse groups in the present study are summarized in Figure 4. Following application of $10^{-6} \mathrm{M}$ arachidonic acid, arteries from C57, ApoE and LDLR all demonstrated a significant increase in $\mathrm{PGI}_{2}$ release, the degree of which was comparable between the three mouse strains (Panel A). In contrast, arachidonic acid-induced generation of $\mathrm{TxA}_{2}$, while statistically significant in arteries from C57, demonstrated a substantially more robust response in vessels from both ApoE and LDLR (Panel B). Pre-treatment of pooled vessels with either $\mathrm{CHI}$ or indomethacin severely attenuated all arachidonic acid-induced $\mathrm{TxA}_{2}$ generation in all three strains.

Arteriolar reactivity in response to challenge with prostacyclin (Panel A) or carbocyclic thromboxane $A_{2}$ (Panel $B$ ) in the three mouse groups is summarized in Figure 5. In response to increasing concentrations of prostacyclin, arterioles from C57 and ApoE demonstrated a very similar degree of dilator reactivity, although this response demonstrated a trend toward impairment in vessels from LDLR as compared to that in vessels from either other strain (Panel A). Arterioles from all three mouse strains exhibited very similar patterns of constrictor reactivity in response to challenge with increasing concentrations of carbocyclic thromboxane $A_{2}$ (Panel B).

Figure 6 presents the effects of treating vessels with the antioxidant TEMPOL, the thromboxane synthase inhibitor $\mathrm{CHI}$, or both, on arteriolar responses to increasing concentrations of arachidonic acid. Addition of TEMPOL did not have a significant impact on arteriolar diameter in vessels from any of the three mouse strains under resting conditions. In arterioles from C57 (Panel A), neither treatment with TEMPOL nor $\mathrm{CHI}$ had 
a significant impact on dilator reactivity to arachidonic acid. In contrast, for arterioles from both ApoE (Panel B) and LDLR (Panel C), treatment with either TEMPOL or $\mathrm{CHI}$ significantly improved dilator responses to arachidonic acid, with the effects of $\mathrm{CHI}$ being stronger than that for TEMPOL. Interestingly, in both ApoE and LDLR, combined treatment with $\mathrm{CHI}$ and TEMPOL did not have any effect on arachidonic acid-induced dilation beyond that determined for $\mathrm{CHI}$ treatment alone.

Figure 7 presents data describing the effects of treating arteries from C57, ApoE or LDLR with TEMPOL on arachidonic acid-induced thromboxane $A_{2}$ production. While treatment with the antioxidant had an insignificant impact on vascular thromboxane production in C57, incubation of vessels with TEMPOL significantly reduced the arachidonic acid-induced production of $\mathrm{TxA}_{2}$ in both ApoE and LDLR. However, this reduction in thromboxane generation was only partial in nature, and levels of $\mathrm{TxA}_{2}$ production in response to challenge with arachidonic acid following treatment with TEMPOL remained significantly increased versus that in untreated arteries from ApoE and LDLR.

\section{DISCUSSION}

Although hypercholesterolemia represents a powerful risk factor for the development of peripheral artery disease (1), the effects of hypercholesterolemia on vascular reactivity and endothelial function is less clearly understood. Given recent studies suggesting that diet-induced hypercholesterolemia can alter arachidonic acid metabolism and profoundly impact vascular reactivity through signaling mechanisms associated with the generation of thromboxane $A_{2}(15,18,21)$, the present study determined the effects of genetic hypercholesterolemia on the dilator reactivity of skeletal muscle resistance 
arterioles in response to challenge with arachidonic acid. More specifically, the hypothesis tested in this study was that arachidonic acid-induced arteriolar dilation in ApoE and LDLR would be impaired owing to either the production of, or vascular reactivity to, metabolites of arachidonic acid via cyclooxygenase, and that these alterations would be associated with an elevated oxidant stress.

Contrary to our results with dilator stimuli that are more strongly dependent on the bioavailability of endothelium-derived nitric oxide, where reactivity was largely maintained in the face of a profound reduction in this parameter (22), the results presented in Figure 1 indicate that skeletal muscle arteriolar dilation in response to increasing concentrations of arachidonic acid was significantly reduced in both ApoE and LDLR as compared to responses determined in C57. Interestingly, the data presented in this figure also strongly suggest that not only is the overwhelming majority of dilator reactivity in response to arachidonic acid dependent on a functional endothelium in control animals, the impairments to arteriolar dilation with this stimulus may also originate with alterations to endothelial function, rather than within vascular smooth muscle.

While results from the present study did not demonstrate a role for either nitric oxide bioavailability or for metabolites of arachidonic acid mediated via cytochrome P450 enzymes in terms of contributing to the arachidonic acid-induced dilator reactivity of skeletal muscle arterioles in any of the three mouse strains, activity mediated through cyclooxygenase (and to a lesser extent lipoxygenase) were critical. While arteriolar dilation in response to arachidonic acid was mediated entirely via cyclooxygenase in vessels from C57, vessels from ApoE and LDLR demonstrated a dilator response that was increasingly a function of metabolites via both cyclooxygenase and lipoxygenase, with this effect being 
more pronounced in LDLR than in ApoE, where the response was still predominantly cyclooxygenase-dependent. However, the data presented in Figure 2 do not provide significant insight into the impaired dilator reactivity demonstrated in arterioles of ApoE and LDLR in response to challenge with arachidonic acid beyond the critical involvement of cyclooxygenase. Given previous studies suggesting that the development of the hypercholesterolemic condition can profoundly impact arachidonic acid metabolism in general $(7,18)$, and the recent studies from both Pfister (15) and Srisawat et al. (21) that implicate altered behavior mediated through thromboxane generation/action as contributing mechanism to altered patterns of vascular reactivity with hypercholesterolemia, we treated vessels from ApoE and LDLR with an inhibitor of thromboxane synthase $(\mathrm{CHI})$ or an antagonist for the $\mathrm{PGH}_{2} / \mathrm{TxA}_{2}$ receptor (SQ-29548). As shown in Figure 3, while neither of these agents had a significant role in the dilator responses in arterioles from C57, application of either $\mathrm{CHI}$ or SQ-29548 resulted in a significant improvement in the dilator responses of arterioles from ApoE or LDLR in response to challenge with increasing concentrations of arachidonic acid. Interestingly, the ameliorative effect was comparable with either pharmacological agent. While this implicates either increased thromboxane generation or an increased gain/sensitivity at the vascular thromboxane receptor as contributing mechanisms to the impaired arachidonic acid-induced arteriolar dilation, these data do not provide insight into which component may be most responsible. However, these data do strongly suggest that the development of a thromboxane-sensitive component which may act to constrain arachidonic acid-induced arteriolar dilation accompanies the evolution of genetic hypercholesterolemia. 
As both $\mathrm{CHI}$ and SQ-29548 elicited similar improvements to arteriolar dilation in response to arachidonic acid challenge in ApoE and LDLR, it was necessary to discern which processes contributed to the constrained dilator reactivity: 1) increasing thromboxane $A_{2}$ production in response to arachidonic acid production, 2) increased vascular reactivity to produced thromboxane $A_{2}$, or both. The data presented in Figure 4 indicate that arachidonic acid-induced generation of $\mathrm{PGI}_{2}$ (estimated from 6-keto-PGF $\mathrm{P}_{1 \alpha}$ levels) remained intact in arteries of ApoE and LDLR as compared to that determined in C57, an observation that is consistent with previous studies in the coronary vasculature of ApoE mice (9). In contrast, arachidonic-acid induced generation of thromboxane $A_{2}$ (estimated from 11-dehydro- $\mathrm{TxB}_{2}$ levels) was significantly increased with the evolution of genetic hypercholesterolemia in ApoE and LDLR. When taken together with data in Figure 5 , which suggest that the sensitivity of resistance arterioles from ApoE and LDLR in response to increasing concentrations of either prostacyclin or thromboxane $A_{2}$ is not dramatically altered from that determined for C57 control mice, these data may provide compelling evidence that a predominant contributing mechanism underlying the constrained arteriolar dilation with increasing concentrations of arachidonic acid may be the development of an increased generation of the constrictor prostanoid thromboxane $A_{2}$, which antagonizes the dilator effects associated with the generation of prostacyclin.

Given that previous studies have clearly demonstrated the critical role for elevated oxidant tone in the increased generation of thromboxane through cyclooxygenase in response to challenge with arachidonic acid $(2,28,29)$, and our observations of an increase in the plasma levels of nitrotyrosine in the ApoE and LDLR as compared to that determined in C57, the data presented in Figures 6 and 7 provide some insight into the 
potential role that elevated vascular oxidant tone may play in the increased arachidonic acid-induced thromboxane $A_{2}$ generation with genetic dyslipidemia. While treatment with TEMPOL had no impact on arachidonic acid-induced dilation or $\mathrm{TXA}_{2}$ generation in arterioles from C57, it significantly improved the dilator response in microvessels from both ApoE and LDLR and reduced the levels of $\mathrm{TxA}_{2}$ production. However, in vessels from both strains, this improvement in dilator reactivity following treatment with the antioxidant was less pronounced than that determined following treatment with the inhibitor of thromboxane synthase, $\mathrm{CHI}$. Further, combined treatment with both TEMPOL and $\mathrm{CHI}$ did not result in an improvement beyond that determined with $\mathrm{CHI}$ treatment alone. Additionally, while pretreatment of pooled vessels with TEMPOL lowered arterial thromboxane production in response to challenge with arachidonic acid, the levels of thromboxane production remained significantly elevated despite the addition of the antioxidant. Taken together these results suggest that, while an enhanced arachidonic acid-induced genesis of thromboxane $A_{2}$ via thromboxane synthase represents a strong contributor to the constrained dilator reactivity in skeletal muscle arterioles of ApoE and LDLR mice, the presence of an elevated vascular oxidant tone may represent a partial contributor to this shift in the metabolism of arachidonic acid. Clearly, these results suggest that other parameters, independent of acute changes in vascular oxidant tone, also contribute to this increased generation of thromboxane $A_{2}$. Potential avenues for ongoing investigation in this regard can include the study of not only the effects of chronic elevations in vascular oxidant tone, but also the progression of a chronic state of inflammation associated with dyslipidemia $(10,26)$ and how these processes can ultimately impact pathways of arachidonic acid metabolism. 
In summary, with the development of genetic hypercholesterolemia in ApoE and LDLR mice, the dilator reactivity of skeletal muscle resistance arterioles in response to increasing concentrations of arachidonic acid is impaired. This impairment does not appear to be associated with a reduction in the generation/release of, or an altered arteriolar reactivity to, prostacyclin. However, with the evolution of this dyslipidemic condition, there appears to be an increase in the arachidonic acid-induced generation of the vasoconstrictor metabolite thromboxane $A_{2}$. While there does not appear to be an alteration to the arteriolar constrictor reactivity to thromboxane, the increased generation of this metabolite may compete with the dilator effects of prostacyclin, thus limiting net dilator reactivity in response to arachidonic acid. Further, while an increase in vascular oxidant stress appears to contribute to this response, additional mechanisms which are independent of acute alterations to oxidant tone also contribute to this effect. Future investigation will be required to discern which mechanistic alterations associated with the development of hypercholesterolemia contribute to the increased production of thromboxane $A_{2}$, and what the implications of this shift in the metabolism of arachidonic acid are for issues such as the integrated control of tissue perfusion, tissue oxygenation and the protection from atherogenesis and atherothrombosis. 


\section{ACKNOWLEDGEMENTS}

The authors gratefully acknowledge the support provided through the Translational

Research Core in the Center for Interdisciplinary Research in Cardiovascular Sciences at the West Virginia University Health Sciences Center in the performance of this study. 
Reference List

1. American Heart Association Statistical Summary Sheets; "High Blood Cholesterol and Other Lipids": http://www.americanheart.org/presenter.jhtml?identifier=3000945, 2007.

2. Bachschmid M, Thurau S, Zou MH, Ullrich V. Endothelial cell activation by endotoxin involves superoxide/NO-mediated nitration of prostacyclin synthase and thromboxane receptor stimulation. FASEB J. 2003; 17:914-6.

3. Bhatt DL, Steg PG, Ohman EM, Hirsch AT, Ikeda Y, Mas JL, Goto S, Liau CS, Richard AJ, Rother J, Wilson PW; REACH Registry Investigators. International prevalence, recognition, and treatment of cardiovascular risk factors in outpatients with atherothrombosis. JAMA. 2006; 295:180-9.

4. Catella F, Healy D, Lawson JA, FitzGerald GA. 11-Dehydrothromboxane $B_{2}$ : a quantitative index of thromboxane $\mathrm{A}_{2}$ formation in the human circulation. Proc Natl Acad Sci U S A. 1986; 83:5861-5.

5. de Jongh S, Lilien MR, op't Roodt J, Stroes ES, Bakker HD, Kastelein JJ. Early statin therapy restores endothelial function in children with familial hypercholesterolemia. J Am Coll Cardiol. 2002; 40:2117-21. 
6. Engler MM, Engler MB, Malloy MJ, Chiu EY, Schloetter MC, Paul SM, Stuehlinger M, Lin KY, Cooke JP, Morrow JD, Ridker PM, Rifai N, Miller E, Witztum JL, MietusSnyder M. Antioxidant vitamins $\mathrm{C}$ and $\mathrm{E}$ improve endothelial function in children with hyperlipidemia: Endothelial Assessment of Risk from Lipids in Youth (EARLY) Trial. Circulation. 2003; 108:1059-63.

7. Feletou M, Vanhoutte PM. Endothelial dysfunction: a multifaceted disorder (The Wiggers Award Lecture). Am J Physiol Heart Circ Physiol. 2006; 291:H985-1002.

8. Frisbee JC, Maier KG, Falck JR, Roman RJ, Lombard JH. Integration of hypoxic dilation signaling pathways for skeletal muscle resistance arteries. Am J Physiol Regul Integr Comp Physiol. 2002; 283:R309-19.

9. Godecke A, Ziegler M, Ding Z, Schrader J. Endothelial dysfunction of coronary resistance vessels in apoE-/- mice involves NO but not prostacyclin-dependent mechanisms. Cardiovasc Res. 2002; 53:253-62.

10. Hansson GK. Inflammation, atherosclerosis, and coronary artery disease. N Engl J Med. 2005; 352:1685-95.

11. Ishibashi S, Brown MS, Goldstein JL, Gerard RD, Hammer RE, Herz J. Hypercholesterolemia in low density lipoprotein receptor knockout mice and its reversal by adenovirus-mediated gene delivery. J Clin Invest. 1993; 92:883-93. 
12. Joyner MJ, Dietz NM. Nitric oxide and vasodilation in human limbs. J Appl Physiol. $1997 ; 83: 1785-96$.

13. Liu Y, Fredricks KT, Roman RJ, Lombard JH. Response of resistance arteries to reduced PO2 and vasodilators during hypertension and elevated salt intake. Am J Physiol. 1997; 273:H869-77.

14. Nies AS. Prostaglandins and the control of the circulation. Clin Pharmacol Ther. 1986; 39:481-8.

15.Pfister SL. Aortic thromboxane receptor deficiency alters vascular reactivity in cholesterol-fed rabbits. Atherosclerosis. 2006; 189:358-63.

16. Pfister SL, Campbell WB. Contribution of arachidonic acid metabolites to reduced norepinephrine-induced contractions in hypercholesterolemic rabbit aortas. $J$ Cardiovasc Pharmacol. 1996; 28:784-91.

17.Pfister SL, Spitzbarth N, Edgemond W, Campbell WB. Vasorelaxation by an endothelium-derived metabolite of arachidonic acid. Am J Physiol. 1996; 270:H102130. 
18. Pfister SL, Falck JR, Campbell WB. Enhanced synthesis of epoxyeicosatrienoic acids by cholesterol-fed rabbit aorta. Am J Physiol. 1991; 261:H843-52.

19. Piedrahita JA, Zhang SH, Hagaman JR, Oliver PM, Maeda N. Generation of mice carrying a mutant apolipoprotein $\mathrm{E}$ gene inactivated by gene targeting in embryonic stem cells. Proc Natl Acad Sci U S A. 1992; 89:4471-5.

20. Salari $H$, Braquet $P$, Borgeat $P$. Comparative effects of indomethacin, acetylenic acids, 15-HETE, nordihydroguaiaretic acid and BW755C on the metabolism of arachidonic acid in human leukocytes and platelets. Prostaglandins Leukot Med. $1984 ; 13: 53-60$.

21. Srisawat S, Phivthong-Ngam L, Unchern S, Chantharaksri U, Govitrapong P, Sanvarinda Y. Improvement of vascular function by chronic administration of a cyclo-oxygenase inhibitor in cholesterol-fed rabbits. Clin Exp Pharmacol Physiol. $2003 ; 30: 405-12$.

22. Stapleton PA, Goodwill AG, James ME, Frisbee JC. Altered mechanisms of endothelium-dependent dilation in skeletal muscle arterioles with genetic hypercholesterolemia. Am J Physiol Regul Integr Comp Physiol. 293:R1110-R1119, 2007. 
23. Stokes KY, Cooper D, Tailor A, Granger DN. Hypercholesterolemia promotes inflammation and microvascular dysfunction: role of nitric oxide and superoxide. Free Radic Biol Med. 2002; 33:1026-36.

24. Wang MH, Brand-Schieber E, Zand BA, Nguyen X, Falck JR, Balu N, Schwartzman ML. Cytochrome P450-derived arachidonic acid metabolism in the rat kidney: characterization of selective inhibitors. J Pharmacol Exp Ther. 1998; 284:966-73.

25. Wolfle SE, de Wit C. Intact endothelium-dependent dilation and conducted responses in resistance vessels of hypercholesterolemic mice in vivo. $J$ Vasc Res. $2005 ; 42: 475-82$.

26. Zadelaar S, Kleemann R, Verschuren L, de Vries-Van der Weij J, van der Hoorn J, Princen HM, Kooistra T. Mouse models for atherosclerosis and pharmaceutical modifiers. Arterioscler Thromb Vasc Biol. 2007; 27:1706-21.

27.Zhang DX, Gauthier KM, Chawengsub Y, Holmes BB, Campbell WB. Cyclooxygenase- and lipoxygenase-dependent relaxation to arachidonic acid in rabbit small mesenteric arteries. Am J Physiol Heart Circ Physiol. 2005; 288:H302-9.

28.Zou MH, Cohen R, Ullrich V. Peroxynitrite and vascular endothelial dysfunction in diabetes mellitus. Endothelium. 2004; 11:89-97. 
29.Zou MH, Leist $M$, Ullrich $V$. Selective nitration of prostacyclin synthase and defective vasorelaxation in atherosclerotic bovine coronary arteries. Am J Pathol. 1999; 154:1359-65. 
Table 1. Baseline characteristics of mice and individual arterioles used in the present study. ${ }^{*} p<0.05$ vs. C57; $\uparrow p<0.05$ vs. ApoE.

\begin{tabular}{lccc}
\hline & C57 & ApoE & LDLR \\
\hline Mass $(\mathrm{g})$ & $33 \pm 2$ & $34 \pm 2$ & $33 \pm 2$ \\
MAP $(\mathrm{mmHg})$ & $88 \pm 4$ & $92 \pm 3$ & $106 \pm 5^{*} \dagger$ \\
{$[\text { Glucose }]_{\text {blood }}(\mathrm{mg} / \mathrm{dl})$} & $84 \pm 7$ & $103 \pm 11$ & $115 \pm 7^{*}$ \\
{$[\text { Insulin }]_{\text {plasma }}(\mathrm{ng} / \mathrm{ml})$} & $1.1 \pm 0.3$ & $1.6 \pm 0.3$ & $2.8 \pm 0.5^{*}$ \\
{$[\text { Total Cholesterol }]_{\text {plasma }}(\mathrm{mg} / \mathrm{dl})$} & $88 \pm 9$ & $288 \pm 17^{*}$ & $364 \pm 22^{*} \dagger$ \\
{$[\text { LDL Cholesterol }]_{\text {plasma }}(\mathrm{mg} / \mathrm{dl})$} & $49 \pm 5$ & $260 \pm 11^{*}$ & $338 \pm 19^{*} \dagger$ \\
{$[\text { Triglycerides }]_{\text {plasma }}(\mathrm{mg} / \mathrm{dl})$} & $88 \pm 10$ & $175 \pm 14^{*}$ & $116 \pm 18 \dagger$ \\
Nitrotyrosine \\
plasma
\end{tabular}




\section{FIGURE LEGENDS}

Figure 1. Data describing the dilator reactivity of isolated skeletal muscle resistance arterioles of C57, ApoE and LDLR mice in response to increasing concentrations of arachidonic acid. Data, presented as mean \pm SEM, are shown for arterioles under control conditions and following removal of the vascular endothelium using air bolus perfusion (please see text for details). $n=6$ animals for each strain; ${ }^{*} p<0.05$ vs. $C 57 ; \dagger p<0.05$ vs. control within that strain.

Figure 2. Data describing the dilator responses of isolated skeletal muscle resistance arterioles of C57 (Panel A), ApoE (Panel B) and LDLR (Panel C) mice in response to increasing concentrations of arachidonic acid. Data, presented as mean $\pm S E M$, are shown for arterioles under control conditions, and following pharmacological inhibition of cyclooxygenases with indomethacin, lipoxygenases with NDGA or combined inhibition of both enzymatic pathways (please see text for details). $n=5-10$ animals for each group; * $p<0.05$ vs. control conditions, $\uparrow p<0.05$ vs. no response.

Figure 3. Data describing the dilator responses of isolated skeletal muscle resistance arterioles of C57 (Panel A), ApoE (Panel B) and LDLR (Panel C) mice in response to increasing concentrations of arachidonic acid. Data, presented as mean $\pm S E M$, are shown for arterioles under control conditions, and following pharmacological inhibition of $\mathrm{PGH}_{2} / \mathrm{TxA}_{2}$ receptors with SQ-29548 and thromboxane synthase with $\mathrm{CHI}$ (please see text for details). $n=6-7$ animals for each group; * $p<0.05$ vs. control conditions. 
Figure 4. Data describing the arterial production of prostacyclin (as 6-keto-PGF $F_{1 \alpha}$; Panel A) or thromboxane $A_{2}$ (as 11-dehydro $\mathrm{TxB}_{2}$; Panel $\mathrm{B}$ ) from $\mathrm{C} 57$, ApoE and LDLR in response to $10^{-6} \mathrm{M}$ arachidonic acid. Data, presented as mean $\pm \mathrm{SEM}$, are shown under control conditions, and following pharmacological inhibition of cyclooxygenase with indomethacin or thromboxane synthase (with $\mathrm{CHI}$ ), as appropriate. $\mathrm{n}=8$ animals for each group, with each $\mathrm{n}$ representing pooled arteries from an individual mouse; please see text for details. ${ }^{*} p<0.05$ vs. respective control; $\uparrow p<0.05$ vs. C57 under that condition; $\ddagger$ vs. ApoE under that condition.

Figure 5. Data (mean \pm SEM) describing the reactivity of isolated skeletal muscle resistance arterioles of $\mathrm{C} 57, \mathrm{ApoE}$ and LDLR mice in response to increasing concentrations of prostacyclin (Panel A) or carbocyclic thromboxane $A_{2}$ (Panel B). $n=6$ animals for each group, no significant differences were identified in the vascular reactivity in response to increasing concentrations of prostacyclin or thromboxane $A_{2}$.

Figure 6. Data, presented as mean $\pm S E M$, describing the dilator responses of isolated skeletal muscle resistance arterioles of C57 (Panel A), ApoE (Panel B) and LDLR (Panel C) mice in response to increasing concentrations of arachidonic acid. Data are shown for arterioles under control conditions, following treatment of vessels with the antioxidant TEMPOL, following pharmacological inhibition of thromboxane synthase with $\mathrm{CHI}$, and following treatment with both TEMPOL and $\mathrm{CHI} . \mathrm{n}=8-10$ animals for each group; * $p<0.05$ vs. control conditions; $\uparrow p<0.05$ vs. treatment with TEMPOL alone. 
Figure 7. Data describing the arterial production of thromboxane $A_{2}$ (as 11-dehydro $T_{x B}$; Panel B) from C57, ApoE and LDLR in response to $10^{-6} \mathrm{M}$ arachidonic acid. Data, presented as mean \pm SEM, are shown under control conditions, and following treatment of pooled arteries with the antioxidant TEMPOL $\left(10^{-4} \mathrm{M}\right) . n=6$ animals for each group, with each $\mathrm{n}$ representing pooled arteries from an individual mouse; please see text for details. * $p<0.05$ vs. within-strain/no arachidonic acid; $\dagger p<0.05$ vs. within-strain/with arachidonic acid. 


\section{FIGURES}

\section{Figure 1.}

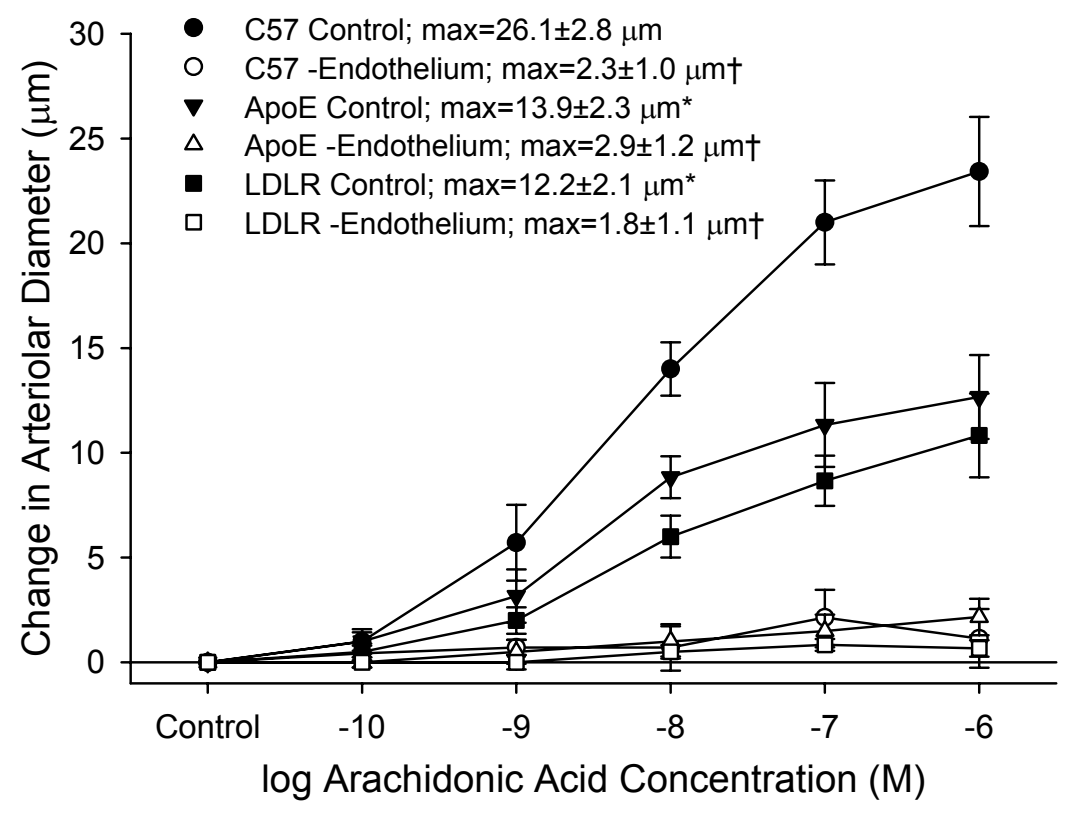


Figure 2.
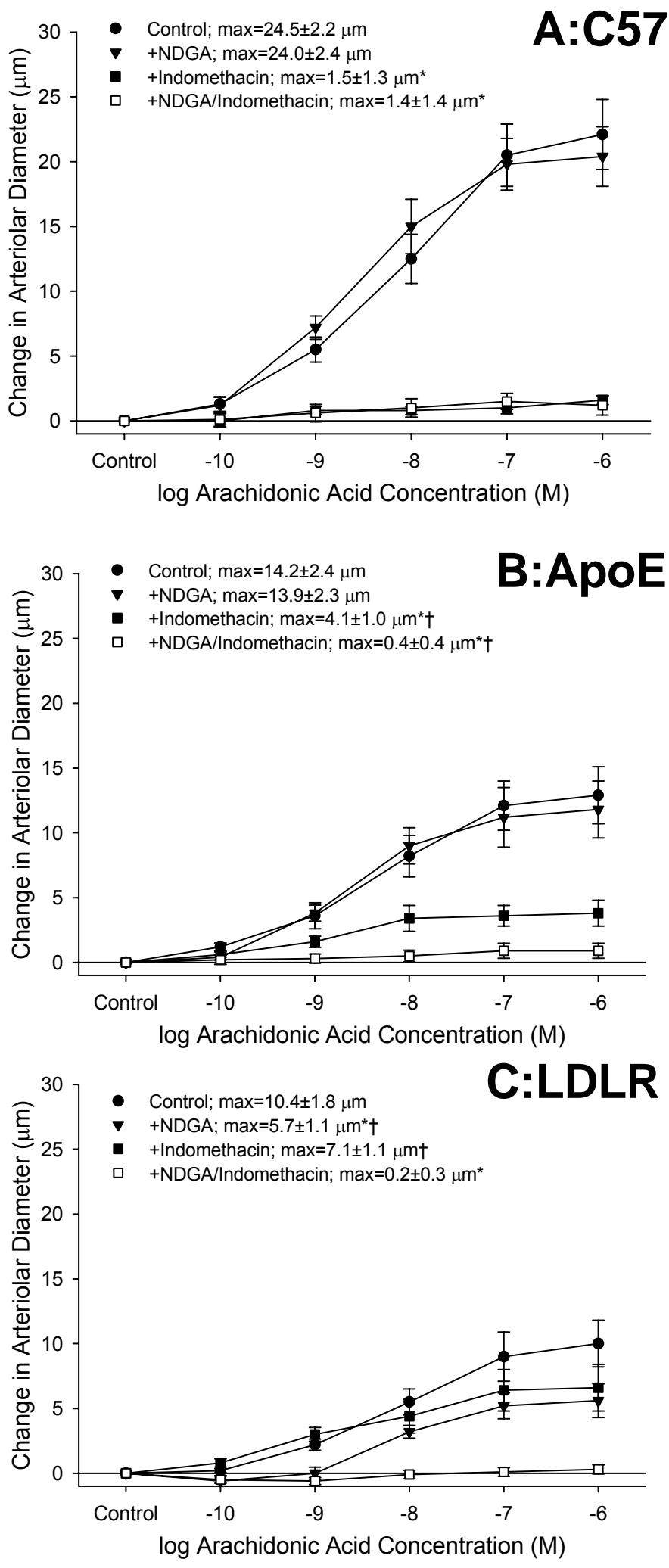
Figure 3.
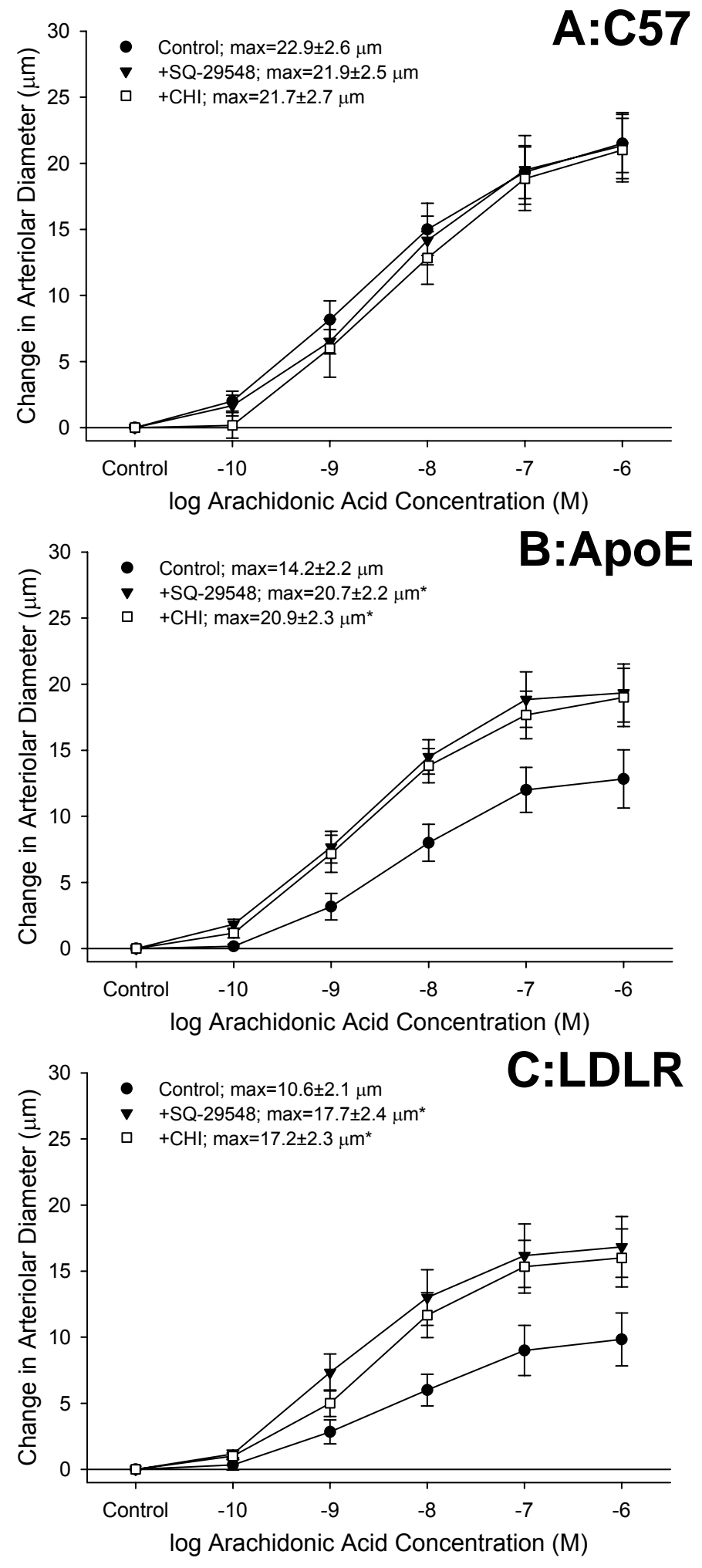
Figure 4.
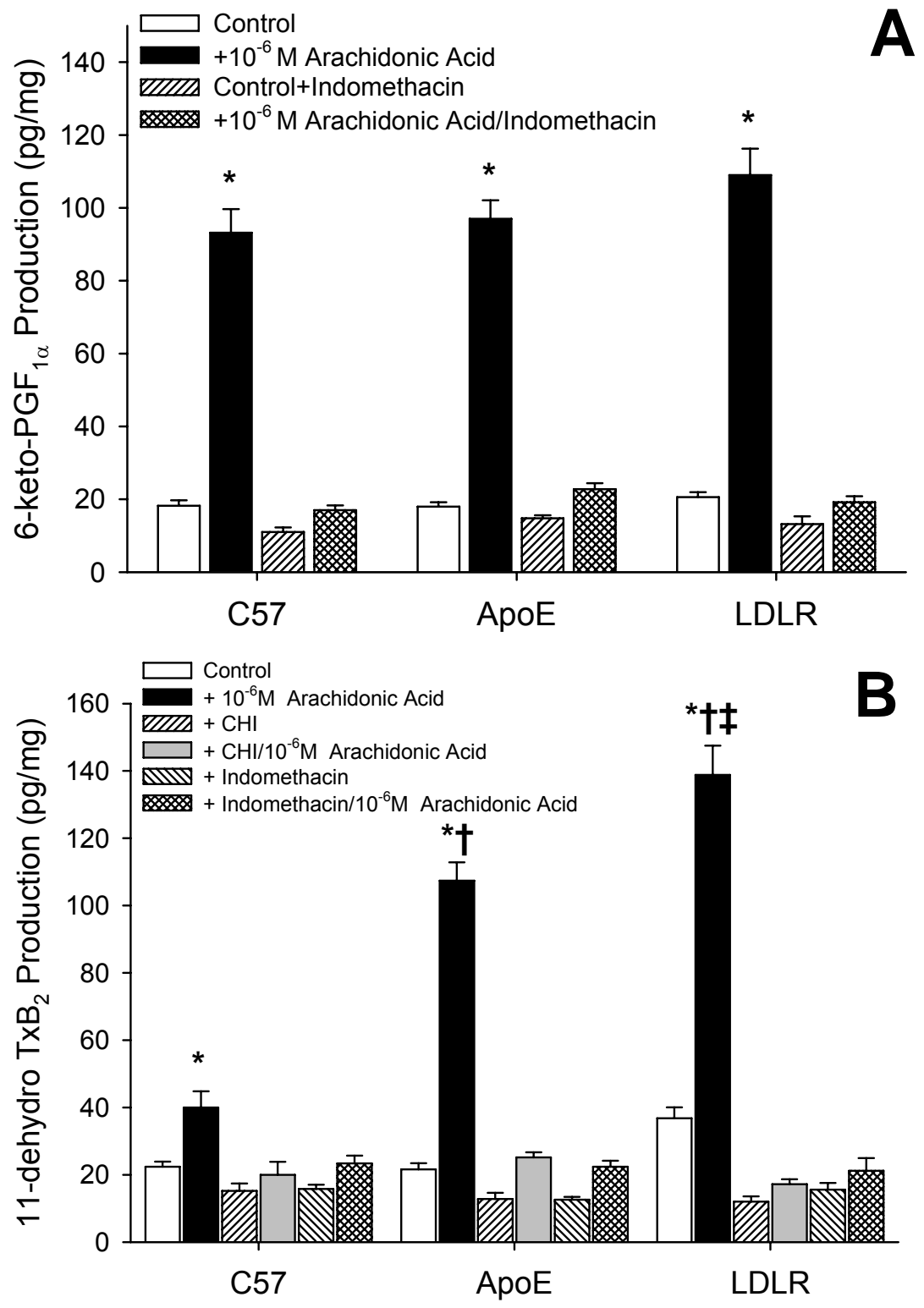
Figure 5.
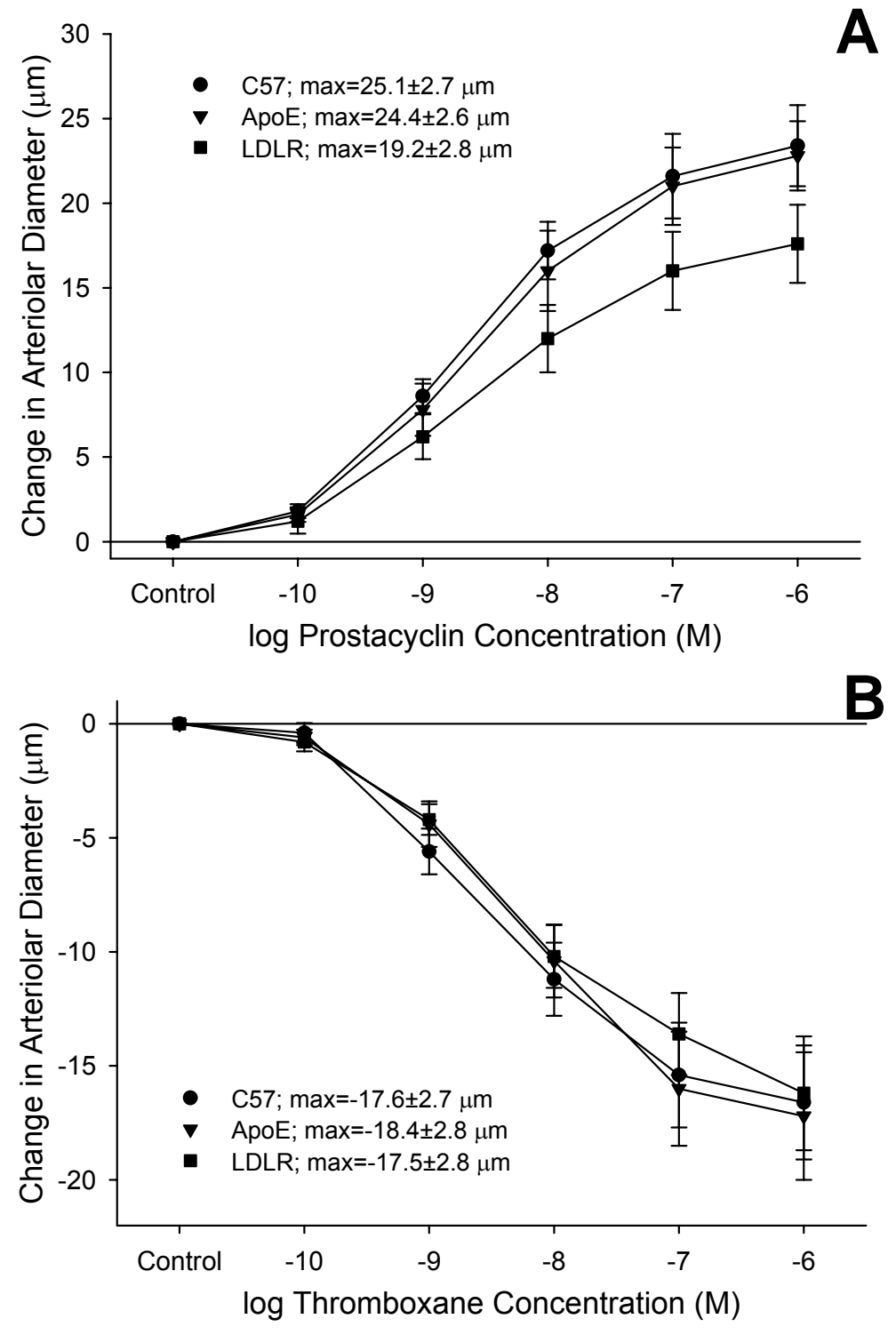
Figure 6.
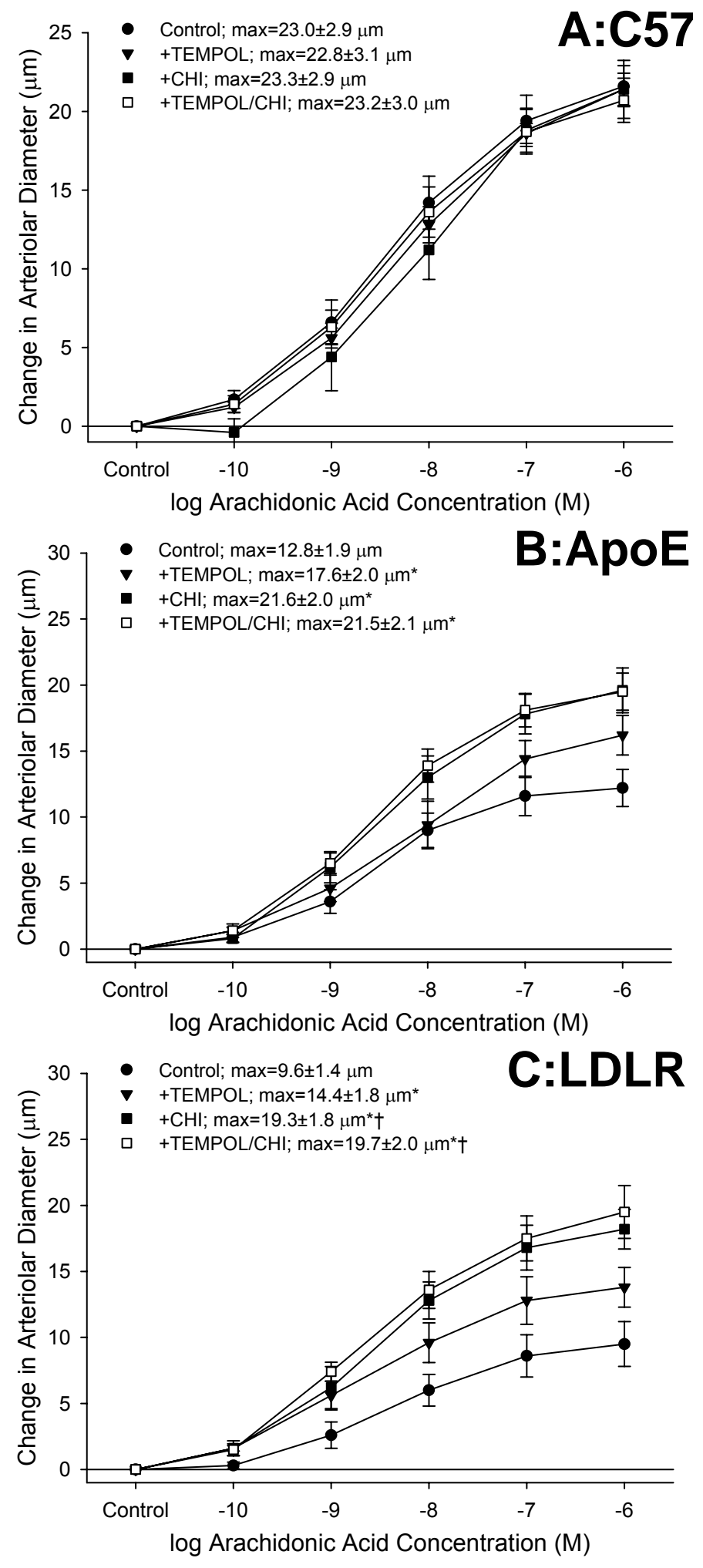
Figure 7.

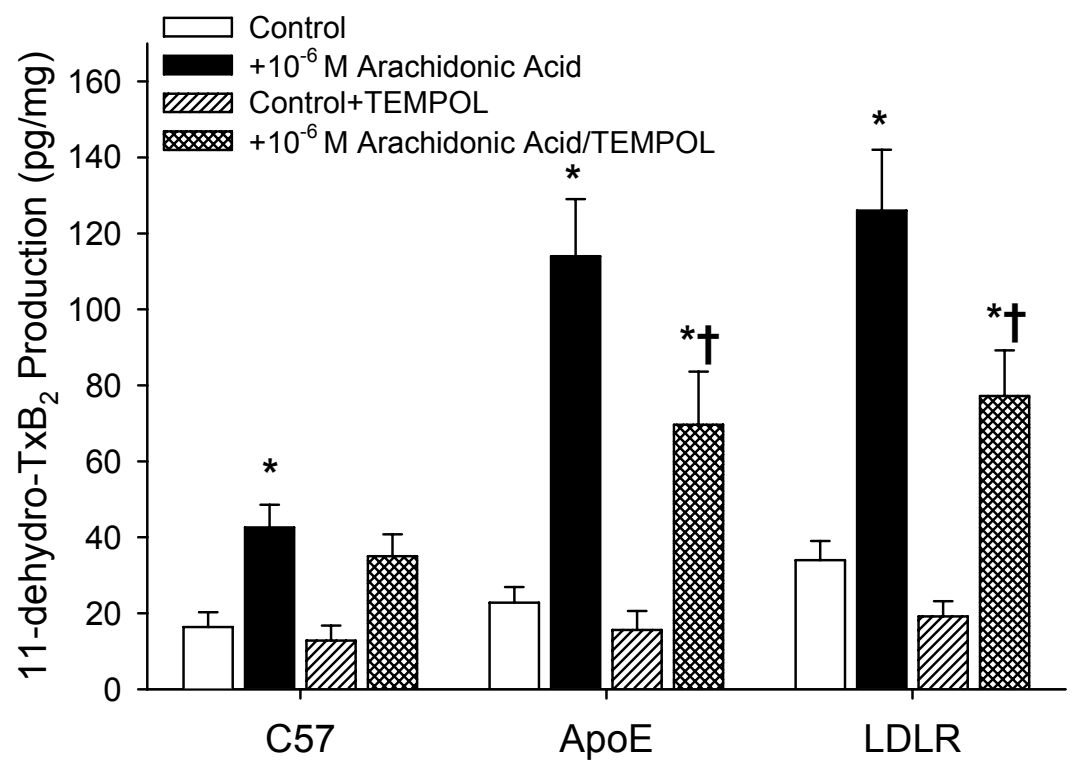




\title{
Chapter 4
}

\section{IMPACT OF CHRONIC ANTI-CHOLESTEROL THERAPY ON DEVELOPMENT OF MICROVASCULAR}

\section{RAREFACTION IN THE METABOLIC SYNDROME}

\author{
Adam G. Goodwill ${ }^{1,2}$, Stephanie J. Frisbee ${ }^{1,3}$, Phoebe A. Stapleton ${ }^{1,4}$, Milinda E. \\ James $^{1,2}$ \\ and Jefferson C. Frisbee ${ }^{1,2}$
}

\begin{abstract}
Center for Cardiovascular and Respiratory Sciences ${ }^{1}$
Departments of Physiology and Pharmacology ${ }^{2}$, Community Medicine ${ }^{3}$, and Exercise Physiology 4

West Virginia University School of Medicine, Morgantown, WV 26506
\end{abstract}

Running Head: Microvascular outcomes in obesity

Key Words: regulation of skeletal muscle perfusion, vascular remodeling, vascular reactivity, rodent models of obesity, nitric oxide bioavailability, chronic inflammation

Support: American Heart Association (SDG 0330194N; EIA 0740129N) and NIH (R01 DK64668).

"This research was originally published in Microcirculation. Goodwill AG, Frisbee SJ, Stapleton PA, James ME, Frisbee JC . Impact of chronic anticholesterol therapy on development of microvascular rarefaction in the metabolic syndrome. Microcirculation. 2009 Nov;16(8):667-84." 


\section{ABSTRACT}

Object: The obese Zucker rat (OZR) model of the metabolic syndrome is partly characterized by moderate hypercholesterolemia in addition to other contributing comorbidities. Previous results suggest that vascular dysfunction in OZR is associated with chronic reduction in vascular nitric oxide (NO) bioavailability and chronic inflammation, both frequently associated with hypercholesterolemia. As such, we evaluated the impact of chronic cholesterol reducing therapy on the development of impaired skeletal muscle arteriolar reactivity and microvessel density in OZR and its impact on chronic inflammation and NO bioavailability. Materials and Methods: Beginning at 7 weeks of age, male OZR were treated with gemfibrozil, probucol, atorvastatin or simvastatin (in chow) for 10 weeks. Subsequently, plasma and vascular samples were collected for biochemical/molecular analyses, while arteriolar reactivity and microvessel network structure were assessed using established methodologies after 3, 6 and 10 weeks of drug therapy. Results: All interventions were equally effective at reducing total cholesterol, although only the statins also blunted the progressive reductions to vascular NO bioavailability, evidenced by greater maintenance of acetylcholine-induced dilator responses, an attenuation of adrenergic constrictor reactivity, and an improvement in agonist-induced NO production. Comparably, while minimal improvements to arteriolar wall mechanics were identified with any of the interventions, chronic statin treatment reduced the rate of microvessel rarefaction in OZR. Associated with these improvements was a striking statin-induced reduction in inflammation in OZR, such that numerous markers of inflammation were correlated with improved microvascular reactivity and density. However, using 
multivariate discriminant analyses, plasma RANTES, IL-10, MCP-1 and TNF- $\alpha$ were determined to be the strongest contributors to differences between groups, although their relative importance varied with time. Conclusions: While the positive impact of chronic statin treatment on vascular outcomes in the metabolic syndrome are independent of changes to total cholesterol, and are more strongly associated with improvements to vascular NO bioavailability and attenuated inflammation, these results provide both a spatial and temporal framework for targeted investigation into mechanistic determinants of vasculopathy in the metabolic syndrome. 


\section{INTRODUCTION}

Arising from a chronic hyperphagia which originates due to non-functional leptin receptor gene and an impaired satiety reflex $(5,23)$, the obese Zucker rat $(\mathrm{OZR})$ rapidly develops insulin resistance, hypertriglyceridemia and a moderate hypertension (43). Combined with the parallel creation of a pro-thrombotic, pro-oxidant and pro-inflammatory environment, OZR are considered to be an excellent model for the clinical condition termed the metabolic syndrome (47). Associated with these systemic pathologies, we and others have defined numerous impairments to microvascular structure and function in OZR which negatively impact skeletal muscle perfusion, both under resting conditions $(15,18,19)$, in response to elevated metabolic demand $(18,19,50,51)$, following recovery from vascular occlusion (15) and during hemorrhage (16). Chronic treatment of the metabolic syndrome with exercise $(13,52)$ or of individual contributing elements through pharmacological intervention $(10,16,17,48)$ have resulted in improvements to microvascular outcomes, as well as to perfusion responses within skeletal muscle $(13,52)$, and have implicated potential mechanisms through which these improvements may be manifested. Recently, results from our laboratory have suggested that the chronic reduction in vascular nitric oxide (NO) bioavailability that accompanies development of the metabolic syndrome in OZR is well correlated with the severity of the reduction in skeletal muscle microvessel density (16). Ongoing studies have also suggested that while this microvascular rarefaction is hypertension-independent (17), exercise-based interventions that not only increase vascular NO bioavailability, but also blunt the severity of the chronic inflammatory state in OZR, may be an excellent predictor of the ability to prevent microvessel loss within the periphery (13). 
Within the metabolic syndrome in OZR is an elevation in plasma cholesterol levels that, while consistent, is more moderate as compared to the profound elevations determined for plasma triglycerides. Given the severity of many indices of vascular dysfunction in OZR, the extent to which elevated plasma cholesterol contributes to these impairments in currently unclear. However, treatment of hypercholesterolemia with 3hydroxy-3-methylglutaryl coenzyme A (HMG Co-A) reductase inhibitors ("statins") has not only the well-documented impact of lowering circulating plasma LDL and total cholesterol levels $(21,35)$, but also has been identified as having the beneficial impacts of increasing vascular NO bioavailability $(3,34)$ and blunting plasma markers of chronic inflammation $(11,32,35)$. Notably, it has been suggested that improvements to vascular function in human subjects or animals afflicted with hypercholesterolemia may reflect these pleiotropic effects of statin therapy rather than the direct impact of anti-cholesterol therapy itself $(3,26$, 31). However, the extent to which these reflect independent ameliorative effects or effects which are strongly correlated remains unclear.

The purpose of the present study was to determine the impact of chronic anticholesterol therapy on the temporal development of impairments to microvascular reactivity and network structure in skeletal muscle of OZR manifesting the metabolic syndrome. Further, the present study also attempted to demonstrate differences in treatment effectiveness of more recently developed statin medications as compared to traditional anti-cholesterol medications that do not fall into this category. The tested hypothesis was that chronic ingestion of anti-cholesterol therapies would better maintain microvascular structure and function in OZR, although the benefit of these effects would be due to 
improvements in vascular NO bioavailability and chronic inflammation, rather than effects on plasma cholesterol per se.

\section{MATERIALS AND METHODS}

Animals: Male lean (LZR) and OZR (Harlan) fed standard chow and drinking water (see below) ad libitum were housed at the West Virginia University Health Sciences Center and all protocols received prior IACUC approval. At 6-7 weeks of age, LZR and OZR were divided into five groups within each strain:

1. control (maintained on normal chow)

2. treatment with gemfibrozil [GEM; maintained on chow containing $50 \mathrm{mg} / \mathrm{kg} / \mathrm{d}$ gemfibrozil, a fibric acid derivative and PPAR $\alpha$ agonist (24)],

3. treatment with probucol [PRO; maintained on chow containing $100 \mathrm{mg} / \mathrm{kg} / \mathrm{d}$ probucol, an agent which increases fractional rate of LDL catabolism during cholesterol elimination. While probucol has moderate anti-oxidant properties, these appear to be a function of the LDL reducing effects of the drug rather than direct anti-oxidant effects (24)].

4. treatment with simvastatin [SIM: maintained on chow containing $20 \mathrm{mg} / \mathrm{kg} / \mathrm{d}$ simvastatin, a cholesterol lowering agent via potent inhibition of HMG Co-A reductase $(24,53)$, which also possesses anti-inflammatory properties associated with improved NO bioavailability $(12,26,28)]$.

5. treatment with atorvastatin [ATOR; maintained on chow containing $10 \mathrm{mg} / \mathrm{kg} / \mathrm{d}$ atorvastatin, a cholesterol lowering agent via potent inhibition of HMG Co-A reductase $(24,53)$, which also possesses anti-inflammatory properties associated with improved 
NO bioavailability $(4,25)]$. The primary difference between SIM and ATOR may be that SIM has a greater capacity to elevate HDL-C than ATOR $(9,24,28)$.

Rats were maintained on each of these groups for 3-4 weeks, 6-7 weeks or 10-11 weeks, at which time animals were used for experimentation (at 10, 13 and 17 weeks of age, respectively). On the day of the experiment, following an 8 hour fasting period, rats were anesthetized with injections of sodium pentobarbital (50 mg/kg, i.p.), and received tracheal intubation to facilitate maintenance of a patent airway. In all rats, a conduit artery was cannulated for determination of arterial pressure and for infusion of supplemental anesthetic and drugs, as necessary. Blood samples were drawn from the cannula for determination of glucose and insulin concentrations (Linco) as well as cholesterol and triglyceride levels (Waco). Plasma markers of inflammation were determined using commercially available ELISA systems (Luminex; Linco).

Preparation of Isolated Skeletal Muscle Resistance Arterioles: In all rats, the intramuscular continuation of the right gracilis arteriole was removed and cannulated (19). Within an individual group (above), vessels were divided into two sub-groups following an equilibration period. Group 1 examined dilator responses, where arteriolar reactivity was evaluated in response to application of acetylcholine $\left(10^{-10} \mathrm{M}-10^{-5} \mathrm{M}\right)$ and sodium nitroprusside $\left(10^{-6} \mathrm{M}\right)$ to assess reactivity to NO from both endotheliumdependent and independent agonists, respectively. Subsequently, vessels were treated with TEMPOL $\left(10^{-4} \mathrm{M}\right)$ to assess the contribution of vascular oxidant tone to agonistinduced dilation. Group 2 examined constrictor reactivity, and mechanical responses were determined following challenge with phenylephrine $\left(10^{-10} \mathrm{M}-10^{-7} \mathrm{M}\right)$. Subsequently, vessels were treated with TEMPOL or L-NAME $\left(10^{-4} \mathrm{M}\right)$ to assess the 
contribution of vascular oxidant tone and endothelium-dependent NO production to the adrenergic constriction.

In all vessels, following the completion of the above procedures, the perfusate and superfusate were replaced with $\mathrm{Ca}^{2+}$-free PSS and vessels were treated with $10^{-7} \mathrm{M}$ phenylephrine until all tone was abolished. At this time, intralumenal pressure within the vessel was altered, in $20 \mathrm{mmHg}$ increments, between $0 \mathrm{mmHg}$ and $160 \mathrm{mmHg}$ and the inner and outer diameter of arterioles was determined at each pressure. These data were used to calculate arteriolar wall mechanics which were used as indicators of structural alterations to the microvessel wall $(2,20)$.

Measurement of Vascular NO Bioavailability: From a cohort of rats within the oldest group (17 weeks of age), the abdominal aorta was removed and vascular NO production was assessed using amperometric sensors (World Precision Instruments). Briefly, aortae were isolated, sectioned longitudinally, pinned in a silastic coated dish and superfused with warmed $\left(37^{\circ} \mathrm{C}\right)$ PSS equilibrated with $95 \% \mathrm{O}_{2}$ and $5 \% \mathrm{CO}_{2}$. The NO sensor (ISO-NOPF 100) was placed in close apposition to the endothelial surface and a baseline level of current was obtained. Subsequently, increasing concentrations of methacholine $\left(10^{-10}-10^{-6} \mathrm{M}\right)$ were added to the bath and the changes in current were determined. To verify that responses represented NO release, these procedures were repeated following treatment of the aortic strip with L-NAME $\left(10^{-4} \mathrm{M}\right)$.

Histological Determination of Microvessel Density: At the conclusion of all muscle perfusion protocols, the gastrocnemius muscle from the left leg was removed, rinsed in PSS and fixed in $0.25 \%$ formalin. Muscles were embedded in paraffin and cut into $5 \mu \mathrm{m}$ cross sections. Sections were incubated with Griffonia simplicifolia I lectin (Sigma), for 
subsequent determination of microvessel density $(20,21)$.

Analyses of eNOS Expression and Activity: For determination of NOS expression, skeletal muscle arteries were homogenized and proteins within the homogenate were separated under denaturing conditions on an $8 \%$ SDS-polyacrilamide gel, after which proteins were transferred to a PVDF membrane and blocked. Subsequently, blots were incubated with mouse anti-eNOS/NOS Type III mAb (BD Transduction Laboratories), washed and incubated with appropriate horseradish peroxidase conjugated secondary antibody. GE Healthsciences ECL advance kits were used to visualize proteins. Additionally, in the oldest cohort of rats, the ascending and thoracic aorta, along with non-cannulated carotid, femoral, saphenous and iliac arteries were removed and frozen in liquid $\mathrm{N}_{2}$, for the subsequent determination of NOS activity using a commercially available kit (Cayman).

Experimental Protocols: Initially, the right gracilis muscle resistance arteriole was removed for the evaluation of vascular reactivity and passive mechanical characteristics of the vessel wall, described above. Upon completion of these procedures, the right gastrocnemius muscle was removed, cleared of non-muscular tissue, and its mass was determined. This muscle was then used for determination of microvessel density, as described above. Finally, the contralateral gastrocnemius muscle was removed for tissue banking and the aortic and arterial segments were removed for assessment of NO bioavailability and eNOS expression/activity, described above.

Data and Statistical Analyses: Mechanical responses of aortic rings following challenge with acetylcholine or phenylephrine were fit with the three-parameter logistic equation:

$$
y=\min +\left[\frac{\max -\min }{1+10^{\log E D_{50}-x}}\right]
$$


where $y$ represents the change in isometric tension, "min" and "max" represent the lower and upper bounds, respectively, of the change in arteriolar diameter with increasing agonist concentration, $x$ is the logarithm of the agonist concentration and $\log E D_{50}$ represents the logarithm of the agonist concentration $(x)$ at which the response $(y)$ is halfway between the lower and upper bounds.

Data are presented as mean \pm SEM. Statistically significant differences in measured and calculated parameters in the present study were determined using analysis of variance (ANOVA). In all cases, Student-Newman-Keuls post hoc test was used when appropriate and $p<0.05$ was taken to reflect statistical significance. For analyses of inflammatory markers between groups, we employed discriminant techniques to eliminate the univariate nature of ANOVA and issues of independent variable co-linearity which minimizes the utility of regression techniques. Discriminant analyses are based on canonical correlation to maximize differences between a priori identified experimental groups. Multiple variables are arranged into structural equations based upon their ceteris paribus ability to distinguish those group differences. These stepwise analyses result in a rank ordering of correlation coefficients in terms of their significance for the establishment of differences between groups. These produce a series of algebraic functions which explain the differences between group centroids in the " $x$ " dimension (Function 1), the

"y" dimension (Function 2). Each function explains a specific percentage of the variance between the group centroids.

\section{RESULTS}

Table 1 presents basic characteristics of animal groups within the present study. OZR were consistently heavier than LZR, and this was not impacted by the anti-cholesterol 
therapies. While OZR ultimately developed a moderate hypertension as compared to LZR, the development of the elevated blood pressure was attenuated in OZR-SIM and OZR-ATOR groups. Fasting glucose was elevated in all OZR versus age matched LZR after 10 weeks of age, although glycemic control was improved in OZR-SIM and OZRATOR groups, as the level of blood glucose was associated with reduced plasma insulin with increasing age. The hypercholesterolemia in OZR was largely abolished as a result of the four anti-cholesterol therapies, and there was a modest blunting of the hypertriglyceridemia as a result of the PRO, SIM and ATOR treatments with age. Finally, plasma levels of nitrotyrosine were elevated with age in OZR versus LZR, indicative of a chronic elevation in oxidant stress, and this difference was blunted by chronic treatment with probucol, simvastatin and atorvastatin.

The changes to skeletal muscle microvessel density with time in LZR and OZR, and the impact of chronic treatment with the anti-cholesterol agents are summarized in Figure 1. Microvessel density was not significantly impacted by the evolution of the metabolic syndrome at 7 weeks of age between LZR and OZR (Panel A), and as such, none of the pharmacological treatments impacted this relationship. However, with increasing severity of the metabolic syndrome over time, the degree of microvascular rarefaction was increased between OZR and LZR (Panels B-D). Chronic treatment with GEM and PRO had minor impacts on rarefaction in OZR, despite their effectiveness in reducing total cholesterol. In contrast, chronic treatment with either SIM or ATOR was effective at delaying/blunting the progression of microvascular rarefaction in OZR at 10, 13 and 17 weeks of age. 
At both 7 (Panel A) and 10 weeks (Panel B) of age, regardless of experimental group, both the arteriolar incremental distensibility and the circumferential stress versus strain relationship (panel inset) was not different between LZR and OZR (Figure 2). However, in both 13 week (Panel C) and 17 week (Panel D) old OZR, the slope coefficient describing this parameter was significantly elevated in OZR as compared to LZR under control conditions. While the anti-cholesterol therapies did not significantly impact this shift in the stress versus strain relationship between arterioles of LZR and OZR at 13 weeks of age, treatment with SIM or ATOR blunted this difference at 17 weeks of age, such that the slope coefficients exhibited an intermediate phenotype between that for untreated LZR and OZR.

Data describing arteriolar dilation in response to challenge with acetylcholine and the impact of acute treatment of vessels with TEMPOL on the upper bound of this relationship are summarized in Figure 3. At 7 weeks of age, the acetylcholine-induced dilator reactivity of resistance arterioles from LZR and OZR was comparable, regardless of experimental groups (Panel A) and the impact of anti-oxidant treatment on this response was negligible (Panel B). A similar situation is present at 10 weeks of age, although separation in the acetylcholine-induced dilation of resistance arterioles, while not statistically significant between LZR and OZR, is becoming apparent (Panel C). However, by 13 weeks of age (Panels $E$ and F), and even more evident at 17 weeks of age (Panels $G$ and $H$ ), acetylcholine-induced dilation was significantly reduced in OZR versus $L Z R$, and this separation was blunted by treatment with SIM and ATOR. The impact of TEMPOL treatment on this response also began to demonstrate differences, as treatment of arterioles from OZR and OZR-GEM with the anti-oxidant resulted in a significant 
improvement in the upper bound of the acetylcholine-induced response. In all cases, treatment of vessels with L-NAME abolished arteriolar responses to acetylcholine, and arteriolar dilation in response to challenge with sodium nitroprusside was not different between groups (data not shown).

Regardless of the pharmacological intervention imposed, arterial expression of eNOS between LZR and OZR did not demonstrate a consistent or significant difference between or within the two strains. Further, there was no evidence of a consistent or significant impact of age on eNOS expression between LZR and OZR (data not shown).

Figure 4 summarizes arterial eNOS activity and methacholine-induced NO production in the oldest cohort of LZR and OZR under the conditions of the present study. At 17 weeks of age, eNOS activity (Panel A) was not significantly different between LZR and OZR, and treatment with the anti-cholesterol therapies was without consistent effect. Additionally, methacholine-induced production of NO from arterial strips, assessed using amperometric sensors, was attenuated in OZR versus LZR (Panel B), although chronic ingestion of SIM or ATOR increased NO production in response to challenge with methacholine in OZR.

Data describing arteriolar constrictor responses in vessels from LZR and OZR following challenge with phenylephrine under the conditions of the present study are summarized in Figure 5. At 7 (Panel A) and 10 (Panel C) weeks of age, challenge with increasing concentrations of phenylephrine produced similar arteriolar constrictor response in OZR versus LZR under control conditions, and this relationship was not impacted by any of the anti-cholesterol therapies. Further, inhibition of eNOS enzymes with L-NAME had no impact on these responses at either 7 weeks (Panel B) or 10 weeks (Panel D) of age. 
However, by 13 weeks of age, OZR began to exhibit an increased constrictor response to phenylephrine (Panel E), and this effect was even more pronounced at 17 weeks of age (Panel G). This increased constrictor response was largely unaltered by chronic treatment with GEM or PRO at either age, although chronic treatment with SIM and ATOR blunted the augmentation to phenylephrine-induced constriction with age in OZR. Acute inhibition of eNOS with L-NAME had no effect on the differences in phenylephrine-induced constriction at 13 (Panel F) and 17 (Panel H) weeks of age between LZR and OZR under control conditions or following chronic treatment with GEM, but attenuated the impact of SIM and ATOR on moderating the increased adrenergic reactivity in OZR with age. Acute application of phentolamine $\left(10^{-5} \mathrm{M}\right)$ abolished all arteriolar responses to phenylephrine (data not shown).

Data describing the changes in plasma markers of inflammation across groups are summarized in Table 2. As compared to marker concentration in LZR, OZR demonstrated significant elevations in plasma concentrations of IL-1 $1 \beta, \mathrm{IL}-6, \mathrm{IL}-10$, TNF- $\alpha$, RANTES, MCP-1 and VEGF between 7 and 17 weeks of age. In general, GEM treatment had a minimal impact on these relationships in OZR, regardless of animal age, and PRO treatment reduced the elevation in RANTES (regulated upon activation normal T-cell expressed and secreted) at 17 weeks only. In contrast, treatment of OZR with SIM or ATOR altered levels of all markers by 13 weeks of age, except VEGF, such that their plasma concentration was significantly different from that in OZR under control conditions. Regardless of treatment, plasma concentrations of VEGF were elevated in OZR versus LZR. 
Figure 6 summarizes data from 17 week old animals from all experimental groups correlating gastrocnemius muscle microvessel density with plasma total cholesterol (Panel A) or vascular NO bioavailability (Panel B), with values from individual animals being presented. As evident from Panel A, plasma total cholesterol is a poor predictor of rarefaction and does not effectively explain the variability in muscle vascularity in the present study. In contrast, NO bioavailability, estimated from the magnitude of the upper bound from the three parameter logistic equation describing the acetylcholine concentration-response curve for individual arterioles, was a much stronger predictor of muscle vascularity, as this parameter explained almost $81 \%$ of the variability in vessel density in the present study. This relationship is evident at other ages as well (data not shown).

Table 3 presents the results of stepwise discriminant analysis based on inflammatory marker data across the experimental groups. These analyses suggest that, while most measured markers of inflammation were well correlated with microvessel density and NO bioavailability, IL-10 and MCP-1 were consistent contributors to differences not only across the experimental groups, but also over time within individual groups. In contrast, plasma RANTES levels became increasingly significant with time in terms of discriminating between groups. Alternatively, plasma TNF- $\alpha$, demonstrated an inverse relationship, become less significant with time, to the point where it dropped out of the model entirely. These data are presented graphically in Figure 7. Plasma levels of VEGF, IL-6 and IL-1 $\beta$, while well correlated with microvessel density based on univariate analyses did not contribute to the ability to discriminate between groups, and as such are not presented in Table 3. 
The relationships between these identified markers, NO bioavailability and microvessel density between 17 week old LZR and OZR is presented in Figure 8. RANTES (Panel A), IL-10 (Panel B), MCP-1 (Panel C), and TNF- $\alpha$ (Panel D) all demonstrate a strong correlation with NO bioavailability and microvessel density across the animal groups.

\section{DISCUSSION}

As a contributing component to the metabolic syndrome, OZR develop moderate hypercholesterolemia, in addition to other co-morbidities associated with this multipathology state. While previous studies have suggested that elevated plasma cholesterol can be associated with reduced capillary density $(7,42)$ and blunted angiogenic responses $(36,45)$, it is presently unclear as to the contribution of inflammatory status in terms of mediating these effects. Given this, the present study was designed to chronically treat OZR with multiple mechanistically distinct anti-cholesterol therapies throughout the period in which microvessel loss is established to better distinguish the role for plasma cholesterol in terms of its contribution to skeletal muscle microvascular rarefaction, and the role of chronic inflammation in this process.

The most immediate observation of the current study was that treatment of OZR with the anti-cholesterol agents all resulted in a comparable reduction in hypercholesterolemia at each age, despite mechanistically divergent routes of action. Probucol lowers cholesterol by increasing the fractional rate of LDL catabolism in the metabolic pathway for cholesterol elimination from the body, and may inhibit early stages of cholesterol biosynthesis (24). Gemfibrozil, a fibric acid derivative, is a PPAR $\alpha$ agonist and can reduce plasma LDL through multiple routes, including cholesteryl ester 
transfer protein activity, an increased binding affinity of LDL to its receptors, and an increased expression of hepatic LDL receptors (24). Atorvastatin and simvastatin are HMG CoA reductase inhibitors, and as such exert their anti-cholesterol actions via an inhibition of this rate limiting step in cholesterol biosynthesis. This inhibition of hepatic cholesterologenesis results in an increased expression of hepatic LDL receptors, and leads to lowered circulating cholesterol levels $(24,27)$.

Although each of these therapies was comparable in terms of efficacy in reducing plasma total cholesterol, considerable divergence was evident for their ability to ameliorate microvascular rarefaction and improve arteriolar reactivity. From a structural perspective, despite reductions in total cholesterol, treatment with gemfibrozil (and to a lesser extent probucol) had a minimal impact on blunting microvascular rarefaction with age in OZR. In contrast, chronic statin therapy lessened the severity of rarefaction, such that microvessel density assumed a level that, although reduced from LZR, was consistently improved versus untreated OZR. This observation is intriguing in that recent studies have identified a biphasic, lipid-independent, effect of chronic statin therapy on microvessel density. Specifically, it may be that low-dose statin therapy is associated with a "pro-angiogenic" environment through activation of Akt and increased NO bioavailability, whereas higher statin doses can cause angiostatic effects which are potentially mediated through a decreased protein prenylation and an inhibition of cell growth $(4,37,39,49)$. With regard to mechanics of the microvessel wall in OZR, an ameliorative effect was also identified, as the severe reduction in wall distensibility in OZR was modestly attenuated as a result of chronic ingestion of atorvastatin or simvastatin, with lesser impacts from probucol or gemfibrozil. These results support 
those determined previously, as statin therapy has been effective in reducing wall stiffness in hypercholesterolemia $(38,40)$, although additional studies have suggested that this effect may be independent of lipid profiles (44), and may better represent the pleiotropic effects of statins on endothelial function or inflammatory status.

In our previous studies, we have provided evidence suggesting one of the main causative mechanisms associated with microvascular rarefaction in OZR may be a chronic reduction in vascular NO bioavailability (16). The present results support this hypothesis, as both vascular reactivity to acetylcholine (Figure 3) and methacholineinduced NO production (Figure 4) were improved with statin therapy in OZR. Further, when data from an age cohort of animals are summarized, an index of vascular NO bioavailability (upper bound of the acetylcholine concentration-dilator response relationship) was a strong predictor of gastrocnemius muscle microvessel density (Figure 6).

The increased vascular NO bioavailability as a result of chronic atorvastatin or simvastatin treatment was also evident with regard to phenylephrine-induced constrictor reactivity. Specifically, the increased constrictor response of arterioles of OZR (versus LZR) in response to adrenergic stimulation was not impacted by chronic anti-cholesterol therapy with gemfibrozil. However, chronic treatment of OZR with atorvastatin, simvastatin or probucol blunted the upper bound of this response, although responses were still increased versus those in LZR. This may have been due to an increased vascular NO bioavailability, as acute application of L-NAME, while having minimal impact on phenylephrine-induced arteriolar constriction in OZR ( \pm gemfibrozil), increased the upper bound of this relationship in OZR treated with either of the other three agents. These data 
clearly suggest that chronic treatment with anti-cholesterol agents of the "statin" family will increase the vascular NO bioavailability (and reduce vascular oxidant stress) in OZR, and that this relationship can impact both vascular reactivity (both dilator and constrictor) and microvascular network structure.

One of the profound implications for hypercholesterolemia is the genesis of an elevated state of inflammation $(8,29,30)$, that can be associated with evolution of peripheral vasculopathy $(34,41,46)$. While inherent in the treatment of hypercholesterolemia is a reduction of plasma cholesterol, amelioration of inflammation, and improved outcomes, a key observation from the present study was that although each treatment was effective in reducing cholesterol in OZR, there was a considerable disparity in terms of these other processes. In the OZR model of the metabolic syndrome, we (13) and others $(1,33)$ have clearly demonstrated an elevated state of inflammation and that specific markers of inflammation can be strongly correlated with vascular outcomes (positively or negatively, depending on marker and outcome). As the metabolic syndrome is, by definition, composed of multiple pro-inflammatory pathologies (8), changes in multiple markers of inflammation between groups were each significant (Table 2). Further, given that indices of microvascular dysfunction were also tightly associated with animal group, as well as NO bioavailability and inflammatory state severity, significant correlations were also determined between individual markers of inflammation and specific indices of vascular dysfunction. While interesting from a conceptual standpoint, these results are largely uninformative with respect to evaluating mechanistic bases of inflammation-induced vascular dysfunction in OZR. Further, additional insight cannot effectively be gleaned from multivariate regression techniques, as the independent variables (i.e., markers of 
inflammation) demonstrate a greater co-linearity amongst themselves than with an outcome (e.g., microvessel density).

As a result, we incorporated the use of discriminant analyses (Figure 7 and Table 3), a classification technique for evaluating how multiple variables contribute to differences between experimental groups and one which is specifically designed for accurate hypothesis development. These analyses result in a rank ordering of ceteris paribus correlation coefficients in terms of significance for the establishment of differences between groups. The beneficial impact of discriminant analyses is that through a series of iterations, we are able to identify which parameters are most significant, and also which parameters are significant only because they operate through the magnitude of others. As an example, while results from ANOVA or regression suggest that IL-1 $\beta$ or IL-6 are correlated with low NO bioavailability, they suffer from considerably co-linearity with other predictive markers, limiting their utility. In contrast, discriminant analyses suggests that these markers may be less important than RANTES, MCP-1, IL-10, and TNF- $\alpha$, and may not directly contribute to differences between LZR and OZR under control conditions and following chronic anti-cholesterol therapy. These parameters identified from discriminant analyses can be used for the more targeted model development and future hypothesis testing. It is important to emphasize that these procedures do not result in an identification of the four key markers contributing to vasculopathy in the metabolic syndrome. Rather, given the limited data set employed in the present study, these analyses indicate how four markers, each of which demonstrates a strong univariate association with the vascular outcomes vary over time in terms of their ability to discriminate an outcome between 
experimental groups. With ongoing study and the inclusion of additional discriminating elements, the results of these procedures will change, although the ultimate goal is the convergence on a more stable predictive model with increasing iterations.

One of the interesting observations of the present study was while chronic treatment with the anti-cholesterol agents reduced total cholesterol in OZR to levels that were comparable to that in LZR, treatment with either of the two statin medications had the additional benefit of also reducing mean arterial pressure and improving glycemic control (i.e., insulin sensitivity) as well as providing a modest lowering of the hypertriglyceridemia in OZR. Given that the statin medications were also the most effective in terms of improving microvascular outcomes in the present study, it may be that the combination of these associated beneficial impacts of statin therapy on the other contributing pathologies of the metabolic syndrome in OZR also represented substantial mediators of the improvement to microvascular function. While an appealing possibility, the current data only allow for speculation on this hypothesis, as additional control experiments would be required to evaluate the role for these additional elements. However, this is an area of investigation that may warrant additional investment.

In summary, while development of moderate hypercholesterolemia in OZR is associated with impaired microvascular function, chronic treatment of elevated cholesterol levels does not per se result in a significant improvement to poor outcomes. Rather, only anti-cholesterol treatments that improved vascular NO bioavailability and attenuated the chronic inflammation in OZR (statins) were successful in improving microvascular and perfusion outcomes, suggesting that total plasma cholesterol itself 
may be a poor predictor of vascular outcomes in this model. Chronic inflammation, when combined with vascular NO bioavailability, may be far stronger than total cholesterol as a predictor of vascular dysfunction, and the implementation of discriminant analyses for changes to the inflammatory profile may reflect a more informative means through which future experiments may be designed. 
Reference List

1. Barbato, J.E., B.S. Zuckerbraun, M. Overhaus, K.G. Raman and E. Tzeng. Nitric oxide modulates vascular inflammation and intimal hyperplasia in insulin resistance and the metabolic syndrome. Am J Physiol Heart Circ Physiol. 289:H228-236, 2005.

2. Baumbach, G.L. and M.A. Hajdu. Mechanics and composition of cerebral arterioles in renal and spontaneously hypertensive rats. Hypertension. 21:816-826, 1993.

3. Beckman, J.A. and M.A. Creager. The nonlipid effects of statins on endothelial function. Trends Cardiovasc Med. 16:156-162, 2006.

4. Boodhwani, M., Y. Nakai, P. Voisine, J. Feng, J. Li, S. Mieno, B. Ramlawi, C. Bianchi, R. Laham and F.W. Sellke. High-dose atorvastatin improves hypercholesterolemic coronary endothelial dysfunction without improving the angiogenic response. Circulation. 114:1402-1408, 2006.

5. Bray, G.A. The Zucker-fatty rat: a review. Fed Proc. 36:148-153, 1977.

6. Brzezinska, A.K., D. Gebremedhin, W.M. Chilian, B. Kalyanaraman and S.J. Elliott. Peroxynitrite reversibly inhibits $\mathrm{Ca}(2+)$-activated $\mathrm{K}(+)$ channels in rat cerebral artery smooth muscle cells. Am J Physiol Heart Circ Physiol. 278:H1883H1890, 2000.

7. Bucay, M., J. Nguy, R. Barrios, C.H. Chen and P.D. Henry. Impaired adaptive vascular growth in hypercholesterolemic rabbit. Atherosclerosis. 139:243-251, 1998. 
8. Dandona, P., A. Aljada, A. Chaudhuri, P. Mohanty and R. Garg. Metabolic syndrome: a comprehensive perspective based on interactions between obesity, diabetes, and inflammation. Circulation. 111:1448-1454, 2005.

9. Davidson, M.H., L. Ose, J. Frohlich, R.S. Scott, C.A. Dujovne, I.D. Escobar, M.C. Bertolami, F. Cihon, D.L. Maccubbin, M. Mercuri. Differential effects of simvastatin and atorvastatin on high-density lipoprotein cholesterol and apolipoprotein A-I are consistent across hypercholesterolemic patient subgroups. Clin Cardiol. 26:509-514, 2003.

10. Duarte, J., A. Martinez, A. Bermejo, B. Vera, M.J. Gamez, P. Cabo and A. Zarzuelo. Cardiovascular effects of captopril and enalapril in obese Zucker rats. Eur J Pharmacol. 365:225-232, 1999.

11. Endres, M. Statins: potential new indications in inflammatory conditions. Atheroscler Suppl. 7:31-35, 2006.

12. Field, K.M. Effect of 3-hydroxy-3-methylglutaryl coenzyme A reductase inhibitors on high-sensitivity C-reactive protein levels. Pharmacotherapy. 25:1365-1377, 2005.

13. Frisbee, J.C., J. Balch Samora, J. Peterson and R. Bryner. Exercise training blunts microvascular rarefaction in the metabolic syndrome. Am J Physiol Heart Circ Physiol. 291:H2483-H2492, 2006.

14. Frisbee, J.C. Impaired hemorrhage tolerance in the obese Zucker rat model of metabolic syndrome. J Appl Physiol. 100:465-473, 2006.

15. Frisbee JC. Vascular adrenergic tone and structural narrowing constrain reactive hyperemia in skeletal muscle of obese Zucker rats. Am J Physiol Heart Circ Physiol. 290:H2066-H2074, 2006. 
16. Frisbee, J.C. Reduced nitric oxide bioavailability contributes to skeletal muscle microvessel rarefaction in the metabolic syndrome. Am J Physiol Regul Integr Comp Physiol. 289:R307-R316, 2005.

17. Frisbee, J.C. Hypertension-independent microvascular rarefaction in the obese Zucker rat model of the metabolic syndrome. Microcirculation. 12:383-392, 2005.

18. Frisbee, J.C. Enhanced arteriolar alpha-adrenergic constriction impairs dilator responses and skeletal muscle perfusion in obese Zucker rats. J Appl Physiol. 97:764-772, 2004.

19. Frisbee, J.C. Impaired skeletal muscle perfusion in obese Zucker rats. Am J Physiol Regul Integr Comp Physiol. 285:R1124-R1134, 2003.

20.Frisbee, J.C. Remodeling of the skeletal muscle microcirculation increases resistance to perfusion in obese Zucker rats. Am J Physiol Heart Circ Physiol. 285:H104-H111, 2003.

21. Ginsberg, H.N. Efficacy and mechanisms of action of statins in the treatment of diabetic dyslipidemia. J Clin Endocrinol Metab. 91:383-392, 2006.

22.Greene, A.S., J.H. Lombard, A.W. Cowley Jr and F.M. Hansen-Smith. Microvessel changes in hypertension measured by Griffonia simplicifolia I lectin. Hypertension. 15:779-783, 1990.

23. Guerre-Millo, M. Regulation of ob gene and overexpression in obesity. Biomed Pharmacother. 51:318-323, 1997.

24. Hardman, J.G., L.E. Limbird and A.G. Gilman (Eds.). "Goodman and Gilman's: The Pharmacological Basis of Therapeutics" $10^{\text {th }}$ edition, McGraw-Hill, Inc., New York, NY. 2001, pp. 971-1002. 
25. Hognestad, A., P. Aukrust, R. Wergeland, O. Stokke, L. Gullestad, A.G. Semb, T. Holm, A.K. Andreassen and J.K. Kjekshus. Effects of conventional and aggressive statin treatment on markers of endothelial function and inflammation. Clin Cardiol. 27:199-203, 2004.

26. John, S., C. Delles, J. Jacobi, M.P. Schlaich, M. Schneider, G. Schmitz, R.E. Schmieder. Rapid improvement of nitric oxide bioavailability after lipid-lowering therapy with cerivastatin within two weeks. J Am Coll Cardiol. 37:1351-1358, 2001.

27.Knopp, R.H. and P. Paramsothy. Treatment of hypercholesterolemia in patients with metabolic syndrome: how do different statins compare? Nat Clin Pract Endocrinol Metab. 2:136-137, 2006.

28. Laufs, U., V. La Fata, J. Plutzky and J.K. Liao. Upregulation of endothelial nitric oxide synthase by HMG CoA reductase inhibitors. Circulation. 97:1129-1135, 1998.

29. Libby, P. Inflammation in atherosclerosis. Nature. 420:868-874, 2002.

30.Lyon, C.J., R.E. Law and W.A. Hsueh. Minireview: adiposity, inflammation, and atherogenesis. Endocrinology. 144:2195-2200, 2003.

31. Martinez-Gonzalez, J., J. Alfon, M. Berrozpe and L. Badimon. HMG-CoA reductase inhibitors reduce vascular monocyte chemotactic protein-1 expression in early lesions from hypercholesterolemic swine independently of their effect on plasma cholesterol levels. Atherosclerosis. 159:27-33, 2001.

32. Mason, R.P., M.F. Walter, C.A Day and R.F. Jacob. Intermolecular differences of 3-hydroxy-3-methylglutaryl coenzyme a reductase inhibitors contribute to distinct pharmacologic and pleiotropic actions. Am J Cardiol. 96:11F-23F, 2005. 
33.Picchi, A., X. Gao, S. Belmadani, B.J. Potter, M. Focardi, W.M. Chilian and C. Zhang. Tumor necrosis factor-alpha induces endothelial dysfunction in the prediabetic metabolic syndrome. Circ Res. 99:69-77, 2006.

34.Pirro, M., G. Schillaci, G. Savarese, F. Gemelli, G. Vaudo, D. Siepi, F. Bagaglia and E. Mannarino. Low-grade systemic inflammation impairs arterial stiffness in newly diagnosed hypercholesterolaemia. Eur J Clin Invest. 34:335-341, 2004.

35.Ray, K.K. and C.P. Cannon. The potential relevance of the multiple lipidindependent (pleiotropic) effects of statins in the management of acute coronary syndromes. J Am Coll Cardiol. 46:1425-1433, 2005.

36. Sasaki, K., J. Duan, T. Murohara, H. Ikeda, S. Shintani, T. Shimada, T. Akita, K. Egami and T. Imaizumi. Rescue of hypercholesterolemia-related impairment of angiogenesis by oral folate supplementation. J Am Coll Cardiol. 42:364-372, 2003.

37. Sata, M., H. Nishimatsu, J. Osuga, K. Tanaka, N. Ishizaka, S. Ishibashi, Y. Hirata and R. Nagai. Statins augment collateral growth in response to ischemia but they do not promote cancer and atherosclerosis. Hypertension. 43:1214-1220, 2004.

38. Shige, H., A. Dart and P. Nestel. Simvastatin improves arterial compliance in the lower limb but not in the aorta. Atherosclerosis. 155:245-250, 2001.

39. Skaletz-Rorowski, A. and K. Walsh. Statin therapy and angiogenesis. Curr Opin Lipidol. 14:599-603, 2003.

40.Smilde, T.J., F.W. van den Berkmortel, H. Wollersheim, H. van Langen, J.J. Kastelein and A.F. Stalenhoef. The effect of cholesterol lowering on carotid and 
femoral artery wall stiffness and thickness in patients with familial hypercholesterolaemia. Eur J Clin Invest. 30:473-480, 2000.

41. Stokes, K.Y. Microvascular responses to hypercholesterolemia: the interactions between innate and adaptive immune responses. Antioxid Redox Signal. 8:11411151, 2006.

42. Theilmeier G, Verhamme P, Dymarkowski S, Beck H, Bernar H, Lox M, Janssens S, Herregods MC, Verbeken E, Collen D, Plate K, Flameng W, Holvoet P. Hypercholesterolemia in minipigs impairs left ventricular response to stress: association with decreased coronary flow reserve and reduced capillary density. Circulation. 106:1140-1146, 2002.

43. Tofovic, S.P. and E.K. Jackson. Rat models of the metabolic syndrome. Methods Mol Med. 86:29-46, 2003.

44.Ubels, F.L., J.H. Muntinga, J.J. van Doormaal, W.D. Reitsma and A.J. Smit. Effects of initial and long-term lipid-lowering therapy on vascular wall characteristics. Atherosclerosis. 154:155-161, 2001.

45. Van Belle, E., A. Rivard, D. Chen, M. Silver, S. Bunting, N. Ferrara, J.F. Symes, C. Bauters and J.M. Isner. Hypercholesterolemia attenuates angiogenesis but does not preclude augmentation by angiogenic cytokines. Circulation. 96:2667-2674, 1997.

46.van Haelst, P.L., J.J. van Doormaal, F.W. Asselbergs, A.M. van Roon, N.J. Veeger, M.M. Henneman, A.J. Smit, J.W. Tervaert, J.F. May and R.O. Gans. Correlates of endothelial function and their relationship with inflammation in patients with familial hypercholesterolaemia. Clin Sci (Lond). 104:627-632, 2003. 
47.Vaziri, N.D., Z.G. Xu, A. Shahkarami, K.T. Huang, B. Rodriguez-Iturbe and R. Natarajan. Role of AT-1 receptor in regulation of vascular MCP-1, IL-6, PAI-1, MAP kinase, and matrix expressions in obesity. Kidney Int. 68:2787-2793, 2005.

48. Walker, A.B., P.D. Chattington, R.E. Buckingham and G. Williams. The thiazolidinedione rosiglitazone (BRL-49653) lowers blood pressure and protects against impairment of endothelial function in Zucker fatty rats. Diabetes. 48:14481453, 1999.

49. Weis, M., C. Heeschen, A.J. Glassford and J.P. Cooke. Statins have biphasic effects on angiogenesis. Circulation. 105:739-745, 2002.

50.Xiang, L., J.S. Naik, S.R. Abram and R.L. Hester. Chronic hyperglycemia impairs functional vasodilation via increasing thromboxane receptor-mediated vasoconstriction. Am J Physiol Heart Circ Physiol. [Epub ahead of print], 2006.

51.Xiang, L., J.S. Naik, B.L. Hodnett and R.L. Hester. Altered arachidonic acid metabolism impairs functional vasodilation in metabolic syndrome. Am J Physiol Regul Integr Comp Physiol. 290:R134-R138, 2006.

52.Xiang, L., J. Naik and R.L. Hester. Exercise-induced increase in skeletal muscle vasodilatory responses in obese Zucker rats. Am J Physiol Regul Integr Comp Physiol. 288:R987-R991, 2005.

53.Zhou, Z., E. Rahme and L. Pilote. Are statins created equal? Evidence from randomized trials of pravastatin, simvastatin, and atorvastatin for cardiovascular disease prevention. Am Heart J. 151:273-281, 2006. 
Table 1. Characteristics of animal groups within the present study. ${ }^{*} p<0.05 \mathrm{vs.} \mathrm{LZR;} \dagger$ p $<0.05$ vs. OZR.

\begin{tabular}{|c|c|c|c|c|c|c|c|}
\hline & Age & LZR & OZR & OZR-GEM & OZR-PRO & OZR-SIM & OZR-ATOR \\
\hline \multirow[t]{4}{*}{ Mass (g) } & $7 w$ & $155 \pm 5$ & $241 \pm 6^{*}$ & $254 \pm 8^{*}$ & $250 \pm 9^{*}$ & $247 \pm 8^{*}$ & $245 \pm 5^{*}$ \\
\hline & $10 w$ & $238 \pm 6$ & $401 \pm 8^{*}$ & $398 \pm 7^{*}$ & $400 \pm 9^{*}$ & $405 \pm 8^{*}$ & $408 \pm 7^{*}$ \\
\hline & $13 w$ & $304 \pm 7$ & $513 \pm 10^{*}$ & $520 \pm 10^{*}$ & $508 \pm 12^{*}$ & $506 \pm 9^{*}$ & $501 \pm 8^{*}$ \\
\hline & $17 w$ & $369 \pm 5$ & $628 \pm 12^{*}$ & $630 \pm 12^{*}$ & $625 \pm 13^{*}$ & $622 \pm 12^{*}$ & $618 \pm 9^{*}$ \\
\hline \multirow[t]{4}{*}{ MAP (mmHg) } & $7 w$ & $98 \pm 4$ & $95 \pm 5$ & $97 \pm 5$ & $98 \pm 6$ & $93 \pm 5$ & $99 \pm 4$ \\
\hline & $10 w$ & $99 \pm 5$ & $99 \pm 5$ & $102 \pm 6$ & $100 \pm 5$ & $99 \pm 4$ & $100 \pm 5$ \\
\hline & $13 w$ & $102 \pm 5$ & $112 \pm 6$ & $115 \pm 6$ & $112 \pm 8$ & $102 \pm 6$ & $104 \pm 6$ \\
\hline & $17 w$ & $102 \pm 4$ & $129 \pm 5^{*}$ & $127 \pm 5^{*}$ & $123 \pm 4^{*}$ & $118 \pm 4^{*}$ & $113 \pm 5^{*} \dagger$ \\
\hline \multirow[t]{4}{*}{ Glucose (mg/dl) } & $7 w$ & $93 \pm 7$ & $98 \pm 4$ & $104 \pm 7$ & $102 \pm 8$ & $96 \pm 8$ & $93 \pm 6$ \\
\hline & $10 w$ & $96 \pm 6$ & $103 \pm 7$ & $105 \pm 8$ & $104 \pm 9$ & $101 \pm 9$ & $105 \pm 8$ \\
\hline & $13 w$ & $99 \pm 7$ & $123 \pm 8^{*}$ & $128 \pm 8^{*}$ & $128 \pm 8^{*}$ & $128 \pm 6^{*}$ & $121 \pm 8^{*}$ \\
\hline & $17 w$ & $106 \pm 9$ & $168 \pm 10^{*}$ & $170 \pm 8^{*}$ & $169 \pm 11^{*}$ & $150 \pm 7^{*}$ & $155 \pm 8^{*}$ \\
\hline \multirow[t]{4}{*}{ Insulin (ng/ml) } & $7 w$ & $1.0 \pm 0.3$ & $3.2 \pm 0.5^{*}$ & $4.1 \pm 0.6^{*}$ & $3.6 \pm 0.7^{*}$ & $2.9 \pm 0.5^{\star}$ & $3.0 \pm 0.6^{*}$ \\
\hline & $10 w$ & $1.1 \pm 0.2$ & $4.9 \pm 0.7^{*}$ & $5.4 \pm 0.6^{*}$ & $6.0 \pm 0.6^{*}$ & $4.8 \pm 0.6^{*}$ & $4.3 \pm 0.5^{*}$ \\
\hline & $13 w$ & $1.2 \pm 0.3$ & $7.9 \pm 0.6^{*}$ & $7.5 \pm 0.7^{*}$ & $7.8 \pm 0.8^{*}$ & $6.0 \pm 0.6^{*}$ & $5.7 \pm 0.6^{*}$ \\
\hline & $17 w$ & $1.2 \pm 0.2$ & $9.0 \pm 0.8^{*}$ & $8.2 \pm 0.6^{*}$ & $9.1 \pm 0.9^{*}$ & $6.4 \pm 0.8^{*} \dagger$ & $6.5 \pm 0.6^{*} \dagger$ \\
\hline \multirow[t]{4}{*}{ Chol (mg/dl) } & $7 w$ & $80 \pm 7$ & $98 \pm 8$ & $95 \pm 10$ & $95 \pm 9$ & $91 \pm 9$ & $94 \pm 6$ \\
\hline & $10 w$ & $82 \pm 6$ & $106 \pm 7^{*}$ & $84 \pm 8 †$ & $83 \pm 9 \dagger$ & $83 \pm 8 \dagger$ & $84 \pm 7 \dagger$ \\
\hline & $13 w$ & $77 \pm 8$ & $128 \pm 10^{*}$ & $87 \pm 9 \dagger$ & $88 \pm 9+$ & $85 \pm 9 \dagger$ & $85 \pm 9 \dagger$ \\
\hline & $17 w$ & $71 \pm 10$ & $142 \pm 12^{*}$ & $86 \pm 9 \dagger$ & $84 \pm 11 \dagger$ & $82 \pm 11 \dagger$ & $81 \pm 12 \dagger$ \\
\hline \multirow[t]{4}{*}{ TG (mg/dl) } & $7 w$ & $101 \pm 8$ & $175 \pm 9^{*}$ & $178 \pm 12^{*}$ & $176 \pm 10^{*}$ & $180 \pm 11^{*}$ & $176 \pm 11^{*}$ \\
\hline & $10 w$ & $106 \pm 7$ & $247 \pm 11^{*}$ & $249 \pm 14^{*}$ & $255 \pm 14^{*}$ & $252 \pm 12^{*}$ & $258 \pm 14^{*}$ \\
\hline & $13 w$ & $124 \pm 10$ & $341 \pm 12^{*}$ & $338 \pm 11^{*}$ & $338 \pm 13^{*}$ & $290 \pm 14^{*} \dagger$ & $302 \pm 13^{*} \dagger$ \\
\hline & $17 w$ & $140 \pm 12$ & $360 \pm 15^{\star}$ & $342 \pm 20^{*}$ & $330 \pm 14^{*} \dagger$ & $320 \pm 15^{*} \dagger$ & $316 \pm 16^{*} \dagger$ \\
\hline \multirow[t]{4}{*}{ N-tyrosine (ng/ml) } & $7 w$ & $9 \pm 2$ & $16 \pm 4$ & $17 \pm 4$ & $12 \pm 4$ & $13 \pm 4$ & $14 \pm 3$ \\
\hline & $10 w$ & $11 \pm 3$ & $25 \pm 4^{*}$ & $26 \pm 4^{*}$ & $20 \pm 3^{*}$ & $16 \pm 4$ & $15 \pm 5$ \\
\hline & $13 w$ & $12 \pm 3$ & $49 \pm 7^{*}$ & $45 \pm 7^{*}$ & $30 \pm 6^{*} \dagger$ & $24 \pm 5^{*}$ & $25 \pm 4^{*} \dagger$ \\
\hline & $17 w$ & $12 \pm 3$ & $61 \pm 6^{*}$ & $53 \pm 5^{*}$ & $40 \pm 5^{*} \dagger$ & $29 \pm 5^{*} \dagger$ & $27 \pm 5^{*} \dagger$ \\
\hline
\end{tabular}


Table 2. Plasma markers of inflammation $(\mathrm{pg} / \mathrm{ml})$ in the present study. ${ }^{*} p<0.05 \mathrm{vs}$. LZR; $\dagger p<0.05$ vs. OZR.

\begin{tabular}{|c|c|c|c|c|c|c|c|}
\hline & Age & LZR & OZR & OZR-GEM & OZR-PRO & OZR-SIM & OZR-ATOR \\
\hline \multirow[t]{4}{*}{ IL-1 $\beta$} & $7 w$ & $9.2 \pm 0.7$ & $9.4 \pm 0.6$ & $9.1 \pm 0.8$ & $8.5 \pm 1.0$ & $8.5 \pm 0.8$ & $8.4 \pm 0.5$ \\
\hline & $10 w$ & $9.4 \pm 0.6$ & $13.4 \pm 1.8^{*}$ & $12.2 \pm 1.6^{*}$ & $12.6 \pm 1.8^{*}$ & $11.0 \pm 1.4^{*}$ & $10.8 \pm 1.1^{*}$ \\
\hline & $13 w$ & $9.8 \pm 0.7$ & $18.5 \pm 2.0^{*}$ & $18.3 \pm 1.9^{*}$ & $16.8 \pm 1.7^{*}$ & $12.0 \pm 1.9^{*} \dagger$ & $12.2 \pm 1.8^{*} \dagger$ \\
\hline & $17 w$ & $10.2 \pm 0.8$ & $23.2 \pm 2.5^{\star}$ & $19.8 \pm 2.2^{*}$ & $18.5 \pm 2.0^{*}$ & $14.4 \pm 1.8 \dagger$ & $15.0 \pm 2.0 \dagger$ \\
\hline \multirow[t]{4}{*}{ IL-6 } & $7 w$ & $26.2 \pm 4.8$ & $35.5 \pm 5.1$ & $33.8 \pm 4.6$ & $29.8 \pm 5.3$ & $28.0 \pm 4.5$ & $31.1 \pm 4.4$ \\
\hline & $10 w$ & $30.8 \pm 5.4$ & $40.5 \pm 5.4$ & $36.2 \pm 6.0$ & $34.3 \pm 5.5$ & $29.9 \pm 4.3$ & $35.0 \pm 5.1$ \\
\hline & $13 w$ & $36.8 \pm 5.6$ & $68.6 \pm 6.1^{*}$ & $65.2 \pm 5.6^{*}$ & $64.6 \pm 5.8^{*}$ & $38.4 \pm 6.1 †$ & $40.6 \pm 4.6 \dagger$ \\
\hline & $17 w$ & $38.6 \pm 4.6$ & $79.8 \pm 5.7^{*}$ & $77.2 \pm 6.6^{*}$ & $71.0 \pm 6.4^{*}$ & $52.4 \pm 5.8 \dagger$ & $53.9 \pm 6.1 \dagger$ \\
\hline \multirow[t]{4}{*}{ IL-10 } & $7 w$ & $14.6 \pm 2.6$ & $24.8 \pm 4.0$ & $22.0 \pm 4.1$ & $16.5 \pm 3.8$ & $15.2 \pm 3.8$ & $14.6 \pm 3.1$ \\
\hline & $10 w$ & $15.2 \pm 3.6$ & $80.3 \pm 6.7^{*}$ & $75.3 \pm 8.0^{*}$ & $64.4 \pm 9.0^{*}$ & $51.0 \pm 6.8^{*} \dagger$ & $49.5 \pm 5.8 \dagger$ \\
\hline & $13 w$ & $16.4 \pm 3.7$ & $116.4 \pm 10.5^{*}$ & $108.8 \pm 10.4^{*}$ & $104.3 \pm 8.9^{*}$ & $68.5 \pm 7.6^{*} \dagger$ & $62.4 \pm 6.8^{*} \dagger$ \\
\hline & $17 w$ & $18.4 \pm 3.0$ & $124 \pm 11.5^{*}$ & $117.4 \pm 10.4^{*}$ & $114.4 \pm 12.0^{*}$ & $74.4 \pm 9.4^{*} \dagger$ & $68.8 \pm 11.2^{*} \dagger$ \\
\hline \multirow[t]{4}{*}{ TNF- $\alpha$} & $7 w$ & $2.0 \pm 0.4$ & $5.0 \pm 1.0^{*}$ & $5.1 \pm 0.9^{*}$ & $4.6 \pm 0.7^{*}$ & $3.9 \pm 0.7^{*}$ & $3.5 \pm 0.6^{*}$ \\
\hline & $10 w$ & $2.5 \pm 0.4$ & $8.9 \pm 1.0^{*}$ & $8.4 \pm 1.1^{*}$ & $7.2 \pm 1.2^{*}$ & $5.8 \pm 0.8^{*} \dagger$ & $6.3 \pm 1.1^{*} \dagger$ \\
\hline & $13 w$ & $3.2 \pm 0.3$ & $10.3 \pm 1.6^{*}$ & $9.8 \pm 0.9^{*}$ & $8.6 \pm 1.8^{*}$ & $5.0 \pm 1.2 \dagger$ & $5.7 \pm 1.6 \dagger$ \\
\hline & $17 w$ & $3.4 \pm 0.4$ & $10.4 \pm 1.4^{*}$ & $10.8 \pm 1.2^{*}$ & $8.8 \pm 1.5^{*}$ & $6.2 \pm 1.4 \dagger$ & $6.8 \pm 1.6 \dagger$ \\
\hline \multirow[t]{4}{*}{ RANTES } & $7 w$ & $28.0 \pm 4.7$ & $34.2 \pm 4.8$ & $35.0 \pm 5.1$ & $33.4 \pm 4.9$ & $31.3 \pm 5.5$ & $29.6 \pm 4.6$ \\
\hline & $10 w$ & $32.2 \pm 4.6$ & $66.6 \pm 7.9^{*}$ & $60.4 \pm 8.2^{*}$ & $53.4 \pm 5.3^{*}$ & $43.4 \pm 5.8 \dagger$ & $44.2 \pm 4.7 \dagger$ \\
\hline & $13 w$ & $36.4 \pm 4.8$ & $120.2 \pm 10.3^{*}$ & $117.4 \pm 9.9^{*}$ & $108.8 \pm 10.9^{*}$ & $85.4 \pm 9.9^{*} \dagger$ & $80.8 \pm 7.9^{*} \dagger$ \\
\hline & $17 w$ & $40.1 \pm 5.3$ & $187.2 \pm 14.1^{*}$ & $176.8 \pm 15.8^{*}$ & $138.4 \pm 14.8^{*} \dagger$ & $97.8 \pm 14.0^{*} \dagger$ & $102.8 \pm 13.6^{*} \dagger$ \\
\hline \multirow[t]{4}{*}{ MCP-1 } & $7 w$ & $36.6 \pm 5.8$ & $45.0 \pm 5.9$ & $43.8 \pm 5.2$ & $45.6 \pm 4.9$ & $40.4 \pm 5.1$ & $41.2 \pm 6.0$ \\
\hline & $10 w$ & $36.8 \pm 4.7$ & $57.7 \pm 10.5$ & $54.9 \pm 12.0$ & $51.5 \pm 6.4$ & $43.6 \pm 5.2^{*} \dagger$ & $42.5 \pm 5.0^{*}$ \\
\hline & $13 w$ & $42.1 \pm 4.6$ & $98.4 \pm 10.4^{*}$ & $95.6 \pm 10.6^{*}$ & $81.0 \pm 8.9^{*}$ & $66.4 \pm 9.4^{*} \dagger$ & $62.2 \pm 6.3^{*} \dagger$ \\
\hline & $17 w$ & $42.2 \pm 5.8$ & $120.2 \pm 10.8^{*}$ & $118.4 \pm 12.0^{*}$ & $116.6 \pm 14.2^{*}$ & $80.4 \pm 12.1^{*} \dagger$ & $77.8 \pm 11.4^{*} \dagger$ \\
\hline \multirow[t]{4}{*}{ VEGF } & $7 w$ & $35.6 \pm 5.0$ & $39.6 \pm 6.1$ & $37.0 \pm 4.6$ & $40.2 \pm 4.5$ & $38.3 \pm 4.3$ & $39.6 \pm 5.3$ \\
\hline & $10 w$ & $41.1 \pm 5.3$ & $55.5 \pm 4.5^{\star}$ & $56.2 \pm 4.8^{*}$ & $50.2 \pm 5.3^{*}$ & $53.4 \pm 4.9^{*}$ & $55.5 \pm 4.5^{*}$ \\
\hline & $13 w$ & $39.2 \pm 5.3$ & $62.9 \pm 6.7^{*}$ & $60.6 \pm 5.7^{*}$ & $58.6 \pm 6.1^{*}$ & $54.6 \pm 5.1^{*}$ & $56.9 \pm 6.4^{*}$ \\
\hline & $17 w$ & $38.9 \pm 4.1$ & $68.7 \pm 5.4^{*}$ & $66.2 \pm 6.1^{*}$ & $61.8 \pm 5.8^{*}$ & $57.4 \pm 4.5^{\star}$ & $58.8 \pm 4.8^{*}$ \\
\hline
\end{tabular}


Table 3. Structure matrix of results from discriminant analyses for markers of inflammation between animal groups in the present study. * represents the largest absolute correlation between each variable and any discriminant function.

\begin{tabular}{ccccccccc}
\hline Age & \multicolumn{2}{c}{$\mathbf{7}$ Weeks } & \multicolumn{2}{c}{ 10 Weeks } & \multicolumn{2}{c}{13 Weeks } & \multicolumn{2}{c}{17 Weeks } \\
\hline Functions & $\mathbf{1}$ & $\mathbf{2}$ & $\mathbf{1}$ & $\mathbf{2}$ & $\mathbf{1}$ & $\mathbf{2}$ & $\mathbf{1}$ & $\mathbf{2}$ \\
\% of Variance & 81.7 & 12.0 & 96.2 & 2.6 & 97.3 & 3.0 & 98.3 & 1.5 \\
Canonical Correlation & 0.907 & 0.636 & 0.975 & 0.581 & 0.988 & 0.746 & 0.964 & 0.405 \\
RANTES & --- & --- & 0.364 & $0.683^{*}$ & 0.504 & 0.289 & $0.647^{*}$ & $-0.760^{*}$ \\
IL-10 & 0.390 & $0.700^{*}$ & $0.531^{*}$ & -0.182 & $0.576^{*}$ & -0.389 & 0.521 & 0.593 \\
MCP-1 & 0.368 & -0.049 & 0.384 & 0.428 & 0.465 & $0.568^{*}$ & 0.501 & 0.452 \\
TNF- $\alpha$ & $0.553^{*}$ & -0.568 & 0.401 & -0.173 & 0.244 & -0.300 & --- & --- \\
\hline
\end{tabular}




\section{FIGURE LEGENDS}

Figure 1. Data (mean $\pm S E M)$ microvessel density within skeletal muscle of LZR and OZR at 7 weeks (Panel A), 10 weeks (Panel B), 13 weeks (Panel C) and 17 weeks (Panel D) of age. Microvessel density data are presented under control conditions and following chronic treatment of OZR with the anti-cholesterol therapies: gemfibrozil, probucol, simvastatin or atorvastatin. Microvessel density was determined using fluorescence microscopy following labeling of microvessel with Griffonia simplicifolia I lectin (please see text for details). ${ }^{*} p<0.05$ vs. LZR; $\dagger p<0.05$ vs. OZR.

Figure 2. Data (mean $\pm S E M)$ describing incremental distensibility and the slope $(\beta)$ coefficients from circumferential stress versus strain relationships (inset panels) of skeletal muscle arterioles of LZR and OZR at 7 weeks (Panel A), 10 weeks (Panel B), 13 weeks (Panel C) and 17 weeks (Panel D) of age. Arteriolar wall mechanics data are presented control conditions and following chronic treatment of OZR with the anti-cholesterol therapies: gemfibrozil, probucol, simvastatin or atorvastatin. ${ }^{*} p<0.05$ vs. LZR; $\uparrow p<0.05$ vs. OZR.

Figure 3. Data (mean $\pm S E M)$ describing skeletal muscle arteriolar dilation in response to increasing concentrations of acetylcholine of LZR and OZR under control conditions and following chronic treatment of OZR with gemfibrozil, probucol, simvastatin or atorvastatin. Data area presented as paired panels, with the left panels summarizing the concentrationresponse relationship, and the right panel presenting the contribution of oxidant stress in terms of impacting acetylcholine-induced dilation where the change in the upper bound of 
this relationship is shown following treatment of the arteriole with TEMPOL. Data are presented for animals at 7 weeks (Panels A/B), 10 weeks (Panels C/D), 13 weeks (Panels $E / F)$ and 17 weeks (Panels G/H) of age. ${ }^{*} p<0.05$ vs. LZR; $\uparrow p<0.05$ vs. OZR.

Figure 4. Vascular eNOS activity (Panel A; presented as the $\%$ arginine conversion), and methacholine-induced NO bioavailability (Panel B; where data present the slope of the relationship between vascular NO production and methacholine concentration, $\mathrm{nM} / \log \mathrm{M}$ methacholine) in LZR and OZR at 17 weeks of age. Data (presented as mean \pm SEM) are summarized for LZR and OZR under control conditions and following chronic treatment of OZR with gemfibrozil, probucol, simvastatin or atorvastatin. $\quad{ }^{*} p<0.05$ vs. LZR; $† p<0.05$ vs. OZR.

Figure 5. Data (mean \pm SEM) describing skeletal muscle arteriolar constriction in response to increasing concentrations of phenylephrine in LZR and OZR under control conditions and following chronic treatment of OZR with gemfibrozil, probucol, simvastatin or atorvastatin. Data area presented as paired panels, with the left panels summarizing the concentration-response relationship, and the right panel presenting the contribution of vascular nitric oxide production in terms of impacting phenylephrine-induced constriction where the change in the upper bound of this relationship is shown following treatment of the arteriole with L-NAME. Data are presented for animals at 7 weeks (Panels A/B), 10 weeks (Panels C/D), 13 weeks (Panels E/F) and 17 weeks (Panels $G / H)$ of age. ${ }^{*} p<0.05$ vs. LZR; † $p<0.05$ vs. OZR. 
Figure 6. The relation between plasma total cholesterol level (Panel A), or a proxy variable for NO bioavailability (upper bound of the acetylcholine dose-response relationship; Panel B), and microvessel density from the different animals in the present study. Each animal used in the study, across the experimental groups is presented in this figure. The inset text presents the linear regression equation that best fits these data and the degree to which that equation explains the variability in the data.

Figure 7. Summary plot for the results of the discriminant analyses in the present study. These results provide the functions 1 and 2 (presented in Table 3) which contribute the majority $(>90 \%)$ of the differences between the experimental groups at each age. Specifically, RANTES, IL-10, MCP-1 and TNF- $\alpha$ play the greatest role in terms of establishing differences between LZR (light blue), OZR (green), and OZR under the four treatment conditions of the present study; gemfibrozil (grey), probucol (purple), simvastatin (orange) and atorvastatin (red). The centroids for each group are presented in the dark blue squares.

Figure 8. Relationships between the four most significant markers of inflammation (identified using discriminant analyses; please see text for details), vascular NO bioavailability, and gastrocnemius muscle microvessel density for animals in the present study. Data are presented for RANTES (Panel A), IL-10 (Panel B), MCP-1 (Panel C), and TNF- $\alpha$ (Panel D), and the same color coding is used as in Figure 4; LZR (light blue), OZR (green), and OZR+GEM (grey), OZR+PRO (purple), OZR+SIM (orange) and OZR+ATOR (red). 


\section{FIGURES}

\section{Figure 1.}
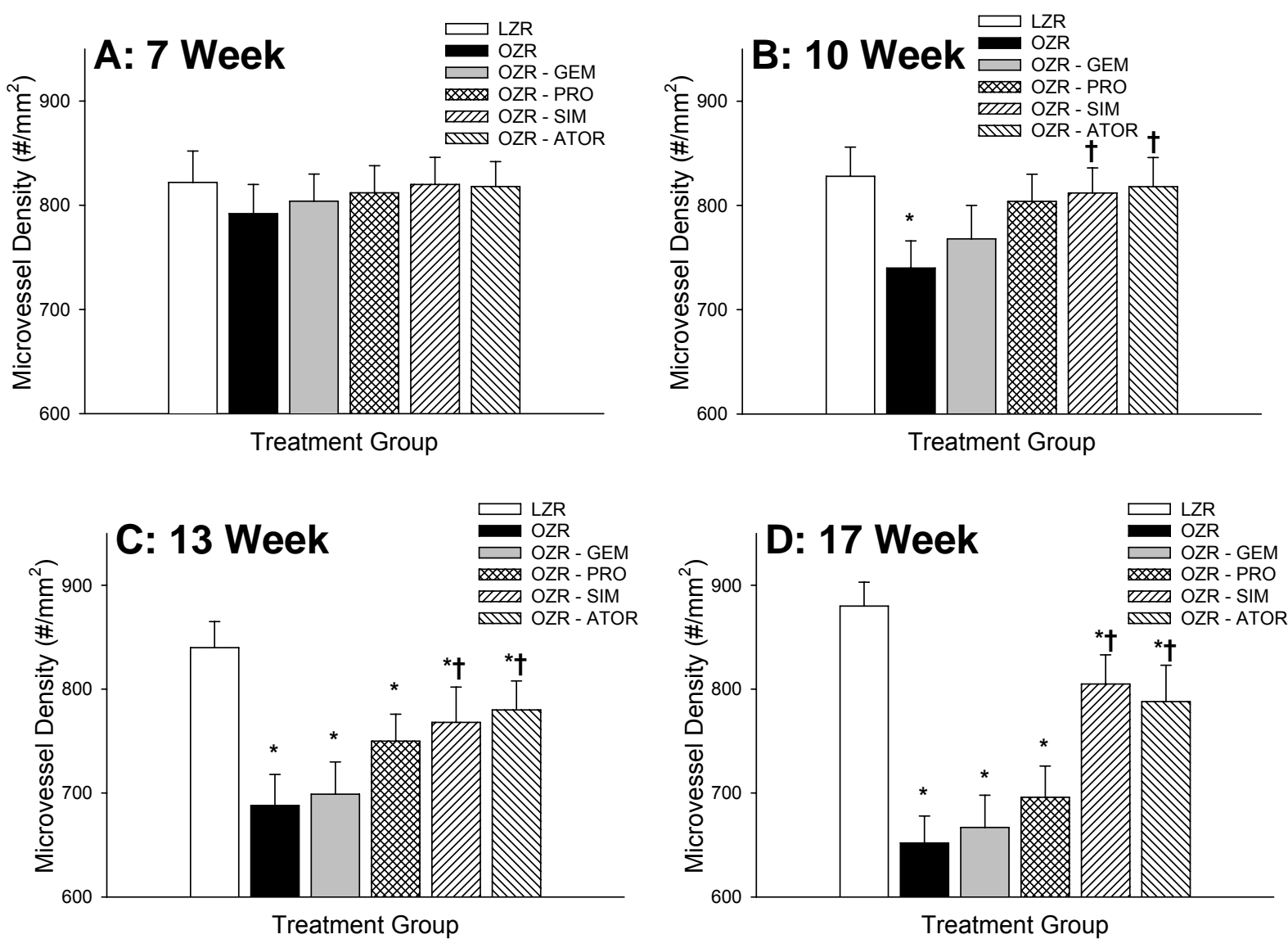
Figure 2.
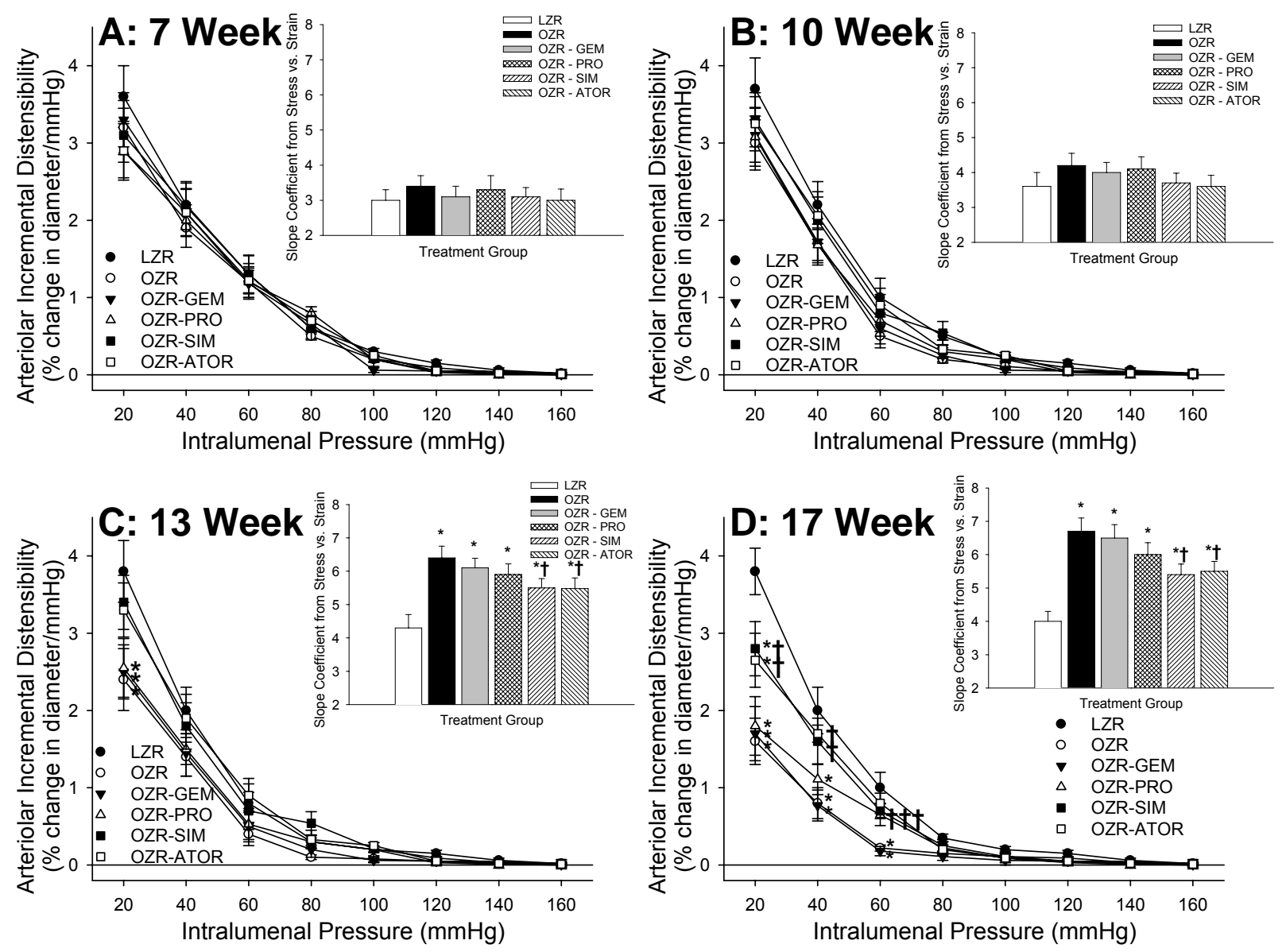


\section{Figure 3.}
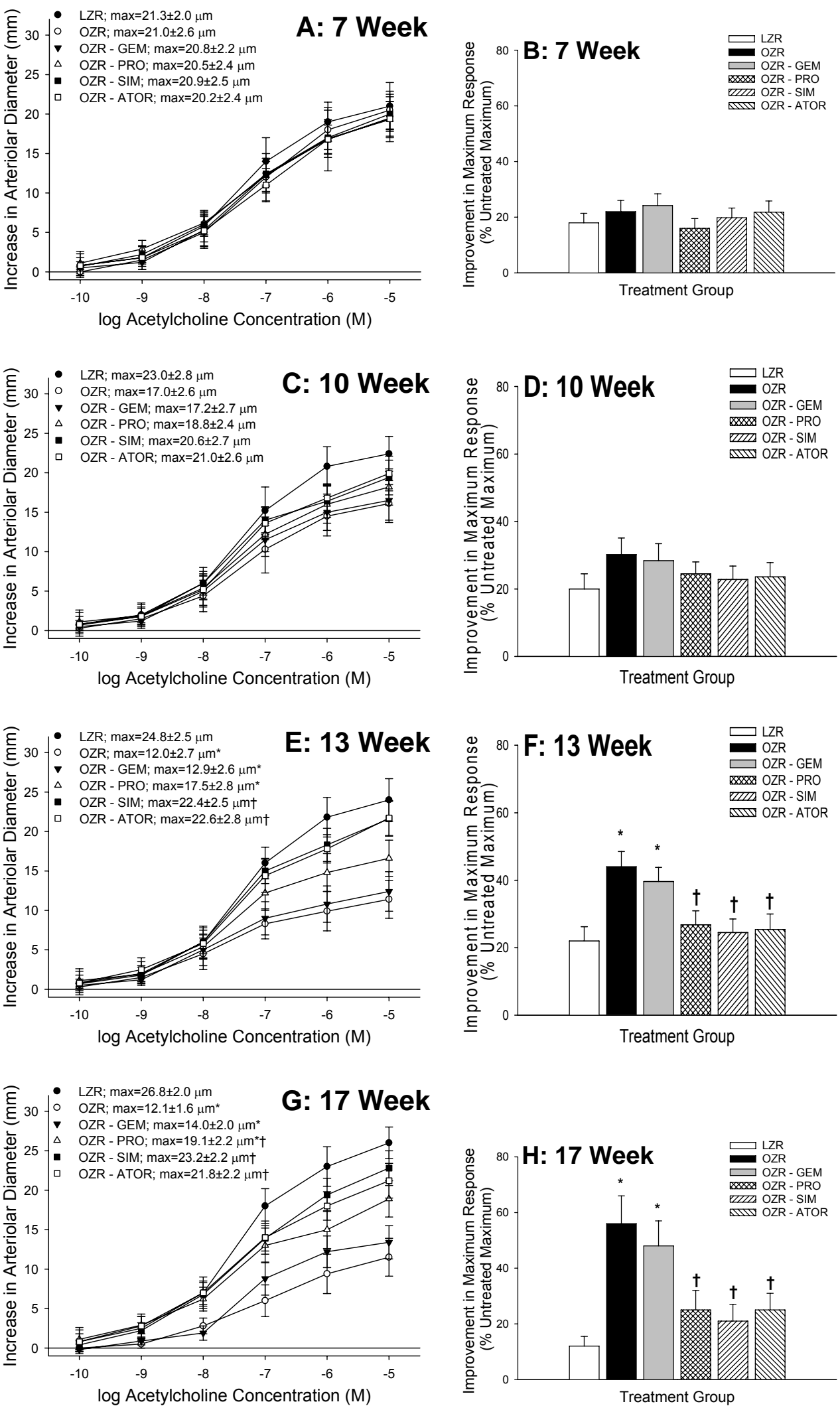
Figure 4.
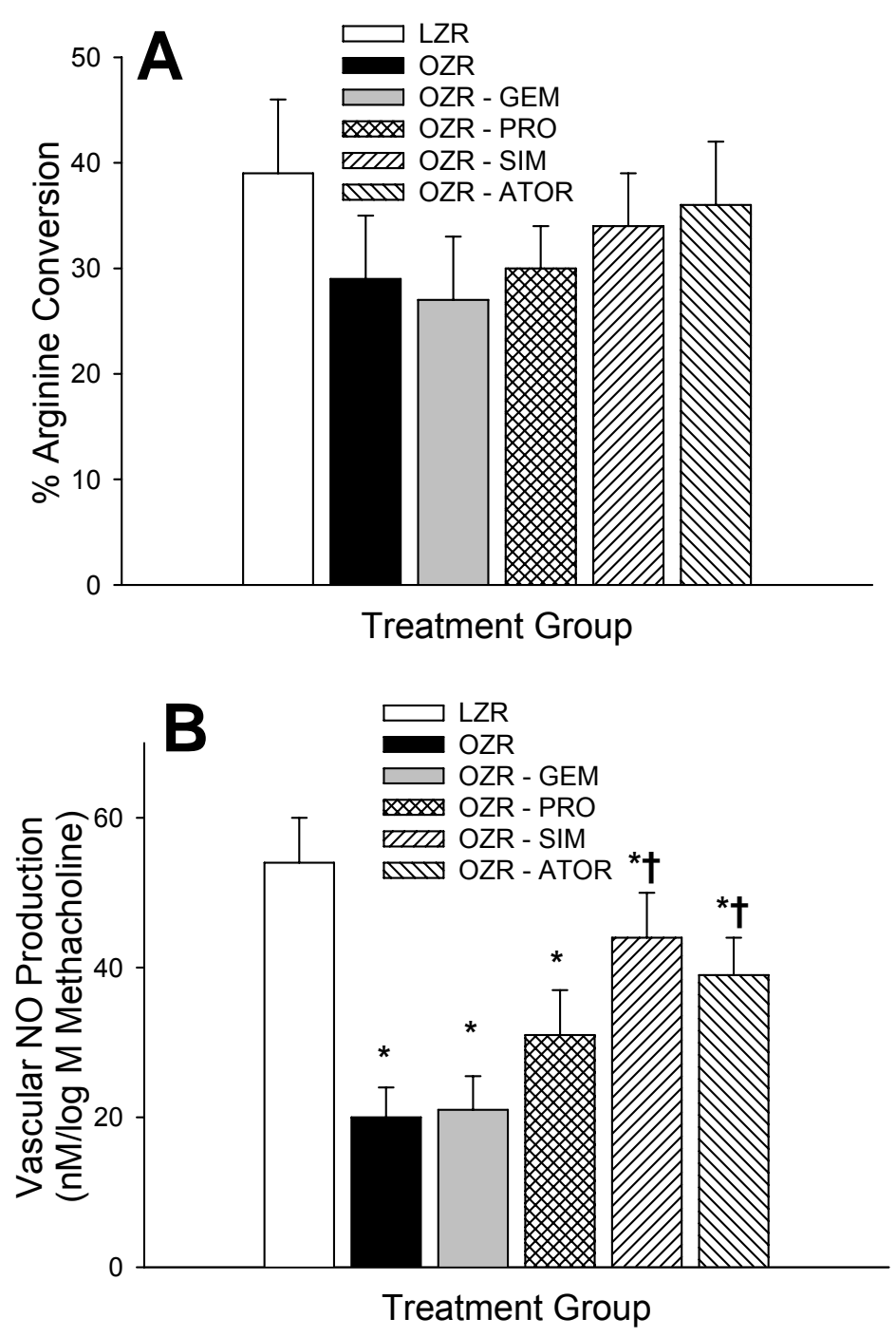


\section{Figure 5.}
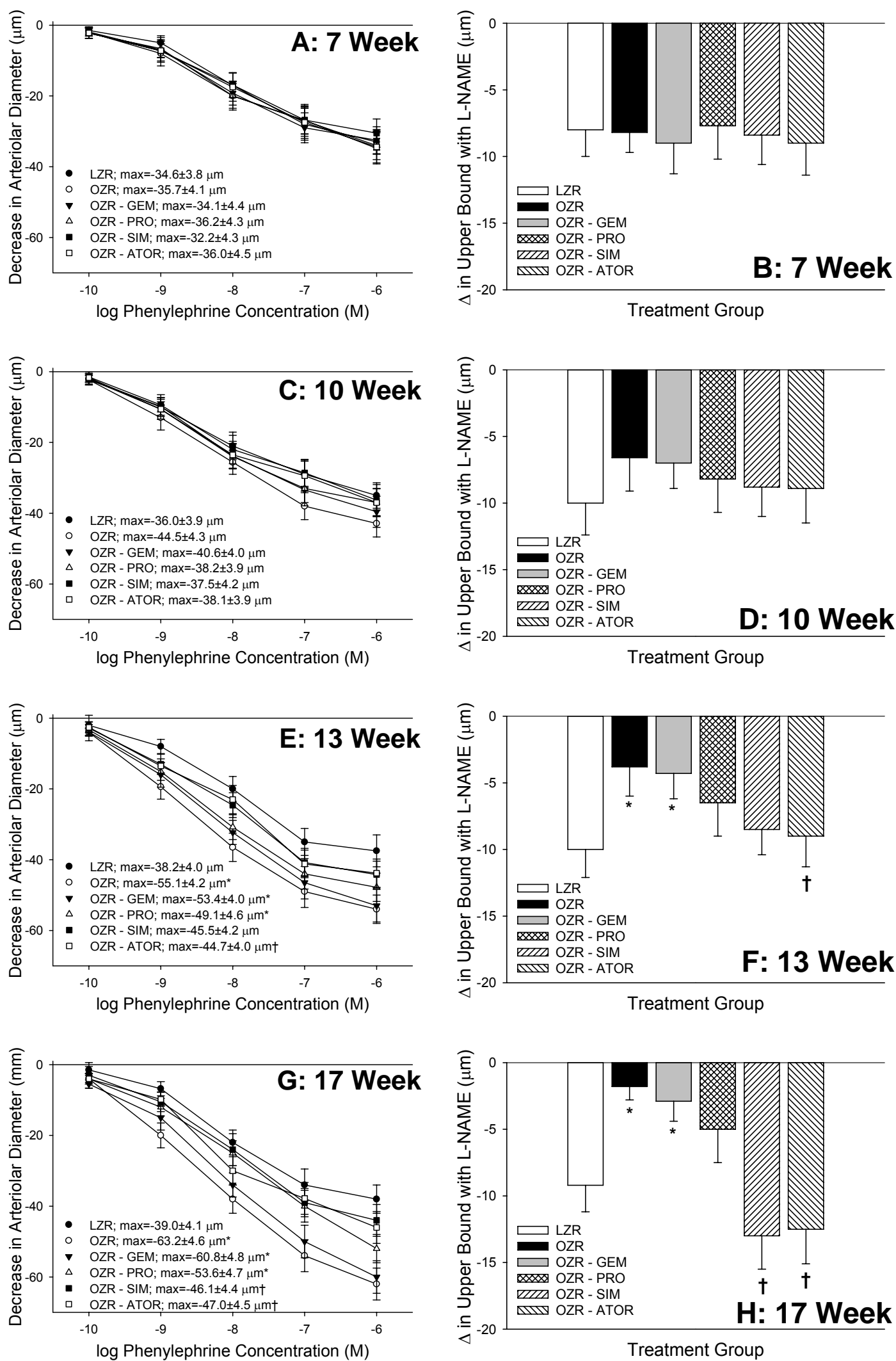
Figure 6.
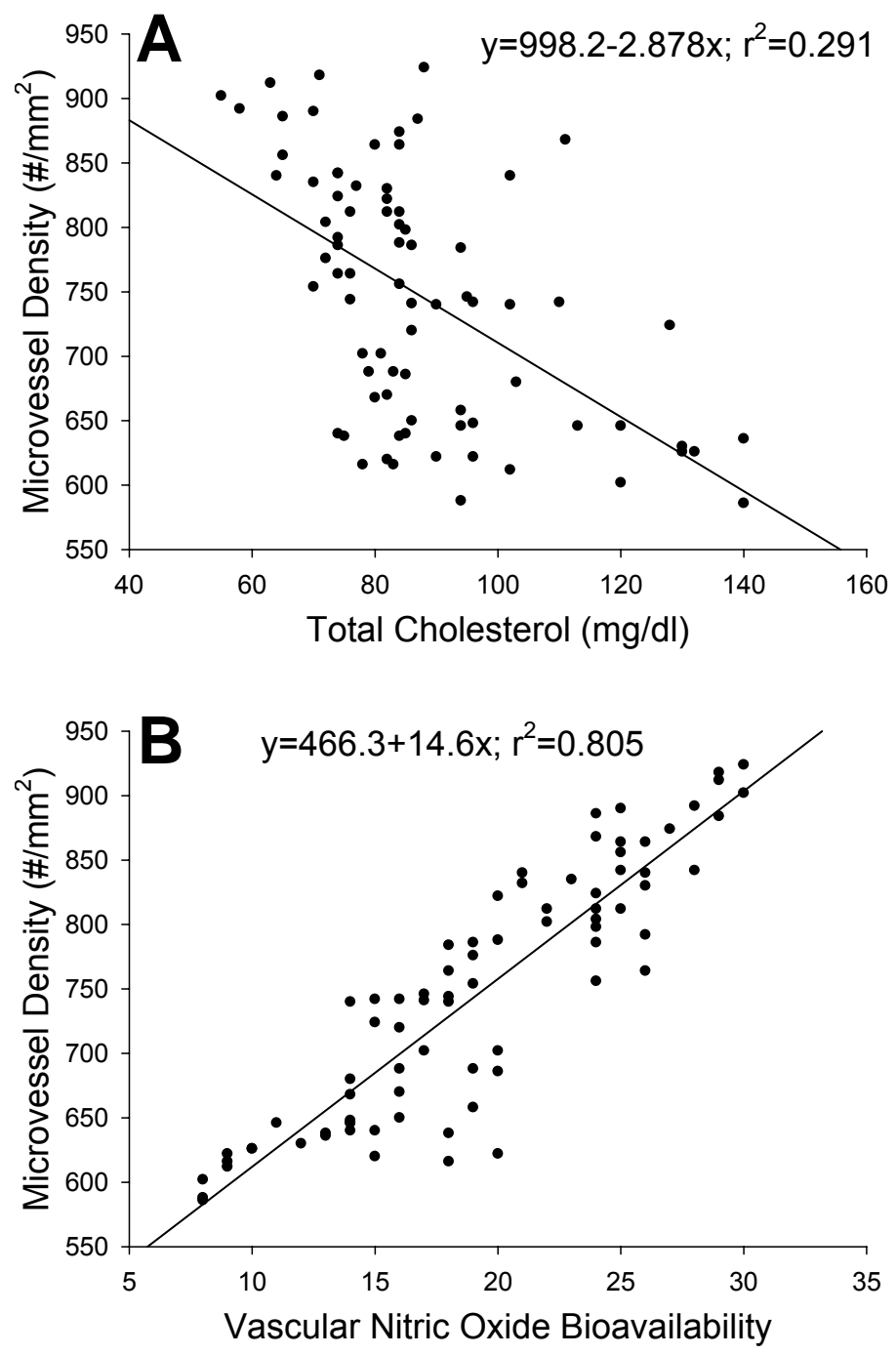
Figure 7.
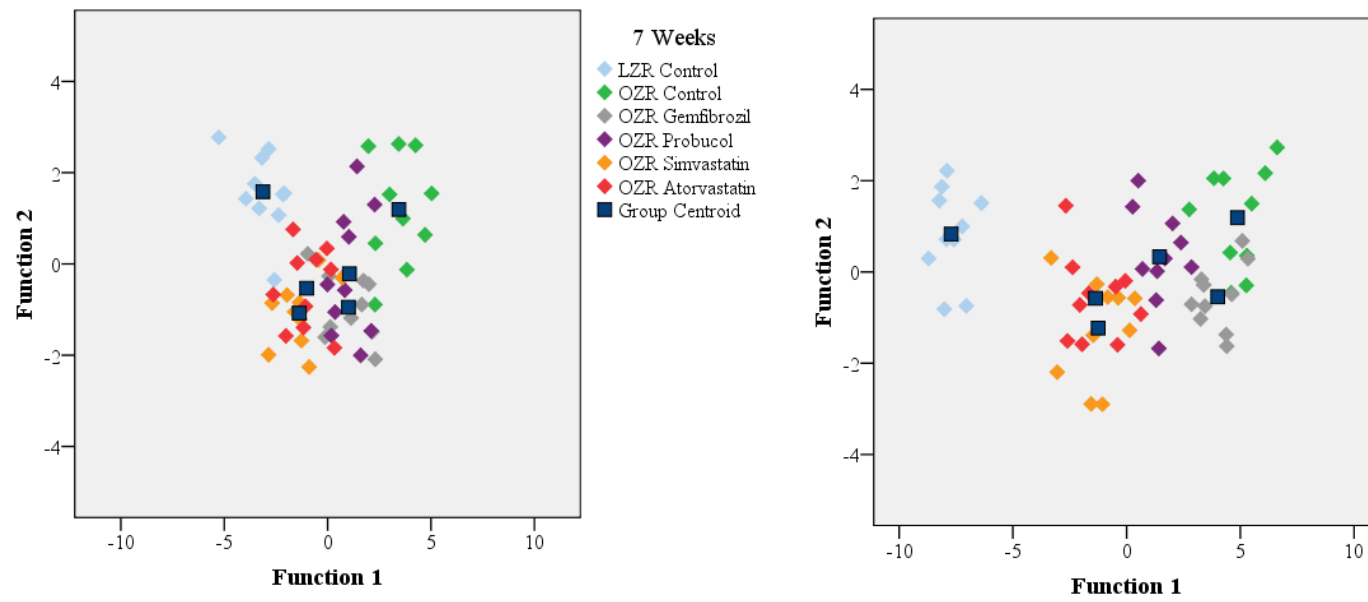

10 Week LZR Control - OZR Control OZR Gemfibrozil OZR Simvastatin OZR Atorvastatin - Group Centroid

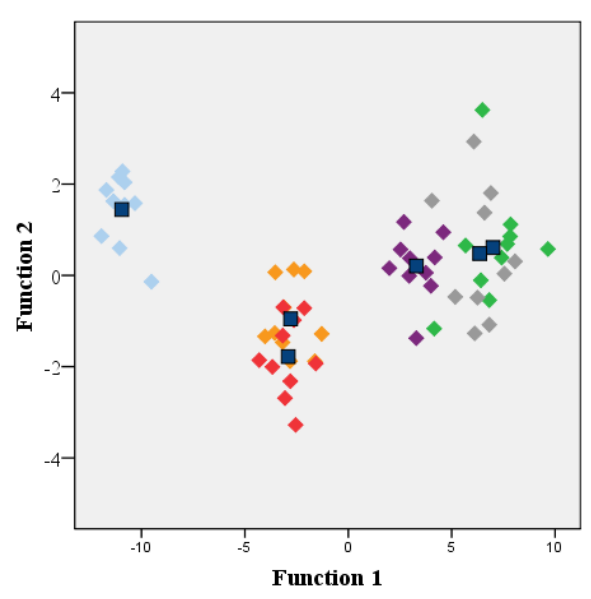

13 Week LZR Control - OZR Control - OZR Gemfibroz OZR Simvastatin - OZR Atorvastatin

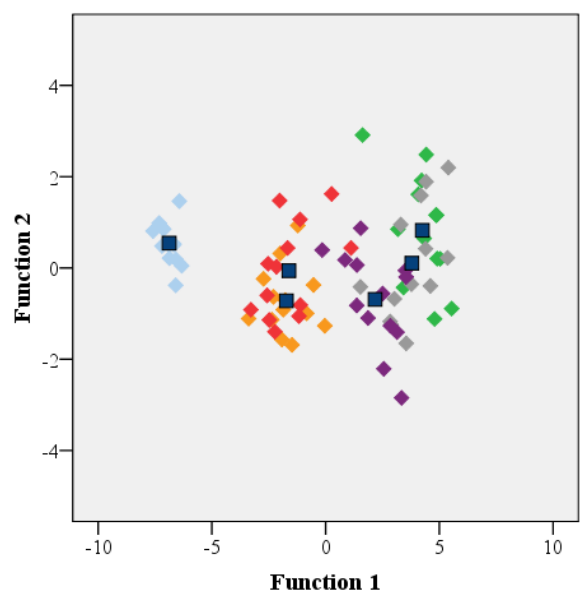

17 Week LZR Control OZR Control OZR Gemfibrozl OZR Simvastatin OZR Atorvastatin

Function 1 
Figure 8.
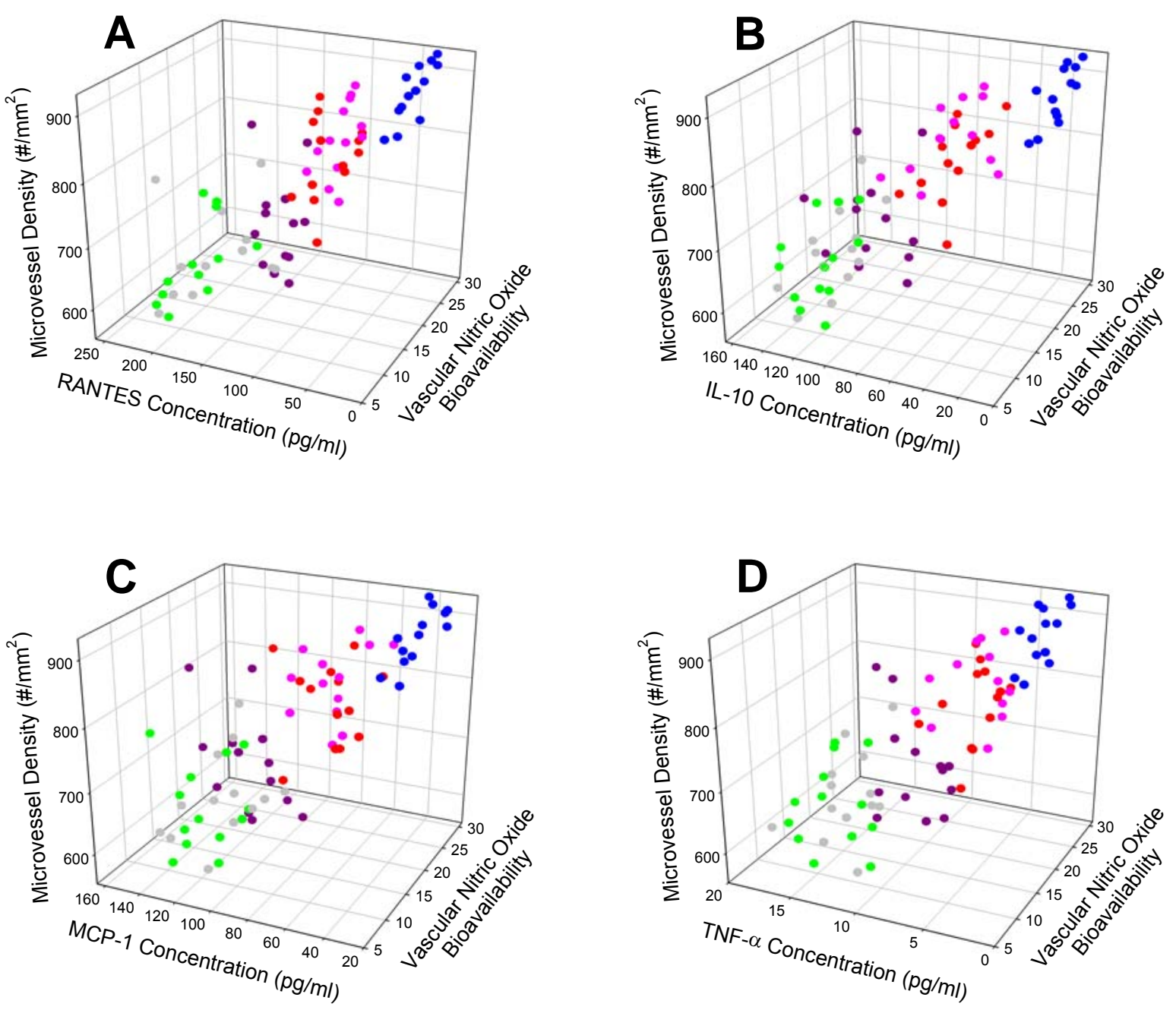


\title{
Chapter 5
}

\section{TEMPORAL PROGRESSION OF MICROVASCULAR RAREFACTION IN THE METABOLIC SYNDROME; THE ROLE OF THROMBOXANE A2}

\author{
Adam G. Goodwill ${ }^{1,2}$ and Jefferson C. Frisbee ${ }^{1,2}$ \\ Center for Cardiovascular and Respiratory Sciences ${ }^{1}$ \\ Departments of Physiology and Pharmacology², \\ West Virginia University School of Medicine, Morgantown, WV 26506
}

Running Head: Biphasic Rarefaction in the Metabolic Syndrome

Key Words: vascular remodeling, vascular reactivity, rodent models of obesity, cyclooxygenase, prostanoids

Support: American Heart Association (SDG 0330194N; EIA 0740129N) and NIH (R01 DK64668). 


\section{ABSTRACT}

The metabolic syndrome represents an integrated metabolic and cardiovascular disorder reflecting the development of multiple risk factors for a poor cardiovascular outcome, including obesity, insulin resistance, dyslipidemia, hypertension and the genesis of pro-inflammatory and pro-oxidant environments. The obese Zucker rat (OZR) represents an excellent model of metabolic syndrome, and develops this condition due to chronic hyperphagia. While impairments to vascular reactivity with metabolic syndrome have been well established and are partly a function of reduced nitric oxide bioavailability and altered arachidonic acid metabolism, we have made the novel and consistent observation that reductions to skeletal muscle microvascular density (rarefaction) also develop in parallel with reactivity alterations, and that this plays a key contributing role in contributing to disparities between matching metabolic demand to perfusion. While our previous publications have demonstrated that the full extent of rarefaction is well predicted by a chronic loss of nitric oxide bioavailability, our most recent data strongly suggest that an early pulse of rarefaction develops very rapidly in the OZR model of metabolic syndrome which significantly precedes any demonstrable loss in NO bioavailability. Our advanced statistical analyses have suggested that the strongest associated parameter with the early pulse of microvascular rarefaction is a shift in vascular arachidonic acid metabolism from prostacyclin $\left(\mathrm{PGI}_{2}\right)$ to thromboxane $A_{2}\left(T x A_{2}\right)$. Presented within this manuscript are data supporting a role for $\mathrm{TxA}_{2}$ elevations in early rarefaction and underlying role for elevations in oxidant stress and TNF $\alpha$ levels in the elevations in TXA2 production. 


\section{INTRODUCTION}

With the development and maintenance of an obese state comes the establishment of pro-oxidant $(22 ; 28 ; 32 ; 40)$, pro-thrombotic $(1 ; 31)$, and proinflammatory $(8 ; 24 ; 26 ; 35)$ conditions along with concurrent development of insulin resistance $(25 ; 34 ; 43)$, dyslipidemia (29), and hypertension (2). This constellation of an initiating pathology of obesity along with its associated co-morbidities is clinically classified as the "metabolic syndrome" (38). It is estimated that as many as 50 million Americans (38) currently fit the requisite criteria for diagnosis of metabolic syndrome with those numbers steadily increasing both nationally (7) and globally (23). While each individual pathology associated with metabolic syndrome is significant, their greatest impact on public health is that they dramatically increase the risk for developing peripheral vascular disease, resulting in an imbalance between skeletal muscle demand and adequate perfusion $(12 ; 13 ; 18 ; 41 ; 42)$. This imbalance in demand:perfusion matching leads to ischemia (15), compromised tissue function (15), diminished tissue viability(37) and quality of life issues (37).

The obese Zucker rat (OZR) serves as an established model of metabolic syndrome, owing to a dysfunctional leptin receptor gene which results in a chronic state of hyperphagia. These animals become rapidly obese and, in turn, develop insulin resistance, dyslipidemia and moderate hypertension along with the pro-thrombotic, proinflammatory and pro-oxidant environment characteristic of their human metabolic syndrome counterparts. Previous studies by multiple investigative groups have demonstrated negative vascular outcomes in OZRs with the development of metabolic syndrome, including alterations to vascular wall mechanics, impairments to multiple 
indexes of dilator reactivity, alterations to signaling mechanisms underlying constrictor reactivity, and a rarefaction of microvascular networks within multiple tissues $(6 ; 15 ; 18$; $19 ; 40 ; 42)$. The culmination of these alterations to microvascular structure and function within OZRs can result in profound impairments to the perfusion of tissue under an array of physiological and pathological conditions.

In recent years, studies have demonstrated that alterations to arachidonic acid metabolism (AA) through the cyclooxygenase (COX) pathway may contribute to the demand:perfusion disparities seen in OZRs. AA is stored in the cell membrane of most resting cells, esterified to glycerol groups of phospholipids (9). It is released from this "stored" esterified state when activated phospholipases (A, C or D) catalyze hydrolysis of the phospholipid thereby allowing for AA desequestration (9). Upon release, this free arachidonate can: be reincorporated into phospholipids, diffuse away from the cell, or diffuse into the cell at which time it is subject to metabolism (30). The metabolism of arachidonic acid is characterized by three major pathways: Cyclooxygenase, Lipoxygenase and Cytochrome P450. Alterations to relative metabolism through each of these pathways has been demonstrated to occur in a number of disease states (3-5; 33). Recent studies have demonstrated a shift in the balance of $\mathrm{TxA}_{2}$ and $P \mathrm{PG}_{2}$ produced downstream from $\mathrm{COX}$ with a predomination of $\mathrm{TXA}_{2}$ production resulting in attenuated dilator responses as the constrictor prostanoid $\mathrm{TxA}_{2}$ blunts the response of the typically predominant COX dilator prostanoid, $\mathrm{PGI}_{2}(20 ; 36 ; 36 ; 40)$.

In addition to the above mentioned vasomotor dysfunction found in metabolic syndrome, there are significant vascular network remodeling components in the skeletal muscles of metabolic syndrome individuals and OZRs. There is a well documented and 
progressive loss of tissue capillarity in metabolic syndrome subjects termed rarefaction $(21 ; 27 ; 39)$. This rarefaction process while well documented, is only poorly understood. Recent work from this lab has attributed much of the rarefactive process to diminished NO bioavailability as therapeutics which spared NO were also able to maintain tissue microvessel density $(10 ; 11 ; 16)$. This study re-examines those earlier findings which focused on tissue microvessel density during a single timepoint and attempts to establish the progression of rarefaction as it parallels development of the metabolic syndrome. Rather than wait for the classically employed 17-week age range wherein OZRs are showing all major symptoms of the metabolic syndrome, this study begins at 7 weeks of age where metabolic syndrome symptoms are intermediate at best. The novel observation of this work is that our understanding of rarefaction is not inaccurate though it is incomplete. There does, indeed, appear to be a NO mediated phase of rarefaction but only subsequent to an early and mechanistically distinct rarefactive process mediated by the obesity associated shift in prostanoid production through the COX pathway.

The purpose of the present study was to identify key contributors to the process of obesity related skeletal muscle microvascular rarefaction in an established animal model of the metabolic syndrome. Based on the existing data set, the hypothesis to be tested was that metabolic syndrome mediated rarefaction is a linear process regulated by a single major mechanism. This somewhat implausible hypothese was rapidly disproven as studies demonstrated that the process of rarefaction is far more complex. Rarefaction in metabolic syndrome is neither linear nor mediated by a single mechanism. The system is regulated by multiple, temporally and mechanistically 
distinct mechanisms with an early rarefactive pulse mediated by shifts in prostanoid production and a later pulse mediated by diminished NO bioavailability.

\section{MATERIALS AND METHODS:}

Animals: Male LZRs and OZRs (6-20 wks old, as indicated; 17 weeks old if not otherwise indicated) were used for all experiments. Rats were fed standard chow and tap water ad libitum. After an overnight fast, rats were anesthetized with injections of pentobarbital sodium (50 mg/kg ip) and received tracheal intubation to facilitate maintenance of a patent airway. In all rats, a carotid artery and an external jugular vein were cannulated for the determination of arterial pressure and for the intravenous infusion of supplemental anesthetic, if necessary. Blood was drawn from the jugular vein to be used for the biochemical determination of plasma glucose (Freestyle, Abbott Diabetes Care, Alameda, CA), insulin (Millipore, St. Charles, MO), and measurements for TNFa (Millipore, St. Charles MO) as well as a plasma lipid profile (Stanbio, Boerne, TX) and nitrotyrosine (Oxis, Foster City, CA) from each animal. Chronic TNF $\alpha$ inhibition was accomplished in a subset of these animals through daily i.p. injection of the TNF $\alpha$ inhibitor pentoxifylline $[30 \mathrm{mg} / \mathrm{kg} / \mathrm{day}]$. In additional subsets of animals oxidant scavenging was achieved through the use of the membrane permeable superoxide dismutase mimetic 1-Oxyl-2,2,6,6-tetramethyl-4-hydroxypiperidine (TEMPOL) $\left[10^{-3} \mathrm{M}\right.$ in drinking water]. In all animals, water consumption was monitored daily to assure that dosing was within expected parameters.

Histological Determination of Microvessel Density: With the rat under anesthesia, the right gastrocnemius muscle was removed, rinsed in PSS, and lightly fixed in $1 \%$ formalin. Muscles were then embedded in paraffin and cut into $5-\mu \mathrm{m}$ cross sections, and 
stained for Griffonia simplicifolia I lectin (Sigma). From each gastrocnemius muscle, six randomly selected individual cross sections were used for analysis, with six randomly selected regions within an individual cross section chosen for study. Each region of study had an area of $\sim 1.47 \times 10^{5} \mu \mathrm{m}^{2}$. After staining, sections were rinsed three times in PSS and mounted on microscope slides with a water-soluble mounting medium (SP, ACCU-MOUNT 280, Baxter). With the use of epifluorescence microscopy, localization of lectin stained microvessels was performed with a Nikon Eclipse 80i upright microscope with a X20 objective lens (Plan Fluo phase, numerical aperture 0.5). Excitation was provided by a 75-W xenon arc lamp through a Lambda 10-2 optical filter changer (Sutter Instruments; Novato, CA) controlling a 595-nm excitation filter and 615$\mathrm{nm}$ emission filter. The microscope is coupled to a cooled charge-coupled device camera (Photometrics CoolSNAP ES).

Determination of Vascular TxA ${ }_{2}$ Production: Vascular production of 11-dehydrothromboxane $\mathrm{B}_{2}$ [the stable plasma breakdown product of $\mathrm{TxA}_{2}$ ] was assessed using pooled conduit arteries (femoral, saphenous, and iliac arteries) from LZRs and OZRs (treated \& control). Pooled vessels from each animal were incubated in microcentrifuge tubes in $1 \mathrm{ml}$ PSS for 30 min under control conditions $\left(21 \% \mathrm{O}_{2}\right)$ at room temperature and stimulated with an addition of exogenous arachidonic acid $\left(10^{-5} \mathrm{M}\right)$. After the 30 -min period, this PSS was transferred to a fresh tube, frozen in liquid $\mathrm{N}_{2}$, and stored at $-80^{\circ} \mathrm{C}$. Metabolite release by the vessels was determined using commercially available EIA kits for 11-dehydro- $\mathrm{TxB}_{2}$ (Cayman Chemicals). 
Determination of Vascular Nitric Oxide Bioavailability: From each animal, the abdominal aorta was removed and vascular NO production was assessed using amperometric sensors (World Precision Instruments). Briefly, aortae were isolated, sectioned longitudinally, pinned in a silastic coated dish, and superfused with warmed $(37 \circ \mathrm{C})$ PSS equilibrated with $95 \% \mathrm{O} 2$ and $5 \%$ CO2. The NO sensor (ISO-NOPF 100) was placed in close apposition to the endothelial surface and a baseline level of current was obtained. Subsequently, increasing concentrations of methacholine $\left(10^{-10}-10^{-6} \mathrm{M}\right)$ were added to the bath and the changes in current were determined. To verify that the recorded data represented endothelium-dependent NO release, responses were reevaluated following acute treatment of the aortae with L-NAME $\left(10^{-4} \mathrm{M}\right)$.

Statistical Analyses: As this work is preliminary, each group contains 3-4 animals. As such, the study is too insufficiently powered to provide statistical analyses at this time. Ongoing work will, ultimately, provide greater power allowing for the employment of appropriate statistical methods. The notable exception to this occurs in Table 1 which draws on a more robust data set allowing for animal groups of 8 .

\section{RESULTS}

Baseline characteristics of lean and obese Zucker rats are presented in Table 1. The OZR shows significantly increased mass as early as 6 weeks of age, when compared to age matched lean counterparts, with the disparity in lean vs. obese mass only becoming more pronounced with time. Additionally, by 6 weeks of age, obese Zucker rats clearly demonstrate hyperinsulemia as well as hypertriglyceridemia. By 12 weeks of age, the obese Zucker rat additionally manifests hyperglycemia with demonstrable hypercholesterolemia finally manifesting at 17 weeks of age. 
Owing to the fact that the obese Zucker rat demonstrates all measured metrics of metabolic syndrome employed in this study by 17 weeks of age, initial measures of the effects of metabolic syndrome on tissue capillarity were performed within this age window. Results of these analyses are demonstrated in Figure 1A which clearly demonstrates a nearly $30 \%$ loss of microvessel density (rarefaction) when comparing the gastrocnemius muscle microvessel density in 17 week old lean and obese Zucker rats. Acknowledging that the metabolic syndrome is a process that develops over time in the Zucker rat and supported by the evidence shown in Table 1, further analyses of the temporal effects of metabolic syndrome on skeletal muscle microvessel density were performed from 7 to 20 weeks of age in approximately 3 week iterations. The results of these analyses, summarized in Figure 1B, demonstrate that at 7 weeks of age, obese Zucker rats maintain normal tissue capillarity despite the elevated mass, plasma insulin and triglyceride levels demonstrated in Table 1. However, given time, these rats undergo a biphasic rarefactive process wherein there is an early period of rarefaction from 7 to 10 weeks of age constituting an approximate $15 \%$ loss of microvessel density in the gastrocnemius muscle. This early rarefaction is followed by a plateau from 10-13 weeks of age wherein there is no apparent loss of microvessel density. Subsequent to the rarefactive plateau is a late phase of rarefaction accountable for the remaining $15 \%$ loss of microvessel density. Analyses to 20 weeks of age and beyond (data not shown) demonstrate that this capillarity remains relatively constant through the remainder of the animal's life.

Previous studies from this laboratory have demonstrated an inverse relationship between vascular NO bioavailability and skeletal muscle microvessel density in the 
Zucker rat $(17 ; 18)$. Since these studies were performed in the 17 week age with no attention to the temporal contribution, and owing to the data in Figure 1B demonstrating a biphasic phenomenon, vascular NO bioavailability was assayed across the 7 to 20 week age time-course of the study. Figure $2 \mathrm{~A}$ clearly demonstrates significant impairment to vascular NO bioavailability beginning at 13 weeks of age with more pronounced impairment at later time-points. This coincides well with late rarefaction, while removing NO bioavailability as a likely causative agent in early rarefaction. Based on previous work in this lab which demonstrated impairments in COX mediated prostanoid balances in the obese Zucker rat, favoring a production of $\mathrm{TxA}_{2}$ over prostacyclin (19), stimulated vascular production of $\mathrm{TxA}_{2}$ was measured in all time points. Figure $2 \mathrm{~B}$, which summarizes the results of these analyses, demonstrates that vascular production of 11-dehydro-thromboxane-B2, the stable breakdown product of $\mathrm{TxA}_{2}$ is drastically elevated across the period of early rarefaction and remains so through 20 weeks of age.

Determination of a mechanistic linkage between elevations in $\mathrm{TxA}_{2}$ production and early rarefaction was accomplished by competitively antagonizing the $\mathrm{TxA}_{2}$ receptor, TP, with the compound SQ-29548. Figure 3A shows the results of this study wherein early rarefaction was abolished in obese Zucker rats via treatment with SQ29548 with no apparent non-specific effects of the compound on lean control animals. Figure $3 B$ shows the results of an early withdrawal study where animals received SQ29548 from 6 to 13 weeks of age, however, at 13 weeks of age treatment was discontinued. The results of this portion of the study demonstrate that rarefaction begins anew immediately following discontinuation of TP receptor antagonism. 
Figure 4 summarizes the data related to determining the mechanistic underpinnings of obesity associated elevations in $\mathrm{TxA}_{2}$. Figures $4 \mathrm{~A}$ and $4 \mathrm{~B}$ demonstrate drastic elevations in systemic oxidant stress and plasma TNF $\alpha$ concentrations, respectively, with increasing duration of the metabolic syndrome. In both the case of oxidant stress and inflammation, elevations occur early and levels of both nitrotyrosine and $\mathrm{TNF} \alpha$ remain elevated through the time-course in untreated obese Zucker rats. Elevations in oxidant stress were diminished via treatment with the membrane permeable superoxide dismutase mimetic TEMPOL while production of TNF $\alpha$ was inhibited through the use of pentoxifylline. The effects of these inhibitions on stimulated vascular $\mathrm{TxA}_{2}$ production is summarized in Figure 4 panels $\mathrm{C}$ and $\mathrm{D}$. Chronic diminishment of superoxide levels resulted in an intermediate production of $\mathrm{TxA}_{2}$ across the time-course. This same effect was seen in the case of TNF $\alpha$ synthesis inhibition where chronic pentoxifylline treatment was able to maintain intermediate levels of $\mathrm{TxA}_{2}$. The phenotypic consequences of chronic inhibition of oxidant stress mechanisms or TNF $\alpha$ mediated inflammatory mechanisms are summarized in Figure 4 panels $E$ and $F$. Panel $E$ demonstrates that chronic inhibition of superoxide mediated effects is sufficient to blunt but not abolish the rarefactive process demonstrated with continued duration of metabolic syndrome in obese Zucker rats. In contrast, Panel F demonstrates that inhibition of the inflammatory mediator TNF $\alpha$ with pentoxifylline is sufficient to abolish rarefaction in obese Zucker rats through 17 weeks of age.

\section{DISCUSSION}


With the development of metabolic syndrome comes the inevitable loss of ability to match demand and perfusion in peripheral tissues $(12 ; 14)$. Numerous investigations have demonstrated that alterations to vasomotor function significantly contribute to the inability to properly regulate perfusion into these tissues. However, even when the influences of vasomotor alterations are systematically removed, perfusion levels still remain impaired with increasing metabolic demand (10). This impairment is attributed to a bulk loss of microvessels/capillaries in these peripheral tissues, a process termed rarefaction.

Despite the phenomenon of rarefaction being clinically and experimentally well established, the mechanistic underpinnings of the process are poorly understood. Previous work has demonstrated that capillary losses parallel loss in nitric oxide bioavailability and with experimental evidence to support the rationale that the loss in tissue capillarity is a direct result of diminished NO bioavailability $(11 ; 18)$. These previous studies have provided tremendous insight into the mechanisms leading to rarefaction and resulting in perfusion demand disparities but have suffered from the lack of an adequate temporal component.

As Table 1 in this study demonstrates, the development of metabolic syndrome occurs along a temporal continuum with some aspects preceding others by necessity. For instance, obese Zucker rats manifest demonstrable hyperinsulemia as early as 6 weeks of age. This hyperinsulemia must, by necessity precede any notion of the animal being a model of type II diabetes as glycemic control remains unimpaired until 12 weeks of age. By logical extension, the fact that the animal suffers from fewer comorbities than those found at later time-points in the disease could have significant impact on the 
effects of the metabolic syndrome on skeletal muscle microvessel density. Though this distinction seems trivial in an animal that is able to fully manifest a metabolic syndrome phenotype by 17 weeks of age, the implications in humans are far more significant as development of metabolic syndrome is a much more gradual process typically taking years or even decades to fully manifest.

The most obvious and significant finding of this study is found in Figure 1 panel B which highlights the biphasic nature of obesity related microvascular rarefaction. Prior to the onset of a type II diabetic phenotype, in the 7-10 week age window, a rarefactive pulse constituting $\sim 50 \%$ of the total microvessel loss occurs. It is not until 13 weeks of age that a second rarefactive pulse occurs resulting in all remaining rarefaction. As this window is subsequent to the development of diabetes at approximately 12 weeks of age, the second rarefaction highlights the notion that as the pathologies evolve so do the consequences of those pathologies. An admitted limitation of this study is the lack of attempt to correct the diabetes in these animals to determine its contributions to later rarefaction. What does become evident even in these early figures, however, is that microvascular rarefaction in the metabolic syndrome is not the simple linear process that it is often portrayed to be.

The logical extension of a biphasic process is that each phase may be mediated through distinct processes. Data supporting this hypothesis are presented in Figure 2 which shows that the loss of NO bioavailability occurs in obese Zucker rats subsequent to the early rarefactive period precluding the possibility of NO reductions being a causative agent in the early microvessel loss. Building on data from previous studies which demonstrated a shift in vascular prostanoid production with obesity, Figure 2 
panel $\mathrm{B}$ presents the possibility of elevations in $\mathrm{TxA}_{2}$ as a causative factor in early rarefaction. This correlative data are then further extended to demonstrate causality as antagonism of the TxA2 signaling pathway results in total ablation of early rarefaction (Figure 3). Perhaps more interesting was the discovery that when antagonism of the TxA2 pathway was discontinued, rarefaction was renewed but blunted as indicated by the diminished slope of rarefaction in 13-17 week window. While further studies are required to determine what mediates this post-therapeutic rarefaction, the 13-17 week blunting would strongly indicate that early TxA2 elevations are either necessary for later rarefaction and have some sort of causative relationship to NO bioavailability reductions or the TxA2 elevations somehow exacerbate the effects of NO bioavailability reductions enhancing the consequence of those reductions with regards to rarefaction. Whatever the case may be, the evidence provided by these data indicates that while the two phases of rarefaction are mechanistically distinct, they may also be interconnected.

The final figures of this study attempt to demonstrate the cause of these elevations in TxA2 production with obesity. A significant consequence early in the metabolic syndrome is the development of a pro-inflammatory and pro-oxidant environment. As increased oxidative stress and inflammation is able to activate numerous signaling pathways, they serve as logical targets of investigation for changes in the production of essentially any vascular metabolite. Additionally, work from our lab and others has implicated elevations in superoxide and TNFa, specifically, in alterations to TxA2 production. Figure 4 clearly demonstrates that both superoxide and TNFa elevations contribute to elevations in vascular TxA2 levels with the phenotypic consequence of enhanced rarefaction. 
Taken together, these data demonstrate that metabolic syndrome related microvascular rarefaction is a biphasic process in which each phase is both temporally and mechanistically distinct. Early rarefaction is mediated by elevations in vascular TxA2 levels whereas late rarefaction is mediated by diminished vascular NO bioavailability. Preliminary evidence would indicate that the later phase of rarefaction is at least partially enhanced, if not totally dependent, on early rarefactive processes. Additionally, these early TxA2 mediated rarefactive processes are the result of elevations in systemic oxidant stress and systemic inflammation. Taken together these data highlight the TxA2 pathway as a target for potential future investigations into therapeutic treatments for metabolic syndrome related skeletal muscle microvascular rarefaction. Additionally this study supports the idea of prophylactic treatment of metabolic syndrome subjects with anti-inflammatory and anti-oxidant drugs as this study provides another example of an instance where pathophysiologic elevations in either of those two systems is deleterious to cardiovascular outcome. 


\section{Reference List}

1. Ageno W, Dentali F and Grandi AM. New evidence on the potential role of the metabolic syndrome as a risk factor for venous thromboembolism. $J$ Thromb Haemost 7: 736-738, 2009.

2. Antic $\mathrm{V}$, Dulloo A and Montani JP. Multiple mechanisms involved in obesityinduced hypertension. Heart Lung Circ 12: 84-93, 2003.

3. Back M. Inhibitors of the 5-lipoxygenase pathway in atherosclerosis. Curr Pharm Des 15: 3116-3132, 2009.

4. Bazinet RP. Is the brain arachidonic acid cascade a common target of drugs used to manage bipolar disorder? Biochem Soc Trans 37: 1104-1109, 2009.

5. Bhattacharya S, Mathew G, Jayne DG, Pelengaris S and Khan M. 15lipoxygenase-1 in colorectal cancer: a review. Tumour Biol 30: 185-199, 2009.

6. Bray GA. The Zucker-fatty rat: a review. Fed Proc 36: 148-153, 1977.

7. Ervin RB.2009 Prevalence of Metabolic Syndrome Among Adults 20 Years of Age and Over, by Sex, Age, Race and Ethnicity, and Body Mass Index: United States, 2003-2006 [Online]. U.S. Department of Health and Human Services 
Centers for Disease Control and Prevention National Center for Health Statistics, 2009.

8. Fantuzzi G. Adipose tissue, adipokines, and inflammation. J Allergy Clin Immunol 115: 911-919, 2005.

9. Ford DA and Gross RW. Plasmenylethanolamine is the major storage depot for arachidonic acid in rabbit vascular smooth muscle and is rapidly hydrolyzed after angiotensin II stimulation. Proc Natl Acad Sci U S A 86: 3479-3483, 1989.

10. Frisbee JC. Remodeling of the skeletal muscle microcirculation increases resistance to perfusion in obese Zucker rats. Am J Physiol Heart Circ Physiol 285: H104-H111, 2003.

11. Frisbee JC. Reduced nitric oxide bioavailability contributes to skeletal muscle microvessel rarefaction in the metabolic syndrome. Am J Physiol Regul Integr Comp Physiol 289: R307-R316, 2005.

12. Frisbee JC. Impaired skeletal muscle perfusion in obese Zucker rats. Am J Physiol Regul Integr Comp Physiol 285: R1124-R1134, 2003.

13. Frisbee JC. Enhanced arteriolar alpha-adrenergic constriction impairs dilator responses and skeletal muscle perfusion in obese Zucker rats. J Appl Physiol 97: 764-772, 2004. 
14. Frisbee JC and Delp MD. Vascular function in the metabolic syndrome and the effects on skeletal muscle perfusion: lessons from the obese Zucker rat. Essays Biochem 42: 145-161, 2006.

15. Frisbee JC, Hollander JM, Brock RW, Yu HG and Boegehold MA. Integration of skeletal muscle resistance arteriolar reactivity for perfusion responses in the metabolic syndrome. Am J Physiol Regul Integr Comp Physiol 296: R1771R1782, 2009.

16. Frisbee JC, Samora JB, Peterson J and Bryner R. Exercise training blunts microvascular rarefaction in the metabolic syndrome. Am J Physiol Heart Circ Physiol 291: H2483-H2492, 2006.

17. Frisbee JC, Samora JB, Peterson J and Bryner R. Exercise training blunts microvascular rarefaction in the metabolic syndrome. Am J Physiol Heart Circ Physiol 291: H2483-H2492, 2006.

18. Goodwill AG, Frisbee SJ, Stapleton PA, James ME and Frisbee JC. Impact of chronic anticholesterol therapy on development of microvascular rarefaction in the metabolic syndrome. Microcirculation 16: 667-684, 2009.

19. Goodwill AG, James ME and Frisbee JC. Increased vascular thromboxane generation impairs dilation of skeletal muscle arterioles of obese Zucker rats with 
reduced oxygen tension. Am J Physiol Heart Circ Physiol 295: H1522-H1528, 2008.

20. Goodwill AG, Stapleton PA, James ME, D'Audiffret AC and Frisbee JC. Increased arachidonic acid-induced thromboxane generation impairs skeletal muscle arteriolar dilation with genetic dyslipidemia. Microcirculation 15: 621-631, 2008.

21. Hayden MR, Yang Y, Habibi J, Bagree SV and Sowers JR. Pericytopathy: oxidative stress and impaired cellular longevity in the pancreas and skeletal muscle in metabolic syndrome and type 2 diabetes. Oxid Med Cell Longev 3: 290-303, 2010.

22. Holvoet P. Relations between metabolic syndrome, oxidative stress and inflammation and cardiovascular disease. Verh K Acad Geneeskd Belg 70: 193219, 2008.

23. James PT, Leach R, Kalamara E and Shayeghi M. The worldwide obesity epidemic. Obes Res 9 Suppl 4: 228S-233S, 2001.

24. Knudson JD, Dick GM and Tune JD. Adipokines and coronary vasomotor dysfunction. Exp Biol Med (Maywood) 232: 727-736, 2007. 
25. Kolka CM, Harrison LN, Lottati M, Chiu JD, Kirkman EL and Bergman RN. Dietinduced obesity prevents interstitial dispersion of insulin in skeletal muscle. Diabetes 2009.

26. Lee YH and Pratley RE. The evolving role of inflammation in obesity and the metabolic syndrome. Curr Diab Rep 5: 70-75, 2005.

27. Lind $L$ and Lithell $H$. Decreased peripheral blood flow in the pathogenesis of the metabolic syndrome comprising hypertension, hyperlipidemia, and hyperinsulinemia. Am Heart J 125: 1494-1497, 1993.

28. Marchesi C, Ebrahimian T, Angulo O, Paradis P and Schiffrin EL. Endothelial nitric oxide synthase uncoupling and perivascular adipose oxidative stress and inflammation contribute to vascular dysfunction in a rodent model of metabolic syndrome. Hypertension 54: 1384-1392, 2009.

29. Marotta T, Russo BF and Ferrara LA. Triglyceride-to-HDL-cholesterol Ratio and Metabolic Syndrome as Contributors to Cardiovascular Risk in Overweight Patients. Obesity (Silver Spring) 2009.

30. Needleman P, Turk J, Jakschik BA, Morrison AR and Lefkowith JB. Arachidonic acid metabolism. Annu Rev Biochem 55: 69-102, 1986. 
31. Paul W, Queen LR, Page CP and Ferro A. Increased platelet aggregation in vivo in the Zucker Diabetic Fatty rat: differences from the streptozotocin diabetic rat. Br J Pharmacol 150: 105-111, 2007.

32. Rafikova O, Salah EM and Tofovic SP. Renal and metabolic effects of tempol in obese ZSF1 rats--distinct role for superoxide and hydrogen peroxide in diabetic renal injury. Metabolism 57: 1434-1444, 2008.

33. Rinaldi S, Landucci F and De Gaudio AR. Antioxidant therapy in critically septic patients. Curr Drug Targets 10: 872-880, 2009.

34. Scaglione R, Di CT, Cariello T and Licata G. Visceral obesity and metabolic syndrome: two faces of the same medal? Intern Emerg Med 2009.

35. Singer $G$ and Granger DN. Inflammatory responses underlying the microvascular dysfunction associated with obesity and insulin resistance. Microcirculation 14: 375-387, 2007.

36. Stapleton PA, Goodwill AG, James ME and Frisbee JC. Altered mechanisms of endothelium-dependent dilation in skeletal muscle arterioles with genetic hypercholesterolemia. Am J Physiol Regul Integr Comp Physiol 293: R1110R1119, 2007. 
37. Stapleton PA, James ME, Goodwill AG and Frisbee JC. Obesity and vascular dysfunction. Pathophysiology 15: 79-89, 2008.

38. The American Heart Association.2009 What is the metabolic syndrome? [Online]. 2009.

39. Toblli JE, Cao G, DeRosa G, Di GF and Forcada P. Angiotensin-converting enzyme inhibition and angiogenesis in myocardium of obese Zucker rats. Am J Hypertens 17: 172-180, 2004.

40. Xiang L, Dearman J, Abram SR, Carter C and Hester RL. Insulin resistance and impaired functional vasodilation in obese Zucker rats. Am J Physiol Heart Circ Physiol 294: H1658-H1666, 2008.

41. Xiang L, Naik JS, Abram SR and Hester RL. Chronic hyperglycemia impairs functional vasodilation via increasing thromboxane-receptor-mediated vasoconstriction. Am J Physiol Heart Circ Physiol 292: H231-H236, 2007.

42. Xiang L, Naik JS, Hodnett BL and Hester RL. Altered arachidonic acid metabolism impairs functional vasodilation in metabolic syndrome. Am J Physiol Regul Integr Comp Physiol 290: R134-R138, 2006.

43. Yang L, Samarasinghe YP, Kane P, Amiel SA and Aylwin SJ. Visceral adiposity is closely correlated with neck circumference and represents a significant 
indicator of insulin resistance in WHO grade III obesity. Clin Endocrinol (Oxf) 2009. 
Table 1

\begin{tabular}{|c|c|c|c|c|c|}
\hline \multicolumn{6}{|c|}{ Lean Zucker Rats } \\
\hline & 6 Weeks & 9 Weeks & 12 Weeks & 15-17 Weeks & 20 Weeks \\
\hline Mass (g) & $142+8$ & $202+10$ & $270+11$ & $364+12$ & $385+13$ \\
\hline Mean Arterial Pressure $(\mathrm{mmHg})$ & $89+4$ & $105+4$ & $101+5$ & $105+4$ & $109+6$ \\
\hline$[\text { Glucose }]_{\text {blood }}(\mathrm{mg} / \mathrm{dl})$ & $106+14$ & $111 \pm 10$ & $106 \pm 14$ & $121 \pm 13$ & $125+14$ \\
\hline$[\text { Insulin] }]_{\text {plasma }}(\mathrm{ng} / \mathrm{ml})$ & $1.4+0.4$ & $1.2+1.1$ & $1.0+0.5$ & $1.5+0.6$ & $1.4+1.0$ \\
\hline$[\text { Triglycerides }]_{\text {plasma }}(\mathrm{mg} / \mathrm{dl})$ & $76+9$ & $81 \pm 13$ & $81 \pm 12$ & $88+11$ & $91 \pm 15$ \\
\hline$[\text { Cholesterol] }]_{\text {plasma }}(\mathrm{mg} / \mathrm{dl})$ & $67 \pm 8$ & $69+8$ & $71 \pm 11$ & $74+8$ & $84+11$ \\
\hline \multicolumn{6}{|c|}{ Obese Zucker Rats } \\
\hline & 6 Weeks & 9 Weeks & 12 Weeks & 15-17 Weeks & 20 Weeks \\
\hline Mass (g) & $184+10^{*}$ & $288 \pm 15^{*}$ & $401 \pm 14^{*}$ & $602 \pm 15^{*}$ & $680 \pm 16^{*}$ \\
\hline Mean Arterial Pressure $(\mathrm{mmHg})$ & $88+5$ & $110+5$ & $118+4^{*}$ & $128+6^{*}$ & $129+5^{*}$ \\
\hline$[\text { Glucose }]_{\text {blood }}(\mathrm{mg} / \mathrm{dl})$ & $102 \pm 12$ & $130+18$ & $168 \pm 16^{*}$ & $184 \pm 18 *$ & $188 \pm 14^{*}$ \\
\hline$[\text { Insulin }]_{\text {plasma }}(\mathrm{ng} / \mathrm{ml})$ & $2.5+0.4^{*}$ & $6.9 \pm 1.1^{*}$ & $8.6 \pm 2.0^{*}$ & $9.4 \pm 1.4^{*}$ & $10.4 \pm 1.9^{*}$ \\
\hline$[\text { Triglycerides }]_{\text {plasma }}(\mathrm{mg} / \mathrm{dl})$ & $122+16^{*}$ & $168 \pm 14^{*}$ & $167 \pm 15^{*}$ & $309 \pm 15^{*}$ & $384 \pm 16^{*}$ \\
\hline$[\text { Cholesterol }]_{\text {plasma }}(\mathrm{mg} / \mathrm{dl})$ & $74+10$ & $85+10$ & $107 \pm 13$ & $128 \pm 12^{*}$ & $136 \pm 12^{*}$ \\
\hline
\end{tabular}




\section{FIGURE LEGENDS}

Figure 1. Microvessel density in the gastrocnemius muscle of 17 week old (Panel A) or temporally progressing (Panel B) lean and obese Zucker rats. Data are presented as mean \pm SEM .

Figure 2. Stimulated vascular NO bioavailability (Panel A) or stimulated vascular thromboxane $B_{2}$ production (Panel $B$ ) measured with amperometric sensors or via ELISA, respectively, as assayed in pooled conduit arteries. Data are presented as mean \pm SEM

Figure 3. Panel A shows microvessel density in the gastrocnemius muscles of lean and obese Zucker rats receiving SQ-29548 therapies or left as untreated controls from 7-13 weeks of age. Panel B demonstrates gastrocnemius muscle microvessel density in animals receiving the same treatment as in Panel $A$, with discontinuation of therapy at 13 weeks but measurement of density continuing to 20 weeks.

Figure 4. Figure 4 displays the elevations in oxidant stress as indicated by plasma nitrotyrosine levels (Panel A) along with effects of anti-ROS therapies on stimulated thromboxane production (Panel C) and ultimate consequence on microvessel density (Panel E). Elevations in TNF $\alpha$ levels are highlighted in Panel B with the effects of antiTNF $\alpha$ therapies on $\mathrm{TxB}_{2}$ (Panel D) levels and microvessel density (Panel F) shown in subsequent panels. 
Figure 1
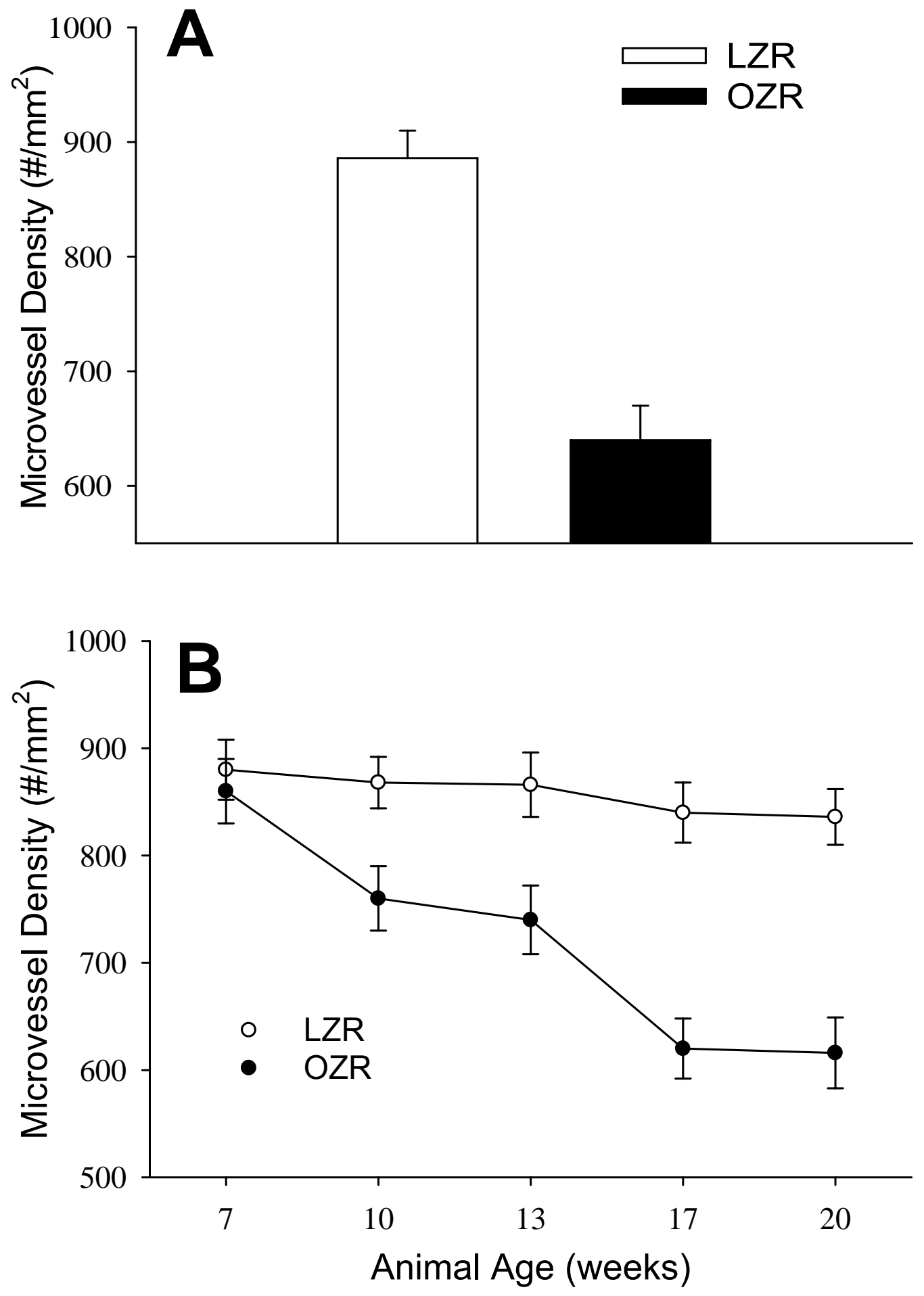
Figure 2
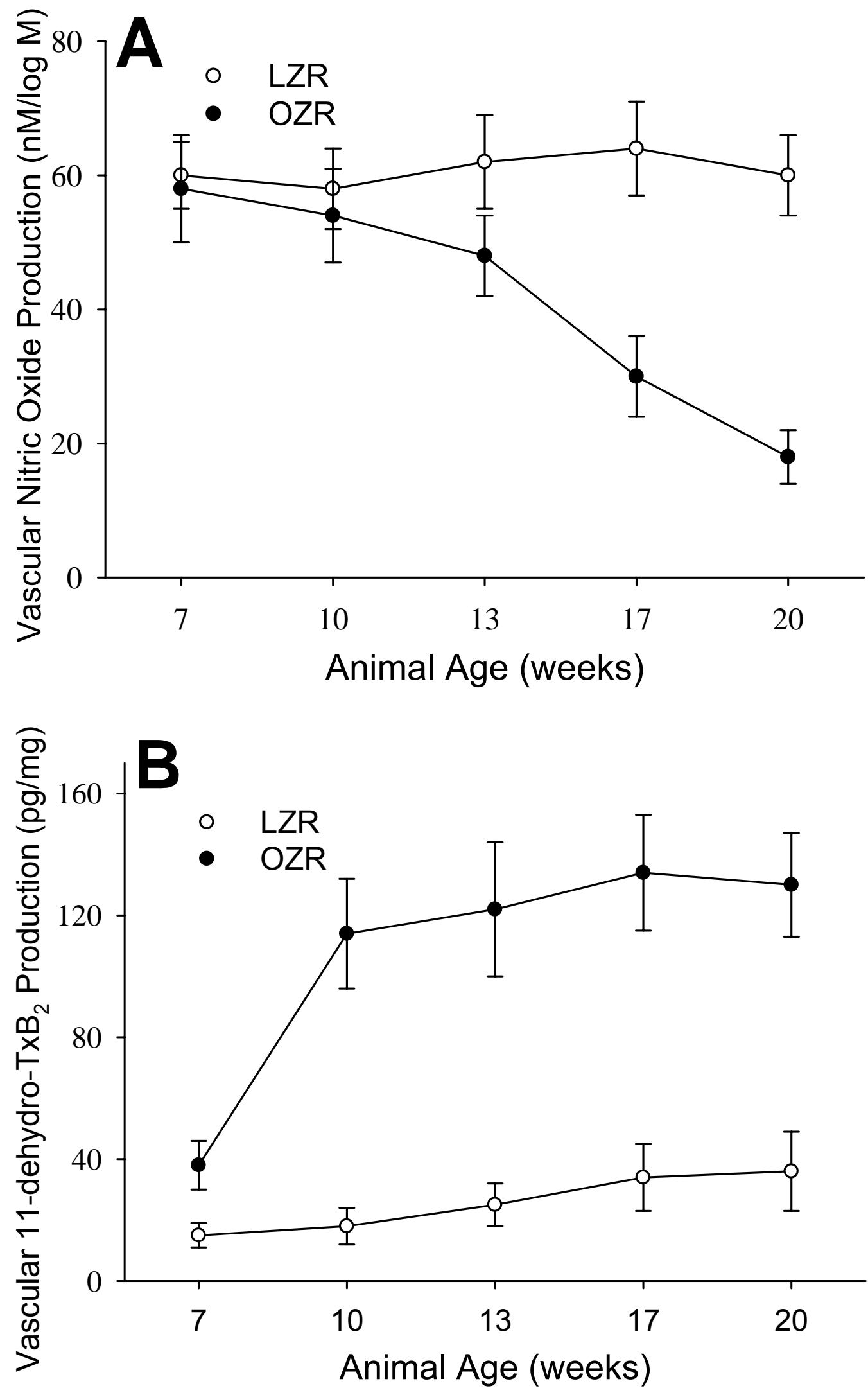
Figure 3
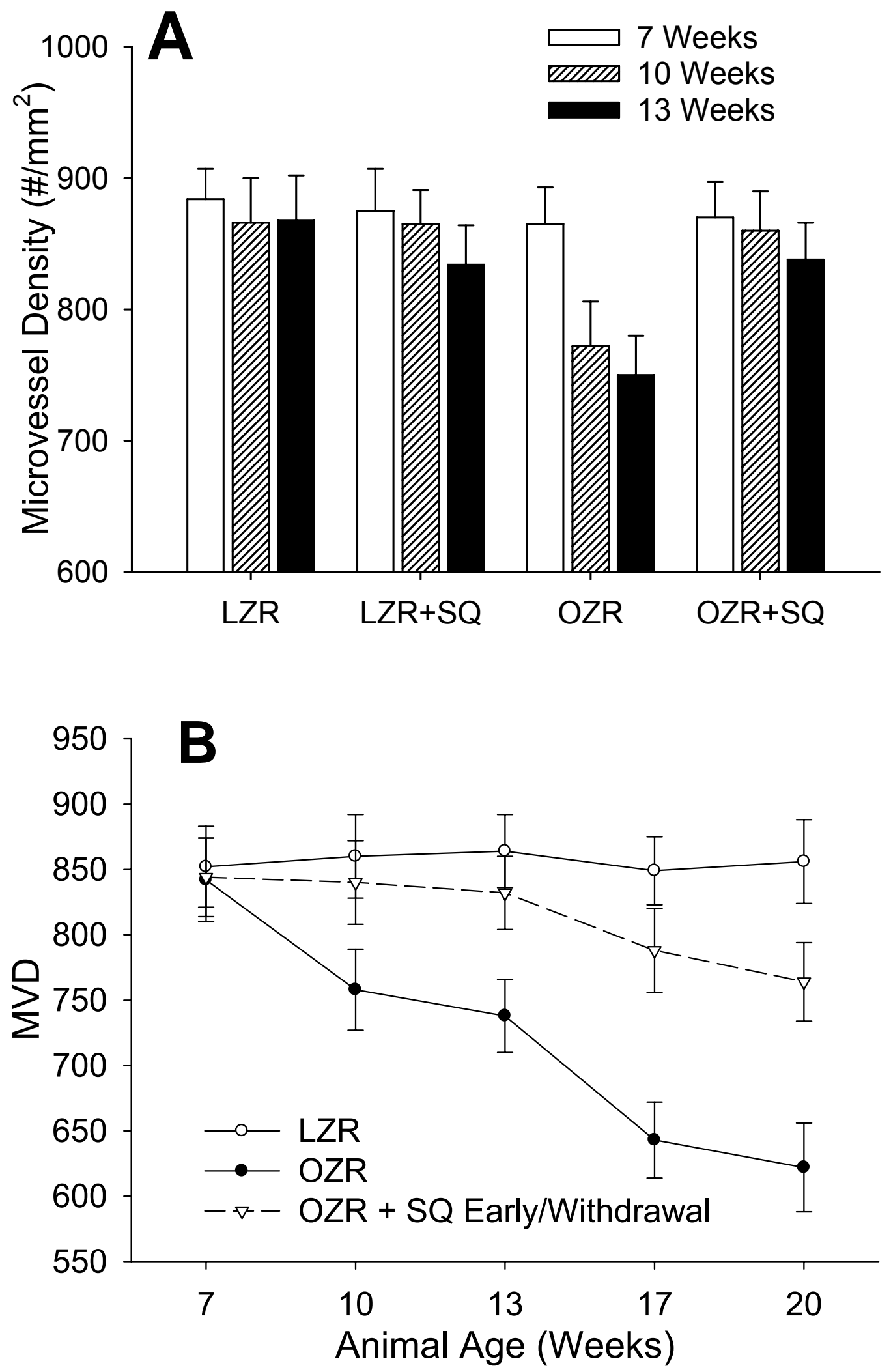
Figure 4
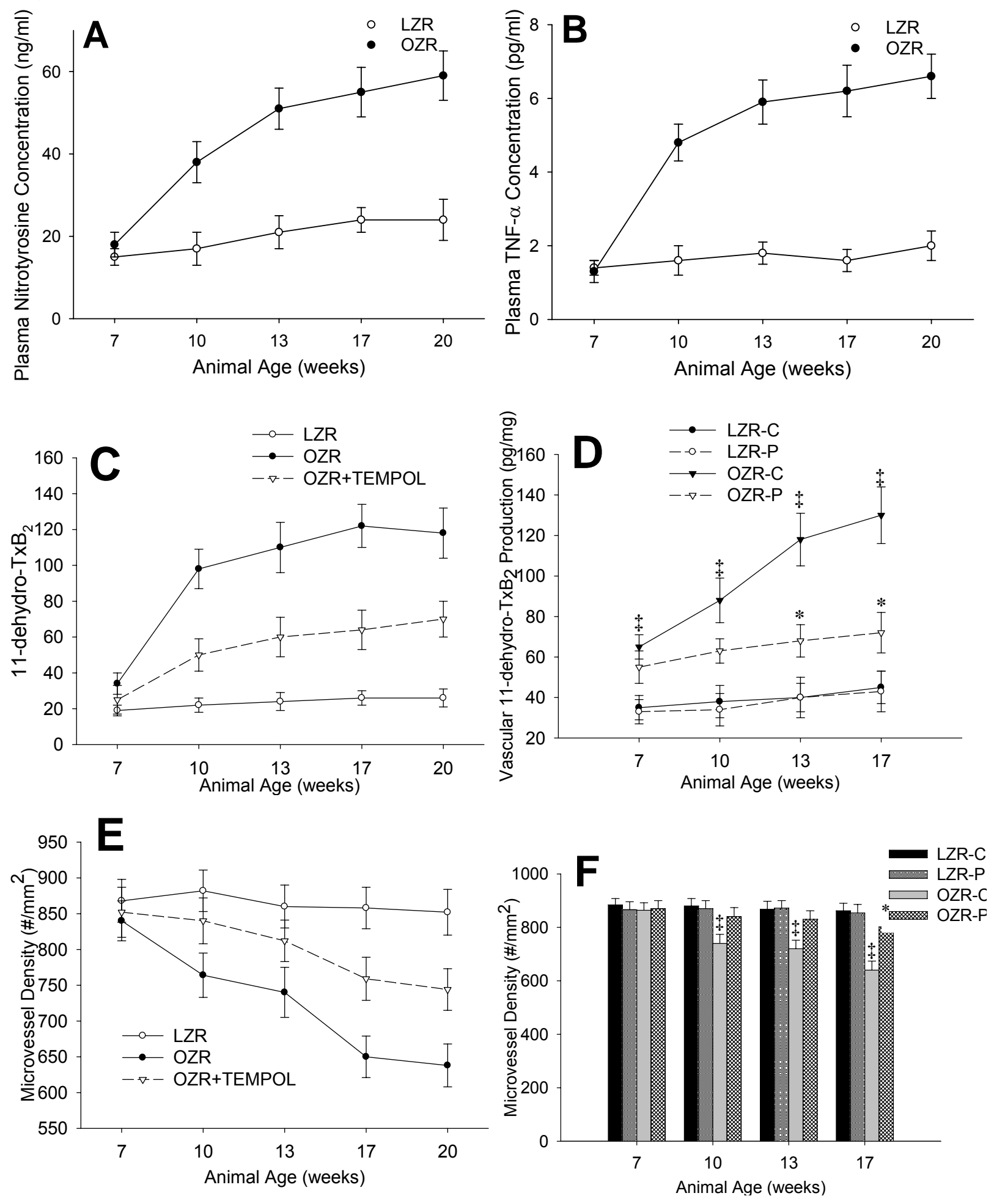
Chapter 6

\section{Discussion}


The purpose of the studies presented within this work was to demonstrate the global systemic consequences of alterations to AA metabolism on both the vasomotor function and vascular network structure within the skeletal muscle of a well-established rodent model of the metabolic syndrome. We first sought to demonstrate that the alterations to AA metabolism, specifically through the cyclooxygenase pathway, do occur and that these alterations have significant consequences on the regulation of skeletal muscle vascular tone. This was accomplished by employing a clean stimulus for the production of prostacyclin, a COX pathway end-product, in both obese Zucker rats and their age matched lean counterparts. This study, which is presented in chapter 2 of this document, was able to demonstrate a blunted hypoxic dilation which was mediated exclusively by modest reductions in prostacyclin production and impressive increases in the production of the vasoconstrictor thromboxane A2 within the vascular endothelium. Further evidence within the same study was able to identify elevations in vascular superoxide levels as a causative factor in the shift from a predominant prostacyclin production induced vasodilation to a blunted vasodilation stemming from pathophysiologic overproduction of thromboxane A2. This phenomenon could be blunted through treatment with a pegylated form of superoxide dismutase which returned production of thromboxane $\mathrm{A} 2$ to a more physiologic level while having no effects on prostacyclin production. Interestingly, while the effects of enhanced superoxide levels on prostacyclin production were non-existent, PEG-SOD treatment was sufficient to largely restore a documented diminished sensitivity of the vessel to prostacyclin to normal levels. Taken together, these data provided the foundational work for investigations into metabolic syndrome related alterations in COX mediated AA 
metabolism as well as the underlying role of superoxide in this imbalance between prostacyclin and thromboxane.

Building on this previous work, investigations were performed in murine models of dyslipidemia, the LDLR knockout mouse and the ApoE knockout mouse. These animals manifest only a single comorbidity of the metabolic syndrome but were interesting as work presented in "Differential Impact of Familial Hypercholesterolemia and Combined Hyperlipidemia on Vascular Wall and Network Remodeling in Mice" by Stapleton et al. provided correlative data between alterations in vascular wall mechanical responses and elevations in thromboxane A2 in these particular animal models. This provided us with the idea that perhaps the dyslipidemia demonstrated in metabolic syndrome, in some way led to the elevations in $\mathrm{TxA}_{2}$ and in turn the vasomotor consequences that had been demonstrated in fully vested animal models of the metabolic syndrome, as detailed in Chapter 2. This provided the impetus for the investigations detailed in Chapter 3, wherein similar alterations to AA metabolism as those found in the Zucker rat are demonstrated with a recapitulation of the significance of enhanced ROS levels as a mediator of these alterations. Further, this manuscript demonstrated the relative insignificance of lipoxygenase and cytochrome p450 pathways in the observed vasculopathies as treatments with inhibitors of each pathway showed no effects on system responses.

Based on this compelling evidence in dyslipidemic mice, and in accordance with documented vasoprotective effects of chronic statin therapies in humans and animal models, we next set out to determine whether chronic reductions in circulating cholesterol levels would be able to restore normal metabolism of AA via COX and, in 
turn, confer a protective effect on the vasculature with regards to vasomotor function. This body of work is summarized in Chapter 4 and clearly demonstrates the ability of statin therapies to protect against metabolic syndrome related vasculopathies regardless of whether those vasculopathies were vasomotor, wall mechanical or network structural in nature. The most significant result of this study was the demonstration that this protection occurs independent of any effects of the therapeutic regimen on cholesterol levels. This was conveyed by treating animals with either statins (HMG-CoA reductase inhibitors) thereby diminishing the biosynthesis of cholesterol or fibrates whose effects increase the fractional clearance of LDL while also diminishing absorption of LDL by the LDL-receptor. Table 1 within chapter 4 illustrates the ability of all therapeutics to significantly lower plasma cholesterol levels in treated animals. However, both the fibrates gemfibrozil and probucol failed to significantly protect against any of the previously mentioned categories of vasculopathy as evidenced by negligible impact of either of these therapies in any of the subsequent figures which assayed acetylcholine mediated dilator responses, gastrocnemius muscle microvessel density and arteriolar incremental distensibility. The key finding of this study was that only those therapeutics that were capable of protecting against a loss of vascular NO bioavailability were capable of conferring vasoprotective effects and these effects in turn, appeared to be mediated by the drugs ability to attenuate chronic inflammatory responses.

We next undertook a more thorough examination of the process of microvascular rarefaction in the metabolic syndrome. The details of Chapter 4 , along with previous work, strongly indicated that microvascular rarefaction is mediated by the loss of 
vascular NO bioavailability and that those therapies capable of protecting against the loss of NO bioavailability had the predictable outcome of protecting against skeletal muscle microvascular rarefaction. It is in the experiments detailed in Chapter 5, where we first set out to determine the time-course of microvascular rarefaction. The initial, unexpected result of this study is shown in Figure 1B of Chapter 5 which clearly demonstrates a biphasic rarefactive process ongoing in these animals. The initial hypothesis upon which this work was operating was that, microvascular rarefaction is a linear process beginning in pre-metabolic syndrome animals with normal microvessel density and progressing in a linear fashion through the time-course of the disease until such time as minimum viable tissue capillarity is achieved. The identification of a biphasic nature to the rarefaction immediately precluded this hypothesis from succeeding to be proven and additionally provided cause to re-evaluate the hypothesis that microvascular rarefaction is mediated by loss of vascular NO bioavailability. It is plausible that if a process demonstrates two temporally distinct phases, those phases may well be mechanistically independent as well.

Investigations into the mechanistic underpinnings of early rarefaction, immediately ruled out reductions in NO bioavailability as a mediator of microvessel loss, as stimulated vascular production of NO is indistinguishable during the time period of early rarefaction when comparing age matched lean and obese Zucker rats. However, late phase microvascular rarefaction demonstrated a strong and inverse relationship to vascular NO bioavailability which, when taken with the previous data, still indicates that the later phase of rarefaction is mediated by NO loss. Based on the previously established information of Chapters 2-4, there was sufficient evidence to indicate the 
possibility that altered inflammatory status and elevations in systemic oxidant stress could be impacting COX mediated metabolism and that this alteration could have effects on rarefaction. The data outlined in Chapter 5 demonstrate that there are alterations to AA metabolism that occur early in the development of the OZR, resulting in drastic elevations in TxA2 during the period of early microvascular rarefaction. Further, when the effects of these elevations are blocked by antagonism of the TxA2 cognate receptor TP by treatment with the competitive antagonist, SQ-29548, there is a complete abolition of early microvascular rarefaction. This effect on early rarefaction can be blunted through alternative pathways, as demonstrated by treatment with the anti-TNFa synthesis compound pentoxifylline or treatment with the oxidant scavenger TEMPOL. Both therapeutics were capable of drastically diminishing elevations in TxA2 and, by extension, early rarefaction. Finally, this study demonstrated the effects of early withdrawl from the compound wherein animals were treated through the entire phase of early rarefaction with the TP receptor antagonist SQ-29548 but then removed from treatment in a fashion temporally consistent with the end of early rarefaction. What was demonstrated was that although rarefaction began anew, this delayed rarefactive process was diminished in intensity from the anticipated 15\% rarefaction that generally occurs with late phase rarefaction to approximately a $6 \%$ loss of total microvessel density.

What requires the greatest scrutiny for future investigation is what mediated that pulse of late rarefaction during the early withdrawl study. Whether this late phase rarefaction was still a NO mediated process or whether it was just a delayed TxA2 mediated rarefaction has yet to be determined. Additionally, regardless of the 
mechanism by which the delayed rarefaction occurred, there was a significant blunting of microvessel loss. This speaks to interplay between early and late rarefaction. It's possible that the early loss of microvessels through the thromboxane mediated process in some way primes the system for the later loss. Alternatively, it's additionally possible that TXA2 elevations in some way sensitize the system to a greater need of NO for maintenance of microvessel density, essentially altering the gain of the system with regards to NO sensitivity and microvessel sparing. Regardless of the mechanism, interplay is apparent as there are obvious effects of blocking early rarefaction on subsequent later microvessel loss.

The supplementary document mentioned in the introduction of this dissertation, "Aspirin Resistance with Genetic Dyslipidemia: Contribution of Vascular Thromboxane Generation" by Frisbee et al. serves as a cautionary to target selection. Aspirin therapy is one of the most commonly prescribed prophylaxes in the world today (5). Numerous studies have demonstrated the ability to increase the likelihood of survivability of major cardiac events in those individuals on a chronic, low-dose, aspirin regimen (2-6). Aspirin serves in this capacity through its ability as a COX-2 inhibitor, decreasing TxA2 production by platelets and diminishing platelet aggregation with the result of reducing chance of thrombus formation (2). Based on this work, COX-2 selective drugs have been developed for the purpose of prophylaxis primarily in individuals with some form of chronic inflammatory disease. What has received significant media attention is the voluntary recall of the selective COX-2 inhibitor Vioxx which was found to significantly increase the probability of negative outcomes from a major cardiovascular event. To many, this finding was unsurprising since you lose the contributions of prostacyclin, 
prostaglandins and thromboxane in the regulation of vascular tone through this highly upstream inhibition. Where Appendix II serves as a cautionary is in its demonstration of dyslipidemic mice to lose sensitivity to chronic aspirin therapies. Essentially, after any significant period of time with profound dyslipidemia, in mice, the prophylaxis with Aspirin loses any therapeutic value. While studies have to be performed in a fully vested model of metabolic syndrome, logical extension is that since these animals demonstrate similarly profound dyslipidemia, a similar resistant phenotype will emerge. Additionally, since the vasomotor and network consequences of COX metabolites seems to stem from an imbalance in their production as opposed to a uniform overproduction of all COX metabolites, more selective downstream targets will logically have greater therapeutic potential.

Conjecture based on the data presented within this work leads to the following conclusions. There are significant consequences to the peripheral vasculature resulting from an imbalance of COX metabolites with moderately diminished prostacyclin production and sensitivity coupled with drastically elevated TxA2 production resulting in enhanced vascular tone, blunted dilator responses, increased vessel wall stiffness and profound microvascular rarefaction. Evidence from clinical trials indicates that the adverse effects of chronic COX inhibition severely outweigh therapeutic potential. Additionally, animal models of dyslipidemia whose lipid levels mimic those found in metabolic syndrome models demonstrate the very real potential of chronic COX therapies being ineffectual with time as a resistant phenotype emerges. Metabolic syndrome individuals require a better therapeutic target for therapies to disrupt these cOX mediated effects. In the short term, the answers would seem to come from 
antagonism of the TxA2 dependent pathways which have been shown to protect against rarefaction and blunted dilator responses in animal models of metabolic syndrome.

While the above mentioned therapies show promise for the short-term, where they fail is in a mechanistic understanding of the cause of alterations in COX metabolite balance. Inhibition of the TxA2 pathway still does improve the production of prostacyclin. While a more physiologic ratio of TxA2 to prostacyclin may be achievable simply through TxA2 pathway antagonism, this doesn't correct the diminished sensitivity of the system to prostacyclin. Chronic anti-oxidant therapies demonstrate the ability to confer partial protection against rarefaction along with partial restoration of normal vasomotor responses while also returning sensitivity of the system to prostacyclin. This combined with similar effects of anti-inflammatory drugs would indicate that while the processes of vascular dysfunction are resulting from alterations to COX metabolite balances, the origin of the problem is not in AA metabolism but rather the effects of chronic inflammation and elevations in oxidant stress on the system. Further studies are required to determine the origins of this inflammation and oxidant stress to begin to truly treat the root of a problem that appears to extend significantly beyond AA metabolism.

Additionally, future studies need to redouble their efforts on the understanding of metabolic syndrome related skeletal muscle microvascular rarefaction. Work by Frisbee et al. has demonstrated that in the obese Zucker rat, when allowed to reach fully established metabolic syndrome symptomology, even a fully dilated vascular bed is incapable of matching perfusion with increased metabolic demand resulting in more rapid rates of fatigue and reduced maximal muscle tension development (1). This 
inability to match metabolic demand with perfusion is a hallmark of metabolic syndrome and is too readily attributed to the complex interplay of vasomotor regulators. While there is no doubt that the dysregulation of the vasomotor plays a significant role in demand:perfusion impairment, the ability to adequately repair a complex system such as regulation of vascular tone will require extensive further studies. On the contrary, making sure there is adequate tissue capillarity to maintain bulk flow though may be a much more correctable problem. Rarefaction itself has largely been ignored owing to the fact that tissues work with a surplus capillarity and even when working with a deficit, the ability to regulate tone allows a tissue to maintain adequate perfusion much more readily than if vessels were passive. This fails to take into account that with each capillary lost, the error term for proper vasomotor regulation must become proportionately smaller to maintain normal flow. The caveat being that if tissue capillarity can be spared, this error term can afford to remain much larger. Taken together these data suggest that further investigations into understanding the underlying mechanisms resulting in elevations in oxidant stress and inflammatory status are essential to understanding the true cause of obesity related vascular disease and that it is only through an understanding of the origins of these problems that a true therapeutic regimen against these multi-faceted vasculopathies can be developed. 


\section{Reference List}

1. Frisbee JC, Goodwill AG, Butcher JT and Olfert IM. Divergence between arterial perfusion and fatigue resistance in skeletal muscle in the metabolic syndrome. Exp Physiol 96: 369-383, 2011.

2. Giannarelli C, Zafar MU and Badimon JJ. Prostanoid and TP-receptors in atherothrombosis: is there a role for their antagonism? Thromb Haemost 104: 949954, 2010.

3. Hsiao FY, Tsai YW, Huang WF, Wen YW, Chen PF, Chang PY and Kuo KN. A comparison of aspirin and clopidogrel with or without proton pump inhibitors for the secondary prevention of cardiovascular events in patients at high risk for gastrointestinal bleeding. Clin Ther 31: 2038-2047, 2009.

4. Ong G, Davis TM and Davis WA. Aspirin is associated with reduced cardiovascular and all-cause mortality in type 2 diabetes in a primary prevention setting: the Fremantle Diabetes study. Diabetes Care 33: 317-321, 2010.

5. Pignone $\mathbf{M}$ and Williams $\mathbf{C D}$. Aspirin for primary prevention of cardiovascular disease in diabetes mellitus. Nat Rev Endocrinol 6: 619-628, 2010. 
6. Rafferty M, Walters MR and Dawson J. Anti-platelet therapy and aspirin resistance - clinically and chemically relevant? Curr Med Chem 17: 4578-4586, 2010. 


\section{Permission to Reproduce Copyrighted Materials}




\section{Copyright} Clearance Center

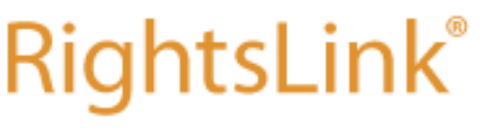

Title:

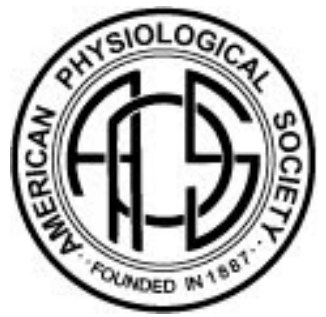

Increased vascular thromboxane generation impairs dilation of skeletal muscle arterioles of obese Zucker rats with reduced oxygen tension

Author: Adam G. Goodwill, Milinda E. James, J efferson C. Frisbee

Publication: Am J Physiol- Heart and Circulatory Physiology

Publisher: The American Physiological Society

Date: Oct 1, 2008

Copyright @ 2008, The American Physiological Society
Logged in as:

Adam Goodwill

LOGOUT

\section{Permission Not Required}

Permission is not required for this type of use.

\section{BACK}

\section{CLOSE WINDOW}

Copyright (c) 2011 Copyright Clearance Center, Inc. All Rights Reserved. Privacy statement. Comments? We would like to hear from you. E-mail us at customercare@copyright.com 


\section{JOHN WILEY AND SONS LICENSE TERMS AND CONDITIONS}

Nov 20, 2011

This is a License Agreement between Adam G Goodwill ("You") and John Wiley and Sons ("John Wiley and Sons") provided by Copyright Clearance Center ("CCC"). The license consists of your order details, the terms and conditions provided by John Wiley and Sons, and the payment terms and conditions.

\section{All payments must be made in full to CCC. For payment instructions, please see information listed at the bottom of this form.}

License Number

License date

Licensed content publisher

Licensed content publication

Licensed content title

Licensed content author

Licensed content date

Start page

End page

Type of use

Requestor type

Format

Portion

Will you be translating?

Order reference number

Total

0.00 USD

621

631

No

\section{3}

Nov 20, 2011

John Wiley and Sons

Microcirculation

Increased Arachidonic Acid-Induced Thromboxane Generation Impairs Skeletal Muscle Arteriolar Dilation with Genetic Dyslipidemia ADAM. G. GOODWILL,PHOEBE A. STAPLETON,MILINDA E. JAMES,ALEXANDRE C. D'AUDIFFRET,JEFFERSON C. FRISBEE

Oct 1, 2008

Dissertation/Thesis

Author of this Wiley article

Print and electronic

Full article

Terms and Conditions

\section{TERMS AND CONDITIONS}

This copyrighted material is owned by or exclusively licensed to John Wiley \& Sons, Inc. or one of its group companies (each a "Wiley Company") or a society for whom a Wiley Company has exclusive publishing rights in relation to a particular journal (collectively WILEY"). By clicking "accept" in connection with completing this licensing transaction, you agree that the following terms and conditions apply to this transaction (along with the billing and payment terms and conditions established by the Copyright Clearance Center Inc., ("CCC's Billing and Payment terms and conditions"), at the time that you opened your Rightslink account (these are available at any time at http://myaccount.copyright.com)

Terms and Conditions

1. The materials you have requested permission to reproduce (the "Materials") are protected by copyright. 
2. You are hereby granted a personal, non-exclusive, non-sublicensable, non-transferable, worldwide, limited license to reproduce the Materials for the purpose specified in the licensing process. This license is for a one-time use only with a maximum distribution equal to the number that you identified in the licensing process. Any form of republication granted by this licence must be completed within two years of the date of the grant of this licence (although copies prepared before may be distributed thereafter). The Materials shall not be used in any other manner or for any other purpose. Permission is granted subject to an appropriate acknowledgement given to the author, title of the material/book/journal and the publisher. You shall also duplicate the copyright notice that appears in the Wiley publication in your use of the Material. Permission is also granted on the understanding that nowhere in the text is a previously published source acknowledged for all or part of this Material. Any third party material is expressly excluded from this permission.

3. With respect to the Materials, all rights are reserved. Except as expressly granted by the terms of the license, no part of the Materials may be copied, modified, adapted (except for minor reformatting required by the new Publication), translated, reproduced, transferred or distributed, in any form or by any means, and no derivative works may be made based on the Materials without the prior permission of the respective copyright owner. You may not alter, remove or suppress in any manner any copyright, trademark or other notices displayed by the Materials. You may not license, rent, sell, loan, lease, pledge, offer as security, transfer or assign the Materials, or any of the rights granted to you hereunder to any other person.

4. The Materials and all of the intellectual property rights therein shall at all times remain the exclusive property of John Wiley \& Sons Inc or one of its related companies (WILEY) or their respective licensors, and your interest therein is only that of having possession of and the right to reproduce the Materials pursuant to Section 2 herein during the continuance of this Agreement. You agree that you own no right, title or interest in or to the Materials or any of the intellectual property rights therein. You shall have no rights hereunder other than the license as provided for above in Section 2. No right, license or interest to any trademark, trade name, service mark or other branding ("Marks") of WILEY or its licensors is granted hereunder, and you agree that you shall not assert any such right, license or interest with respect thereto.

5. NEITHER WILEY NOR ITS LICENSORS MAKES ANY WARRANTY OR REPRESENTATION OF ANY KIND TO YOU OR ANY THIRD PARTY, EXPRESS, IMPLIED OR STATUTORY, WITH RESPECT TO THE MATERIALS OR THE ACCURACY OF ANY INFORMATION CONTAINED IN THE MATERIALS, INCLUDING, WITHOUT LIMITATION, ANY IMPLIED WARRANTY OF MERCHANTABILITY, ACCURACY, SATISFACTORY QUALITY, FITNESS FOR A PARTICULAR PURPOSE, USABILITY, INTEGRATION OR NON-INFRINGEMENT AND ALL SUCH WARRANTIES ARE HEREBY EXCLUDED BY WILEY AND ITS LICENSORS AND WAIVED BY YOU.

6. WILEY shall have the right to terminate this Agreement immediately upon breach of this Agreement by you.

7. You shall indemnify, defend and hold harmless WILEY, its Licensors and their respective directors, officers, agents and employees, from and against any actual or threatened claims, demands, causes of action or proceedings arising from any breach of this Agreement by you.

8. IN NO EVENT SHALL WILEY OR ITS LICENSORS BE LIABLE TO YOU OR ANY OTHER PARTY OR ANY OTHER PERSON OR ENTITY FOR ANY SPECIAL, CONSEQUENTIAL, INCIDENTAL, INDIRECT, EXEMPLARY OR PUNITIVE DAMAGES, HOWEVER CAUSED, ARISING OUT OF OR IN CONNECTION WITH THE DOWNLOADING, PROVISIONING, VIEWING OR USE OF THE MATERIALS REGARDLESS OF THE FORM OF ACTION, WHETHER FOR BREACH OF CONTRACT, BREACH OF WARRANTY, TORT, NEGLIGENCE, INFRINGEMENT OR OTHERWISE (INCLUDING, WITHOUT LIMITATION, DAMAGES BASED ON LOSS OF PROFITS, DATA, FILES, USE, BUSINESS OPPORTUNITY OR CLAIMS OF THIRD PARTIES), AND WHETHER OR NOT THE PARTY HAS BEEN ADVISED OF THE POSSIBILITY OF SUCH DAMAGES. THIS LIMITATION SHALL APPLY NOTWITHSTANDING ANY FAILURE OF ESSENTIAL PURPOSE OF ANY LIMITED REMEDY PROVIDED HEREIN.

9. Should any provision of this Agreement be held by a court of competent jurisdiction to be illegal, invalid, or unenforceable, that provision shall be deemed amended to achieve as nearly as possible the same economic effect as the original provision, and the legality, validity and 
enforceability of the remaining provisions of this Agreement shall not be affected or impaired thereby.

10. The failure of either party to enforce any term or condition of this Agreement shall not constitute a waiver of either party's right to enforce each and every term and condition of this Agreement. No breach under this agreement shall be deemed waived or excused by either party unless such waiver or consent is in writing signed by the party granting such waiver or consent. The waiver by or consent of a party to a breach of any provision of this Agreement shall not operate or be construed as a waiver of or consent to any other or subsequent breach by such other party.

11. This Agreement may not be assigned (including by operation of law or otherwise) by you without WILEY's prior written consent.

12. Any fee required for this permission shall be non-refundable after thirty (30) days from receipt.

13. These terms and conditions together with CCC's Billing and Payment terms and conditions (which are incorporated herein) form the entire agreement between you and WILEY concerning this licensing transaction and (in the absence of fraud) supersedes all prior agreements and representations of the parties, oral or written. This Agreement may not be amended except in writing signed by both parties. This Agreement shall be binding upon and inure to the benefit of the parties' successors, legal representatives, and authorized assigns.

14. In the event of any conflict between your obligations established by these terms and conditions and those established by CCC's Billing and Payment terms and conditions, these terms and conditions shall prevail.

15. WILEY expressly reserves all rights not specifically granted in the combination of (i) the license details provided by you and accepted in the course of this licensing transaction, (ii) these terms and conditions and (iii) CCC's Billing and Payment terms and conditions.

16. This Agreement will be void if the Type of Use, Format, Circulation, or Requestor Type was misrepresented during the licensing process.

17. This Agreement shall be governed by and construed in accordance with the laws of the State of New York, USA, without regards to such state's conflict of law rules. Any legal action, suit or proceeding arising out of or relating to these Terms and Conditions or the breach thereof shall be instituted in a court of competent jurisdiction in New York County in the State of New York in the United States of America and each party hereby consents and submits to the personal jurisdiction of such court, waives any objection to venue in such court and consents to service of process by registered or certified mail, return receipt requested, at the last known address of such party.

\section{Wiley Open Access Terms and Conditions}

All research articles published in Wiley Open Access journals are fully open access: immediately freely available to read, download and share. Articles are published under the terms of the Creative Commons Attribution Non Commercial License. which permits use, distribution and reproduction in any medium, provided the original work is properly cited and is not used for commercial purposes. The license is subject to the Wiley Open Access terms and conditions: Wiley Open Access articles are protected by copyright and are posted to repositories and websites in accordance with the terms of the Creative Commons Attribution Non Commercial License. At the time of deposit, Wiley Open Access articles include all changes made during peer review, copyediting, and publishing. Repositories and websites that host the article are responsible for incorporating any publisher-supplied amendments or retractions issued subsequently. Wiley Open Access articles are also available without charge on Wiley's publishing platform, Wiley Online Library or any successor sites.

\section{Use by non-commercial users}

For non-commercial and non-promotional purposes individual users may access, download, copy, 
display and redistribute to colleagues Wiley Open Access articles, as well as adapt, translate, textand data-mine the content subject to the following conditions:

- The authors' moral rights are not compromised. These rights include the right of "paternity" (also known as "attribution" - the right for the author to be identified as such) and "integrity" (the right for the author not to have the work altered in such a way that the author's reputation or integrity may be impugned).

- Where content in the article is identified as belonging to a third party, it is the obligation of the user to ensure that any reuse complies with the copyright policies of the owner of that content.

- If article content is copied, downloaded or otherwise reused for non-commercial research and education purposes, a link to the appropriate bibliographic citation (authors, journal, article title, volume, issue, page numbers, DOI and the link to the definitive published version on Wiley Online Library) should be maintained. Copyright notices and disclaimers must not be deleted.

- Any translations, for which a prior translation agreement with Wiley has not been agreed, must prominently display the statement: "This is an unofficial translation of an article that appeared in a Wiley publication. The publisher has not endorsed this translation."

Use by commercial "for-profit" organisations

Use of Wiley Open Access articles for commercial, promotional, or marketing purposes requires further explicit permission from Wiley and will be subject to a fee. Commercial purposes include:

- Copying or downloading of articles, or linking to such articles for further redistribution, sale or licensing;

- Copying, downloading or posting by a site or service that incorporates advertising with such content;

- The inclusion or incorporation of article content in other works or services (other than normal quotations with an appropriate citation) that is then available for sale or licensing, for a fee (for example, a compilation produced for marketing purposes, inclusion in a sales pack)

- Use of article content (other than normal quotations with appropriate citation) by forprofit organisations for promotional purposes

- Linking to article content in e-mails redistributed for promotional, marketing or educational purposes;

- Use for the purposes of monetary reward by means of sale, resale, licence, loan, transfer or other form of commercial exploitation such as marketing products

- Print reprints of Wiley Open Access articles can be purchased from: corporatesales@,wiley.com

Other Terms and Conditions:

BY CLICKING ON THE "I AGREE..." BOX, YOU ACKNOWLEDGE THAT YOU HAVE READ AND FULLY UNDERSTAND EACH OF THE SECTIONS OF AND PROVISIONS SET FORTH IN THIS AGREEMENT AND THAT YOU ARE IN AGREEMENT WITH AND ARE WILLING TO ACCEPT ALL OF YOUR OBLIGATIONS AS SET FORTH IN THIS AGREEMENT.

v1.7

If you would like to pay for this license now, please remit this license along with your 
payment made payable to "COPYRIGHT CLEARANCE CENTER" otherwise you will be invoiced within 48 hours of the license date. Payment should be in the form of a check or money order referencing your account number and this invoice number

RLNK500668320.

Once you receive your invoice for this order, you may pay your invoice by credit card. Please follow instructions provided at that time.

Make Payment To:

Copyright Clearance Center

Dept 001

P.O. Box 843006

Boston, MA 02284-3006

For suggestions or comments regarding this order, contact RightsLink Customer Support: customercare@copyright.com or +1-877-622-5543 (toll free in the US) or +1978-646-2777.

Gratis licenses (referencing $\mathbf{\$ 0}$ in the Total field) are free. Please retain this printable license for your reference. No payment is required. 


\section{JOHN WILEY AND SONS LICENSE TERMS AND CONDITIONS}

Nov 20, 2011

This is a License Agreement between Adam G Goodwill ("You") and John Wiley and Sons ("John Wiley and Sons") provided by Copyright Clearance Center ("CCC"). The license consists of your order details, the terms and conditions provided by John Wiley and Sons, and the payment terms and conditions.

\section{All payments must be made in full to CCC. For payment instructions, please see information listed at the bottom of this form.}

License Number

License date

Licensed content publisher

Licensed content publication

Licensed content title

Licensed content author

Licensed content date

Start page

End page

Type of use

Requestor type

Format

Portion

Will you be translating?

Order reference number

Total

0.00 USD

667

684

No

$$
2793350595703
$$

Nov 20, 2011

John Wiley and Sons

Microcirculation

Impact of Chronic Anticholesterol Therapy on Development of Microvascular Rarefaction in the Metabolic Syndrome

Adam G. Goodwill,Stephanie J. Frisbee,Phoebe A. Stapleton,Milinda E. James, Jefferson C. Frisbee

Nov 1, 2009

Dissertation/Thesis

Author of this Wiley article

Print and electronic

Full article

Terms and Conditions

\section{TERMS AND CONDITIONS}

This copyrighted material is owned by or exclusively licensed to John Wiley \& Sons, Inc. or one of its group companies (each a "Wiley Company") or a society for whom a Wiley Company has exclusive publishing rights in relation to a particular journal (collectively WILEY"). By clicking "accept" in connection with completing this licensing transaction, you agree that the following terms and conditions apply to this transaction (along with the billing and payment terms and conditions established by the Copyright Clearance Center Inc., ("CCC's Billing and Payment terms and conditions"), at the time that you opened your Rightslink account (these are available at any time at http://myaccount.copyright.com)

Terms and Conditions

1. The materials you have requested permission to reproduce (the "Materials") are protected by copyright. 
2. You are hereby granted a personal, non-exclusive, non-sublicensable, non-transferable, worldwide, limited license to reproduce the Materials for the purpose specified in the licensing process. This license is for a one-time use only with a maximum distribution equal to the number that you identified in the licensing process. Any form of republication granted by this licence must be completed within two years of the date of the grant of this licence (although copies prepared before may be distributed thereafter). The Materials shall not be used in any other manner or for any other purpose. Permission is granted subject to an appropriate acknowledgement given to the author, title of the material/book/journal and the publisher. You shall also duplicate the copyright notice that appears in the Wiley publication in your use of the Material. Permission is also granted on the understanding that nowhere in the text is a previously published source acknowledged for all or part of this Material. Any third party material is expressly excluded from this permission.

3. With respect to the Materials, all rights are reserved. Except as expressly granted by the terms of the license, no part of the Materials may be copied, modified, adapted (except for minor reformatting required by the new Publication), translated, reproduced, transferred or distributed, in any form or by any means, and no derivative works may be made based on the Materials without the prior permission of the respective copyright owner. You may not alter, remove or suppress in any manner any copyright, trademark or other notices displayed by the Materials. You may not license, rent, sell, loan, lease, pledge, offer as security, transfer or assign the Materials, or any of the rights granted to you hereunder to any other person.

4. The Materials and all of the intellectual property rights therein shall at all times remain the exclusive property of John Wiley \& Sons Inc or one of its related companies (WILEY) or their respective licensors, and your interest therein is only that of having possession of and the right to reproduce the Materials pursuant to Section 2 herein during the continuance of this Agreement. You agree that you own no right, title or interest in or to the Materials or any of the intellectual property rights therein. You shall have no rights hereunder other than the license as provided for above in Section 2. No right, license or interest to any trademark, trade name, service mark or other branding ("Marks") of WILEY or its licensors is granted hereunder, and you agree that you shall not assert any such right, license or interest with respect thereto.

5. NEITHER WILEY NOR ITS LICENSORS MAKES ANY WARRANTY OR REPRESENTATION OF ANY KIND TO YOU OR ANY THIRD PARTY, EXPRESS, IMPLIED OR STATUTORY, WITH RESPECT TO THE MATERIALS OR THE ACCURACY OF ANY INFORMATION CONTAINED IN THE MATERIALS, INCLUDING, WITHOUT LIMITATION, ANY IMPLIED WARRANTY OF MERCHANTABILITY, ACCURACY, SATISFACTORY QUALITY, FITNESS FOR A PARTICULAR PURPOSE, USABILITY, INTEGRATION OR NON-INFRINGEMENT AND ALL SUCH WARRANTIES ARE HEREBY EXCLUDED BY WILEY AND ITS LICENSORS AND WAIVED BY YOU.

6. WILEY shall have the right to terminate this Agreement immediately upon breach of this Agreement by you.

7. You shall indemnify, defend and hold harmless WILEY, its Licensors and their respective directors, officers, agents and employees, from and against any actual or threatened claims, demands, causes of action or proceedings arising from any breach of this Agreement by you.

8. IN NO EVENT SHALL WILEY OR ITS LICENSORS BE LIABLE TO YOU OR ANY OTHER PARTY OR ANY OTHER PERSON OR ENTITY FOR ANY SPECIAL, CONSEQUENTIAL, INCIDENTAL, INDIRECT, EXEMPLARY OR PUNITIVE DAMAGES, HOWEVER CAUSED, ARISING OUT OF OR IN CONNECTION WITH THE DOWNLOADING, PROVISIONING, VIEWING OR USE OF THE MATERIALS REGARDLESS OF THE FORM OF ACTION, WHETHER FOR BREACH OF CONTRACT, BREACH OF WARRANTY, TORT, NEGLIGENCE, INFRINGEMENT OR OTHERWISE (INCLUDING, WITHOUT LIMITATION, DAMAGES BASED ON LOSS OF PROFITS, DATA, FILES, USE, BUSINESS OPPORTUNITY OR CLAIMS OF THIRD PARTIES), AND WHETHER OR NOT THE PARTY HAS BEEN ADVISED OF THE POSSIBILITY OF SUCH DAMAGES. THIS LIMITATION SHALL APPLY NOTWITHSTANDING ANY FAILURE OF ESSENTIAL PURPOSE OF ANY LIMITED REMEDY PROVIDED HEREIN.

9. Should any provision of this Agreement be held by a court of competent jurisdiction to be illegal, invalid, or unenforceable, that provision shall be deemed amended to achieve as nearly as possible the same economic effect as the original provision, and the legality, validity and 
enforceability of the remaining provisions of this Agreement shall not be affected or impaired thereby.

10. The failure of either party to enforce any term or condition of this Agreement shall not constitute a waiver of either party's right to enforce each and every term and condition of this Agreement. No breach under this agreement shall be deemed waived or excused by either party unless such waiver or consent is in writing signed by the party granting such waiver or consent. The waiver by or consent of a party to a breach of any provision of this Agreement shall not operate or be construed as a waiver of or consent to any other or subsequent breach by such other party.

11. This Agreement may not be assigned (including by operation of law or otherwise) by you without WILEY's prior written consent.

12. Any fee required for this permission shall be non-refundable after thirty (30) days from receipt.

13. These terms and conditions together with CCC's Billing and Payment terms and conditions (which are incorporated herein) form the entire agreement between you and WILEY concerning this licensing transaction and (in the absence of fraud) supersedes all prior agreements and representations of the parties, oral or written. This Agreement may not be amended except in writing signed by both parties. This Agreement shall be binding upon and inure to the benefit of the parties' successors, legal representatives, and authorized assigns.

14. In the event of any conflict between your obligations established by these terms and conditions and those established by CCC's Billing and Payment terms and conditions, these terms and conditions shall prevail.

15. WILEY expressly reserves all rights not specifically granted in the combination of (i) the license details provided by you and accepted in the course of this licensing transaction, (ii) these terms and conditions and (iii) CCC's Billing and Payment terms and conditions.

16. This Agreement will be void if the Type of Use, Format, Circulation, or Requestor Type was misrepresented during the licensing process.

17. This Agreement shall be governed by and construed in accordance with the laws of the State of New York, USA, without regards to such state's conflict of law rules. Any legal action, suit or proceeding arising out of or relating to these Terms and Conditions or the breach thereof shall be instituted in a court of competent jurisdiction in New York County in the State of New York in the United States of America and each party hereby consents and submits to the personal jurisdiction of such court, waives any objection to venue in such court and consents to service of process by registered or certified mail, return receipt requested, at the last known address of such party.

\section{Wiley Open Access Terms and Conditions}

All research articles published in Wiley Open Access journals are fully open access: immediately freely available to read, download and share. Articles are published under the terms of the Creative Commons Attribution Non Commercial License. which permits use, distribution and reproduction in any medium, provided the original work is properly cited and is not used for commercial purposes. The license is subject to the Wiley Open Access terms and conditions: Wiley Open Access articles are protected by copyright and are posted to repositories and websites in accordance with the terms of the Creative Commons Attribution Non Commercial License. At the time of deposit, Wiley Open Access articles include all changes made during peer review, copyediting, and publishing. Repositories and websites that host the article are responsible for incorporating any publisher-supplied amendments or retractions issued subsequently. Wiley Open Access articles are also available without charge on Wiley's publishing platform, Wiley Online Library or any successor sites.

\section{Use by non-commercial users}

For non-commercial and non-promotional purposes individual users may access, download, copy, 
display and redistribute to colleagues Wiley Open Access articles, as well as adapt, translate, textand data-mine the content subject to the following conditions:

- The authors' moral rights are not compromised. These rights include the right of "paternity" (also known as "attribution" - the right for the author to be identified as such) and "integrity" (the right for the author not to have the work altered in such a way that the author's reputation or integrity may be impugned).

- Where content in the article is identified as belonging to a third party, it is the obligation of the user to ensure that any reuse complies with the copyright policies of the owner of that content.

- If article content is copied, downloaded or otherwise reused for non-commercial research and education purposes, a link to the appropriate bibliographic citation (authors, journal, article title, volume, issue, page numbers, DOI and the link to the definitive published version on Wiley Online Library) should be maintained. Copyright notices and disclaimers must not be deleted.

- Any translations, for which a prior translation agreement with Wiley has not been agreed, must prominently display the statement: "This is an unofficial translation of an article that appeared in a Wiley publication. The publisher has not endorsed this translation."

Use by commercial "for-profit" organisations

Use of Wiley Open Access articles for commercial, promotional, or marketing purposes requires further explicit permission from Wiley and will be subject to a fee. Commercial purposes include:

- Copying or downloading of articles, or linking to such articles for further redistribution, sale or licensing;

- Copying, downloading or posting by a site or service that incorporates advertising with such content;

- The inclusion or incorporation of article content in other works or services (other than normal quotations with an appropriate citation) that is then available for sale or licensing, for a fee (for example, a compilation produced for marketing purposes, inclusion in a sales pack)

- Use of article content (other than normal quotations with appropriate citation) by forprofit organisations for promotional purposes

- Linking to article content in e-mails redistributed for promotional, marketing or educational purposes;

- Use for the purposes of monetary reward by means of sale, resale, licence, loan, transfer or other form of commercial exploitation such as marketing products

- Print reprints of Wiley Open Access articles can be purchased from: corporatesales@,wiley.com

Other Terms and Conditions:

BY CLICKING ON THE "I AGREE..." BOX, YOU ACKNOWLEDGE THAT YOU HAVE READ AND FULLY UNDERSTAND EACH OF THE SECTIONS OF AND PROVISIONS SET FORTH IN THIS AGREEMENT AND THAT YOU ARE IN AGREEMENT WITH AND ARE WILLING TO ACCEPT ALL OF YOUR OBLIGATIONS AS SET FORTH IN THIS AGREEMENT.

v1.7

If you would like to pay for this license now, please remit this license along with your 
payment made payable to "COPYRIGHT CLEARANCE CENTER" otherwise you will be invoiced within 48 hours of the license date. Payment should be in the form of a check or money order referencing your account number and this invoice number

RLNK500668325.

Once you receive your invoice for this order, you may pay your invoice by credit card. Please follow instructions provided at that time.

Make Payment To:

Copyright Clearance Center

Dept 001

P.O. Box 843006

Boston, MA 02284-3006

For suggestions or comments regarding this order, contact RightsLink Customer Support: customercare@copyright.com or +1-877-622-5543 (toll free in the US) or +1978-646-2777.

Gratis licenses (referencing $\mathbf{\$ 0}$ in the Total field) are free. Please retain this printable license for your reference. No payment is required. 


\section{Curriculum Vitae}


Adam Goodwill

3132 Health Sciences North

Morgantown, WV 26506

Phone: (304) 293-4199

Email: agoodwill@hsc.wvu.edu

\section{EDUCATION}

Undergraduate

- Clarion University of Pennsylvania 1998-2003

B.S. in Molecular Biology/Biotechnology, B.S. in Biology, Minor in Chemistry

\section{Graduate}

- West Virginia University 2006-2011

Integrated Biomedical Sciences PhD Program, Physiology \& Pharmacology PhD Program

\section{COURSEWORK}

Undergraduate

- Analytical Chemistry/lab

- Anatomy and Physiology I/lab

- Applications of Microcomputers

- Biochemistry/lab

- Calculus and Analytical Geometry

- Cell Biology/lab

- Cell Physiology/lab

- Chemical Information \& Safety

- Computer Information Processing

- Elementary \& Applied Statistics

- General Microbiology/lab

\section{Graduate}

- Biostatistics

- Cardiovascular and Respiratory Biology

- Cell Structure and Metabolism

- Current Topics in Biochemistry

- Fundamentals of Integrated Systems

- Grad Physiology/Pharmacology 1

- Graduate Colloquium
- Genetics/lab

- Immunology/Lab

- Inorganic Chemistry/lab

- Mammalian Cell and Tissue Culture/lab

- Microbial Genetics/lab

- Molecular Biology/lab

- Organic Chemistry I \&II/labs

- Pathogenic Microbiology/lab

- Physical Chemistry/lab

- Undergraduate Seminar in Biology I \& II

- Vertebrate Embryology/Lab

- Immunology II

- Microbial Pathogenesis

- Molecular Genetics

- Physiology Literature

- Proteins and Proteomics

- Physiology of Inflammation

- Scientific Writing 


\section{RELEVANT EMPLOYMENT}

April 2006-August 2006 Research Assistant I, Jefferson Frisbee's Lab, W.V.U. In this position I served as one of two laboratory technicians in a lab studying the effects of obesity on the microcirculation. Specifically, I performed the experiments deemed to be molecular. These experiments, by and large, consisted of western blots, gelatin zymography and operation of the Luminex 200 system. Additionally, I aided in interpreting the data generated by the Luminex system.

July 2004-April $2006 \quad$ Research Assistant I, Thomas Elliott's Lab, W.V.U. In this position I served as the laboratory manager and sole research assistant for a lab studying the role of a specific stress factor, RpoS, in the gene regulation of the organism Salmonella typhimurium. During my time there, I was in charge of all ordering and record keeping. Additionally I generated several databases to search our extensive collection of custom oligos and strains. I also conducted experiments for the lab utilizing, primarily, my molecular biology and cell biology experience.

\section{BRIEF OVERVIEW OF CURRENT RESEARCH}

My work focuses on changes to both vascular network structure and vasomotor responses as a result of attainment and maintenance of a profoundly obese state. This state of obesity, along with the accompanying constellation of comorbidities (atherogenic dyslipidemia, prothrombotic environment, pro-oxidant environment, hypertrigylceridemia, hyperlipidemia, insulin resistance, hypertension, etc.) causes complex changes to the vasculature of afflicted animals and individuals. My current research tries to define the relationships between changes to the inflammatory profile, oxidant environment, vascular metabolism of arachidonic acid \& the ultimate consequent alterations to structure, function and perfusion-control within peripheral vascular networks.

\section{BOOK CHAPTERS AND REVIEWS}

1. P.A. Stapleton, A.G. Goodwill, M.E. James, R.W. Brock and J.C. Frisbee. "Interventional Strategies In Hypercholesterolemia" The Journal of Inflammation (Lond). 2010 Nov 18;7(1):54. [Epub ahead of print] PMID: 21087503

2. A.G. Goodwill and J.C. Frisbee. "Oxidant Stress and Skeletal Muscle Microvasculopathy in the Metabolic Syndrome" Humana Press/Springer Science Inc.; for "Oxidative Stress in Applied Basic Science and Clinical Research" [In Preparation].

3. P.A. Stapleton, M.E. James, A.G. Goodwill, J.C. Frisbee. Obesity and Vascular Dysfunction. Pathophysiology. 2008 Aug;15(2):79-89. [Epub 2008 Jun 20]. 


\section{PEER REVIEWED PUBLICATIONS}

1. J.T. Butcher, A.G. Goodwill, J.C. Frisbee. The Ex-Vivo Isolated Microvessel Preparation as a Method of Investigation into Skeletal Muscle Perfusion. The Journal of Visualized Experiments. [In Press]

2. J.C. Frisbee, F. Wu, A.G. Goodwill, J.T. Butcher, and D.A. Beard. Spatial Heterogeneity in Skeletal Muscle Microvascular Perfusion Distribution is Increased in the Metabolic Syndrome. Am J Physiol Regul Integr Comp Physiol 2011 Oct;301(4):R975-86. Epub 2011 Jul 20. PMID: 21775645

3. J.C. Frisbee, A.G. Goodwill, J.T. Butcher, and I.M. Olfert. Divergence Between Arterial Perfusion and Fatigue Resistance in Skeletal Muscle in the Metabolic Syndrome. Exp Physiol. 2011 Mar;96(3):369-83. Epub 2010 Dec 1. PMID:21123363

4. S.J. Frisbee, C.B. Chambers, J.C. Frisbee, A.G. Goodwill, and R.J. Crout. SelfReported Dental Hygiene, Obesity, and Systemic Inflammation in a Pediatric Rural Community Cohort. BMC Oral Health 2010 Sep 18;10(1):21. PMID: 20849640

5. J. C. Frisbee, A.G. Goodwill, P.A Stapleton, S.J. Frisbee, and A.C. d'Audiffret. Aspirin Resistance with Genetic Dyslipidemia: Contribution of Vascular Thromboxane Generation. Physiologic Genomics. 2010 Jun 8. [Epub ahead of print]

PMID: 20530721

6. S.J. Frisbee, C.B. Chambers, J.C. Frisbee, A.G. Goodwill and R.J. Crout. Association Between Dental Hygiene, Cardiovascular Disease Risk, and Systemic Inflammation in Rural Adults. J Dent Hyg. 2010;84(4):177-84. [Epub 2010 Nov 1]. PMID: 21047463

7. A.C. d'Audiffret, S.J. Frisbee, P.A. Stapleton, A.G. Goodwill, M.E. James, E. Isingrini and J.C. Frisbee. Depressive Behavior and Vascular Dysfunction: A Link Between Clinical Depression AND Vascular Disease?. The Journal of Applied Physiology. 2010 Feb 18. [Epub ahead of print]. PMID: 20167667

8. T.R. Nurkiewicz, D.W. Porter, A.F. Hubbs, S. Stone, A.M. Moseley, J.L. Cumpston, A.G. Goodwill, S.J. Frisbee, P.L. Perrotta, R.W. Brock, J.C. Frisbee, M.A. Boegehold, D.G. Frazer, B.T. Chen and V. Castranova. Pulmonary Particulate Matter and Systemic Microvascular Dysfunction. Health Effects Institute [In Review], 2009.

9. A.G. Goodwill, S.J. Frisbee, P.A. Stapleton, M.E. James and J.C. Frisbee. Impact of Chronic Anti-Cholesterol Therapy on Development of Microvascular Rarefaction in the Metabolic Syndrome. Microcirculation. 2009 Nov;16(8):66784. PMID: 19905967

10. J.B. Samora, A.G. Goodwill, J.C. Frisbee, M.A. Boegehold. Growth-Dependant Changes in the Contribution of Carbon Monoxide to Artertiolar Function. Journal of Vascular Research. 2010;47(1):23-34. [Epub 2009 Aug 6]. PMID: 19672105

11. A.G. Goodwill, P.A. Stapleton, M.E. James, J.C. Frisbee. Increased Arachidonic Acid-Induced Thromboxane Generation Impairs Skeletal Muscle Arteriolar Dilation with Genetic Dyslipidemia. Microcirculation. 2008 Oct;15(7):621-31. PMID: 18720229 
12. A.G. Goodwill, M.E. James, J.C. Frisbee. Increased vascular thromboxane generation impairs dilation of skeletal muscle arterioles of obese Zucker rats with reduced oxygen tension. Am J Physiol Heart Circ Physiol. 2008 Oct;295(4):H1522-8. Epub 2008 Aug 8. PMID: 18689495

13. P.A. Stapleton, A.G. Goodwill, M.E. James, J.C. Frisbee. Altered Mechanisms of Endothelium-Dependent Dilation in Skeletal Muscle Arterioles with Genetic Hypercholesterolemia. Am J Physiol Regul Integr Comp Physiol. 2007 Sep;293(3):R1110-9. PMID: 17626122

14. Amy M. Jones, Adam Goodwill and T. Elliott. Limited Role for the DsrA and RprA Regulatory RNAs in rpoS Regulation in Salmonella enterica. J Bacteriol. 2006 Jul;188(14):5077-88. PMID: 16816180

\section{CONFERENCE PRESENTATIONS}

1. Invited Lecture: Tumor necrosis factor alpha contributes to vascular dysfunction and skeletal muscle microvascular rarefaction in the obese Zucker rat. A.G. Goodwill, J.T. Butcher, J.C. Frisbee. Experimental Biology 2011/Microcirculatory Society President's Symposium II, Washington D.C., District of Columbia, United States, 2011-04; Abstract 636.16

2. Poster: Temporal progression of microvascular rarefaction in the metabolic syndrome. A.G. Goodwill, J.C. Frisbee. Experimental Biology 2011, Washington D.C., District of Columbia, United States, 2011-04; Abstract 636.17

3. Poster: Differential impact of myogenic activation on dilator responses in cerebral and skeletal muscle resistance arterioles of obese Zucker rats. J.T. Butcher, A.G. Goodwill, J.C. Frisbee. Experimental Biology 2011, Washington D.C., District of Columbia, United States, 2011-04; Abstract 816.9

4. Poster: Divergence between arterial perfusion and fatigue resistance in skeletal muscle in the metabolic syndrome. J. C. Frisbee, A.G. Goodwill, J.T. Butcher, I.M. Olfert. Experimental Biology 2011, Washington D.C., District of Columbia, United States, 2011-04; Abstract 1023.7

5. Poster: Effect of remote ischemic preconditioning on hepatic parenchymal and microvascular damage in obesity. H.A. O'Leary, A. Kothur, S.B. Fournier, A.G. Goodwill, J.C. Frisbee, R.W. Brock. Experimental Biology 2011, Washington D.C., District of Columbia, United States, 2011-04; Abstract 1117.9

6. Poster: Alterations in hepatic tumor cell colonization during obesity. H.A. O'Leary, A. Kothur, A.G. Goodwill, J.C. Frisbee, R.W. Brock. Experimental Biology 2011, Washington D.C., District of Columbia, United States, 2011-04; Abstract 639.2

7. Poster: Tumor necrosis factor alpha contributes to vascular dysfunction and skeletal muscle microvascular rarefaction in the obese Zucker rat. A.G. Goodwill, J.T. Butcher, J.C. Frisbee. Experimental Biology 2011, Washington D.C., District of Columbia, United States, 2011-04; Abstract 636.16 
8. Poster: Obesity-induced increased vascular thromboxane $A_{2}$ Generation: An initiating stimulus for microvascular rarefaction? A.G. Goodwill, P.A. Stapleton, S.J. Frisbee, and J.C. Frisbee. World Congress for Microcirculation 2010, Paris France. 2010-09

9. Poster: Aspirin resistance with genetic dyslipidemia: Contribution of vascular thromboxane generation. J.C. Frisbee, A.G. Goodwill, P.A. Stapleton, S.J. Frisbee, and A.C. d'Audiffret. World Congress for Microcirculation 2010, Paris, France. 2010-09

10. Poster: Increased vascular generation of thromboxane $A_{2}$ : An initiating condition for microvascular rarefaction in obese Zucker rats? A.G. Goodwill, P.A. Stapleton, S.J. Frisbee, M.E. James and J.C. Frisbee. Experimental Biology 2010, Anaheim, California, United States. 2010-04; Abstract 774.19

11. Poster: Insulin resistance-independent impairments to arterial endothelial function with depressive symptoms in mice. J.A.L. Beckett, M.E. James, P.A. Stapleton, A.G. Goodwill, A.C. d'Audiffret and J.C. Frisbee. Experimental Biology 2010, Anaheim, California, United States. 2010-04; Abstract 1044.3

12. Poster: Impaired arteriolar dilation in a mouse model of familial hypercholesterolemia and anti-cholesterol therapy. P.A. Stapleton, A.G. Goodwill, M.R. Morrisette, M.E. James and J.C. Frisbee. Experimental Biology 2010, Anaheim, California, United States. 2010-04; Abstract 593.5

13. Invited Lecture: Correlations between peripheral vascular function, inflammation and depression in human subjects. P.A. Stapleton, A.C. d'Audiffret, S.J. Frisbee, A.G. Goodwill, M.E. James, and J.C. Frisbee. Experimental Biology 2009, New Orleans, Louisiana, United States. 2009-04; Abstract 23:795.3.

14. Poster: Vascular thromboxane generation restrains arteriolar hypoxic dilation in skeletal muscle of OZRs. A.G. Goodwill, J.M. Hollander, R.W. Brock, M.E. James and J.C. Frisbee. Experimental Biology 2009, New Orleans, Louisiana, United States. 2009-04; Abstract 23:767.9.

15. Poster: AA-induced TXA2 generation impairs skeletal muscle arteriolar dilation with genetic dyslipidemia. A.G. Goodwill, P.A. Stapleton, M.E. James, A.C. d'Audiffret and J.C. Frisbee. Experimental Biology 2009, New Orleans, Louisiana, United States. 2009-04; Abstract 23:766.3.

16. Poster: Development of a predictive model for negative microvascular outcomes in the metabolic syndrome. J.C. Frisbee, A.G. Goodwill, M.E. James, R.W. Brock, J.M. Hollander and S.J. Frisbee. Experimental Biology 2009, New Orleans, Louisiana, United States. 2009-04; Abstract 23:594.11.

17. Poster: Mechanistic links between the lung and the systemic microcirculation after nanoparticle exposure. T.R. Nurkiewicz, M. Donlin, A.F. Hubbs, A.G. Goodwill, J.C. Frisbee, B.T. Chen, D.G. Frazer and V. Castranova. Society of 
Toxicology, Baltimore, Maryland, United States. Toxicological Sciences, March 108 (1-S), 1353, 2009.

18. Poster: Diesel exhaust exposure alters microvascular blood flow and wall shear rate. K.L. Sites, A.G. Goodwill, J.C. Frisbee and T.R. Nurkiewicz. Society of Toxicology, Baltimore, Maryland, United States. March 108 (1-S), 200, 2009.

19. Invited Lecture: Metabolic Syndrome and Microvascular Rarefaction: Contributions from Nitric Oxide and Inflammation. J.C. Frisbee, A.G. Goodwill, P.A. Stapleton, M.E. James, R.W. Bryner, and S.J. Frisbee. The 25th Conference of the European Society for Microcirculation; Integrating Vascular Biology and Medicine, Budapest, Hungary. 2008-08

20. Invited Lecture: Microvascular Adaptations to Obesity and the Metabolic Syndrome. J. Frisbee, A.G. Goodwill, M. James, R. Bryner, S. Frisbee. 2008 APS Intersociety Meeting: The Integrative Biology of Exercise V, Hilton Head, South Carolina, United States. 2008-08

21. Invited Lecture: The Metabolic Syndrome and Microvascular Rarefaction: Integrated Model for NO Bioavailability. J.C. Frisbee, A.G. Goodwill, P.A. Stapleton, M.E. James, R.W. Bryner, S.J. Frisbee European Society for Microcirculation Annual Meeting 2008, Budapest, Hungary. 2008-08

22. Poster: Dental Habits Related to Adult Cardiovascular Disease and Systemic Inflammation. S.J. Frisbee, C.B. Chambers, J.C. Frisbee, A.G. Goodwill, R.J. Crout. The International Association for Dental Research (IADR) 37th Annual Meeting \& Exhibition, Dallas, Texas, United States. 2008-04

23. Poster: Dental health, cardiovascular disease risk and systemic inflammation in children. C.B. Chambers, S.J. Frisbee, J.C. Frisbee, A.G. Goodwill and R.J. Crout. The International Association for Dental Research (IADR) 37th Annual Meeting \& Exhibition, Dallas, Texas, United States. 2008-04

24. Poster: Arachidonic acid-induced vascular thromboxane generation in mouse models of atherothrombotic vascular disease. J.C. Frisbee, M.E. James, A.G. Goodwill and C. Khamare. 2007-05

25. Poster: Influences of age and gender on cardiovascular disease risk factor associated inflammation. S.J. Frisbee, A.G. Goodwill, J.M. Delp and J.C. Frisbee. American Heart Association, Epidemiology Council Meeting, 2007, Orlando, Florida, United States. 2007-02

26. Poster: Altered Patterns of Inflammatory Marker Expression in Plasma of Obese vs. Dyslipidemic Humans. S.J. Frisbee, A.G. Goodwill, M. Boegehold, W.A. Neal, J.C. Frisbee. North American Association for the study of obesity (NAASO) Annual Scientific Meeting, Boston, Massachusetts, United States. 2006-10

27. Invited Lecture: Inflammation, NO bioavailability and the metabolic syndrome: interactions leading to rarefaction. J.C. Frisbee, A.G. Goodwill and S.J. Frisbee. Workshop on Mathematical Modeling of Microcirculation; Mathematical 
Biosciences Institute at Ohio State University, Columbus, Ohio, United States. 2006-10

28. Poster: Low Vascular NO Bioavailability-Induced Microvascular Rarefaction is not Associated with Angiostatin. J.C. Frisbee, M.E. James, J. Balch Samora, A.G. Goodwill, B. Chelladurai and D.P. Basile. Arteriosclerosis, Thrombosis and Vascular Biology 2006 Scientific Sessions, Washington D.C., District of Columbia, United States. 2006-04

29. Lecture: Regulation of RpoS in enteric bacteria. Hirsch, M., A.M. Jones, A. Goodwill \& T. Elliott. The Seventh Analytical Genetics Meeting, San Diego, California, United States. 2005-10

30. Lecture: Production of Green Sheen Differs Depending Upon Atmospheric Conditions and Medium Formulations. Adam G. Goodwill, Heather A. O'Leary, Amanda M. Hartle, Kate H. Eggleton, and Douglas M. Smith. 2003-04

31. Poster: Identification of the Green Sheen Gene In Uropathogenic E. coli. Essandoh, Eugene, Leonard Kotevski, Heather O'Leary, Adam Goodwill, Carrie Martz, Josh Yeykal, Rebhecca Parisi, Kate Eggleton, Douglas M. Smith. Commonwealth of Pennsylvania University Biologists 34th Annual Meeting, Shippensburg, Pennsylvania, United States. 2003-04

32. Poster: Production of Green Sheen Differs Depending on Atmospheric Conditions and EMB Medium Formulations. Adam G. Goodwill, Heather A. O'Leary, Douglas M. Smith, Ph.D. 2002 Annual meeting of the Allegheny Branch of the American Society for Microbiology, Clarion, Pennsylvania, United States. 2002-10

33. Poster: Production Of Green Sheen Differs Depending Upon Atmospheric Conditions and Medium Formulations. Goodwill, A.G., H. A. O'Leary, A. M. Hartle, K. H. Eggleton and D. M. Smith. Commonwealth of Pennsylvania University Biologists 33rd Annual Meeting, Lock Haven, Pennsylvania, United States. 2002-04

\section{INSTITUTIONAL PRESENTATIONS}

1. Lecture: Microvascular Rarefaction in the Metabolic Syndrome: Goodwill A.G. The Department of Physiology \& Pharmacology Julie Betschard Symposium 2011-05

2. Invited Lecture: Temporal Progression of Microvascular Rarefaction in the Metabolic Syndrome: Goodwill A.G. The West Virginia University 2011 E.J. Van Liere Memorial Convocation and Research Day. 2011-03

3. Lecture: Temporal Progression of Microvascular Rarefaction in the Metabolic Syndrome: Goodwill A.G. The Department of Physiology and Pharmacology required annual seminar. 2011-02. 
4. Invited Lecture: Microvascular Rarefaction in the Obese Zucker Rat: Goodwill A.G. The West Virginia University School of Pharmacy Research Day. 2010-10.

5. Lecture: Increased Vascular Generation of Thromboxane A2: An Initiating Condition For Microvascular Rarefaction in Obese Zucker Rats? Goodwill A.G. The Department of Physiology \& Pharmacology Julie Betschard Symposium. 2010-05

6. Lecture: Aspirin Resistance with Genetic Dyslipidemia: Contribution of Vascular Thromboxane Generation. Goodwill A.G. The Department of Physiology and Pharmacology required annual seminar. 2010-04

7. Lecture: Vascular Consequences of Obesity Induced Alterations to Arachidonic Acid Metabolism in Obese Zucker Rats. Goodwill A.G. The West Virginia University Center for Cardiovascular and Respiratory Sciences monthly work in progress meeting. 2009-12

8. Lecture: Impact of Chronic Anti-Cholesterol Therapy on Development of Microvascular Rarefaction in the Metabolic Syndrome. Goodwill A.G. The Department of Physiology and Pharmacology required annual seminar. 2009-09

9. Lecture: Increased Vascular Thromboxane Generation Restrains Arteriolar Dilation to Hypoxia in Obese Zucker Rats. Goodwill A.G. The Department of Physiology \& Pharmacology Julie Betschard Symposium. 2009-05

10. Poster: Elevated Vascular Thromboxane Generation Impairs Dilation of OZR Arterioles with Reduced $\mathrm{O}_{2}$ Tension. Goodwill A.G. The West Virginia University 2009 E.J. Van Liere Memorial Convocation and Research Day.2009-04

11. Lecture: Arachidonic Acid-Induced TXA2 Generation Impairs Arteriolar Dilation with Genetic Dyslipidemia. Goodwill A.G.. The Department of Physiology \& Pharmacology Julie Betschard Symposium. 2008-05

12. Lecture: Increased Arachidonic Acid-Induced Thromboxane Generation Impairs Skeletal Muscle Arteriolar Dilation with Genetic Dyslipidemia. Goodwill A.G. The Department of Physiology and Pharmacology required annual seminar. 2008-04

\section{TEACHING EXPERIENCE}

- Special Topics in Physiology; West Virginia University 2009

- I facilitated a study group in which specific topics including cardiovascular, respiratory, and renal physiology were expanded from the perspective of clinical relevance. This course, designed for medical students, sought to integrate clinical case studies with the basic biomedical sciences required to understand the processes of the pathologies presented within those studies. This course was facilitated by 3 separate instructors. 
- Cardiovascular Physiology Study Session; West Virginia University 2008-2009

- Facilitated a 3 to 5 hour per week study group to aid select pharmacy professional students in increasing their understanding of cardiovascular physiology. I was asked to facilitate this tutoring group by the Pharmacy department. This was only offered during the cardiovascular modules of their normal coursework each year.

- Genetics Study Session; Clarion University

2002-2003

- Co-taught a supplementary course designed to complement didactic materials for the genetics course which was required for all undergraduates in a biology discipline at Clarion University. This course reinforced concepts taught within the undergraduate genetics curriculum.

\section{INSTITUTIONAL SERVICE}

1. Graduate Student Representative, West Virginia University Vice President for Research and Development Search Committee; 2011

2. Student Member, West Virginia University Vice President for Health Sciences Research and Graduate Education Search Committee; 2010

\section{SERVICE TO SCIENTIFIC SOCIETIES}

1. Microcirculatory Society Young Investigator Symposium Co-Chair. Experimental Biology, Washington D.C., District of Columbia, United States. $\quad$ 2011-04

\section{AWARDS \& FELLOWSHIPS}

1. Runner up, Best Oral Presentation, Julie Betschart Symposium W.V.U. Dept of Physiology and Pharmacology

2011

2. Runner up, American Society for Pharmacology and Experimental Therapeutics Cardiovascular Section Graduate Student Best Abstract Award

3. Society for Experimental Biology and Medicine, Burton E. Sobel Award for Excellence in Cardiovascular Research

2011

4. American Society for Pharmacology and Experimental Therapeutics Graduate Student Travel Award; Experimental Biology

5. Runner up, Best Oral Presentation The West Virginia University E.J. Van Liere Memorial Convocation/

6. Best Oral Presentation, Julie Betschart Symposium W.V.U. Dept of Physiology and Pharmacology 
7. ORAU-Fellowship for $60^{\text {th }}$ Annual Lindau Meeting of Nobel Laureates in Chemistry, Physics, and Physiology/Medicine Lindau, Germany

8. American Heart Association - Great Rivers Affiliate "Early Microvascular Rarefaction in Metabolic Syndrome" 10PRE3040016

2010-2012

9. Research Training in Cardiovascular and Pulmonary Diseases (Predoctoral Fellowship) 5T32HL090610-02.

2009-2010

10. Best Oral Presentation, Julie Betschart Symposium W.V.U. Dept of Physiology and Pharmacology

11. Zweifach Student Travel Award, Microcirculatory Society

2009

12. Best Oral Presentation CPUB

13. Best Poster Presentation CPUB

2009

2003

14. Runner up, Best Poster Presentation ABASM

2002

2002

\section{PROFESSIONAL SOCIETY MEMBERSHIPS}

1. Society for Experimental Biology \& Medicine, 2011 (Student Member)

2. American Physiological Society, 2011 (Student Member)

3. American Society for Pharmacology and Experimental Therapeutics, 2011 (Student Member)

4. Microcirculatory Society, USA 2009-2011 (Student Member)

5. American Society for Microbiology 2001-2003 (Student Member) 